\title{
A INFLUÊNCIA DA IDADE E DA REPOSIÇÃO \\ HORMONAL SOBRE A MODULAÇÃO AUTONÔMICA DO CORAÇÃO E O LIMIAR DE ANAEROBIOSE
}

Valéria Ferreira

Dissertação apresentada ao Programa de Pós-Graduação Interunidades em Bioengenharia - Escola de Engenharia de São Carlos / Faculdade de Medicina de Ribeirão Preto / Instituto de Química de São Carlos da Universidade de São Paulo, para obtenção do título de Mestre em Bioengenharia.

ORIENTADORA: Profa. Dra. Ester da Silva

São Carlos 
Investigação conduzida no Núcleo de Pesquisa em Exercício Físico do Laboratório de Fisioterapia Cardiovascular do Departamento de Fisioterapia da Universidade Federal de São Carlos e no Laboratório de Fisiologia do Exercício da Divisão de Cardiologia do Departamento de Clínica Médica do Hospital das Clínicas da Faculdade de Medicina de Ribeirão Preto da Universidade de São Paulo. Apoio Financeiro: CAPES, FAPESP e CNPq. 
"Mais importante que a busca pela certeza é a busca pela clareza".

François Gautier, filósofo 


\section{Dedicatória}

Aos meus pais, Ariovaldo e Terezinha "in memorian", que muitas vezes renunciaram seus sonhos para realizarem os meus.

Às minhas irmãs, Ana Paula "in memorian" e Teca, por serem pessoas tão maravilhosas e exemplos de superação.

Às minhas avós, Ermelinda e Rosa "in memorian", pelo apoio e incentivo em todos os momentos da minha vida.

Ao meu noivo, Marcelo, pelo privilégio de ter você ao meu lado, sempre carinhoso, compreensivo, amoroso... 
À Profa. Dra. Ester da Silva, minha orientadora e amiga, pelo apoio, sugestões e críticas que muito contribuíram para minha formação científica e para o desenvolvimento deste trabalho. 
Agradecimento especial

A todas voluntárias, por terem cedido algumas horas de suas vidas para que eu pudesse realizar este trabalho.

A todas vocês, meu respeito e gratidão. 


\section{Agradecimentos}

- A Deus, sempre presente em minha vida, iluminando o meu caminho e dando-me força e serenidade para superar os obstáculos.

- À minha família, pelo carinho e compreensão das ausências destes anos.

- Ao Prof. Dr. Lourenço Gallo Júnior, por ter me dado a oportunidade de realizar os experimentos em seu laboratório e partilhar comigo os seus conhecimentos.

- À Profa. Dra. Aparecida Maria Catai, pela disponibilidade, críticas e contribuições valiosas no transcorrer deste trabalho, que com certeza foram de extrema relevância para 0 enriquecimento desta dissertação.

- Ao Prof. Dr. José Carlos Pereira, pelas críticas e sugestões durante o exame de qualificação, que contribuíram para o aprimoramento deste trabalho.

- Ao Prof. Dr. Marcos Felipe Silva de Sá, pelo apoio e incentivo para a realização deste trabalho.

- À amiga Tatiane Flores Ribeiro, pela amizade e por ter partilhado comigo seus dados.

- Ao Prof. Dr. George Dantas, por suas sugestões e pela ajuda indispensável na triagem das voluntárias.

- A todos os funcionários do HCFMRP-USP que de alguma forma contribuíram para esta pesquisa. 
- Ao Prof. Dr. Jorge Oishi, por ter acreditado em mim e me incentivado para que seguisse os caminhos da pesquisa científica.

- À amiga Patrícia Driusso, por sua amizade e pela paciência em me ouvir em momentos difíceis da minha vida.

- A todos os amigos do Laboratório de Fisiologia do Exercício do Hospital das Clínicas da Faculdade de Medicina de Ribeirão Preto USP: Júlio, Renata, Ana Cláudia, Michele, Cleide e Valéria Papa, pela amizade, apoio e disponibilidade em ajudar na realização dos experimentos deste trabalho.

- A todos os amigos do Laboratório de Fisioterapia Cardiovascular da Universidade Federal de São Carlos: Lílian, Ana Paula, Robison, Luciana, Fabrício, Daniel, Albaíza, Vera, Fernanda, Cristiano, Karla, Pozzi, Lucas, Liliana, pela convivência, amizade e apoio irrestritos.

- Ao amigo Lucien, pela amizade e pela paciência e disponibilidade em ensinar a utilizar os aplicativos de aquisição e análise dos dados.

- Aos amigos da Bioengenharia, especialmente à Carla, à Angélica e à Elizete, pela amizade e carinho.

- Aos professores do Programa de Pós-Graduação em Bioengenharia, em especial ao professor Orivaldo Lopes da Silva, pela colaboração, apoio e amizade.

- À secretária Janete da Bioengenharia, pela atenção, carinho e presteza nos atendimentos.

- Aos órgãos de fomento CAPES, FAPESP e CNPq, pelo apoio financeiro que possibilitou a realização deste trabalho. 


\section{SUMÁRIO}

LISTA DE FIGURAS

i

LISTA DE TABELAS

LISTA DE ABREVIATURAS E SIGLAS ............................................

RESUMO

ABSTRACT

1. INTRODUÇÃO

2. OBJETIVO

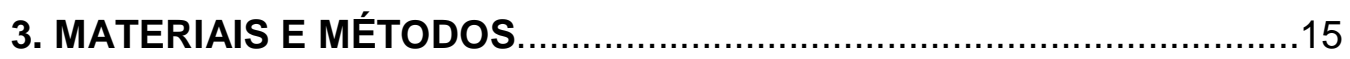

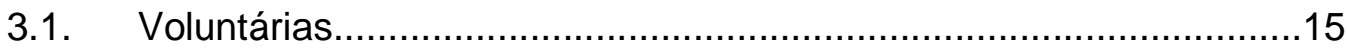

3.2. Critérios de inclusão das voluntárias............................................16

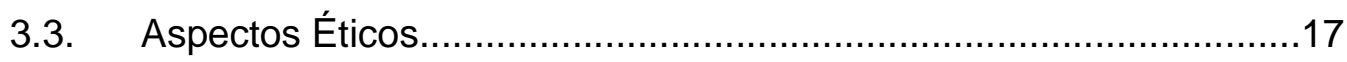

3.4. Avaliação Clínica.................................................................

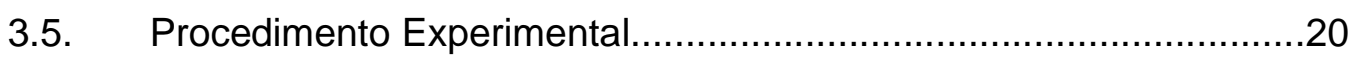

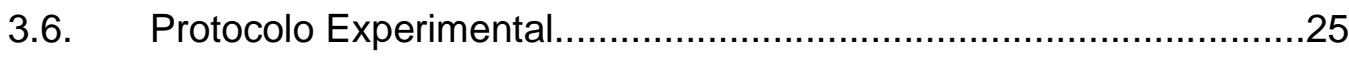

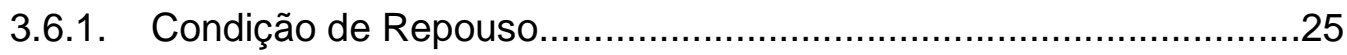

3.6.2. Teste de esforço físico dinâmico descontínuo do tipo degrau

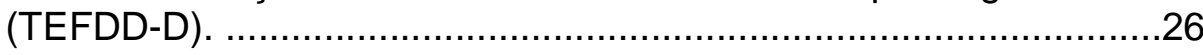

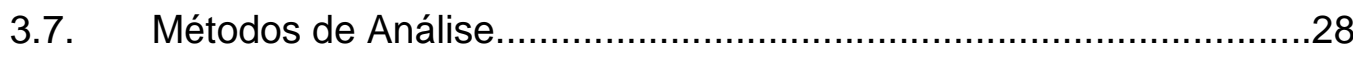

3.7.1. Análise da resposta da freqüência cardíaca nas condições de repouso e durante o teste de esforço físico dinâmico descontínuo do tipo degrau...

3.7.2. Variação da freqüência cardíaca e tempo de duração desta variação no início do exercício físico dinâmico descontínuo...

3.7.3. Variabilidade da freqüência cardíaca nas condições de repouso e durante o teste de esforço físico dinâmico descontínuo do tipo degrau.

3.7.4. Análise da resposta da freqüência cardíaca na determinação do limiar de anaerobiose por meio do modelo matemático e estatístico semiparamétrico. 
3.7.5. Análise da variabilidade da freqüência cardíaca na determinação do limiar de anaerobiose por meio dos índices de RMSSD dos intervalos R-R.

3.8. Metodologia Estatística..............................................................

4. RESULTADOS .40

4.1. Idade, características antropométricas e clínicas das voluntárias estudadas

4.2. Análise descritiva da freqüência cardíaca e de sua variabilidade.

4.3. Análise da freqüência cardíaca e de sua variabilidade durante o repouso.

4.4. Análise da variação da freqüência cardíaca e do tempo na fase inicial do exercício físico.

4.5. Análise da resposta da freqüência cardíaca e de sua variabilidade durante o teste de esforço físico dinâmico descontínuo do tipo degrau.

4.6. Análise da determinação do limiar de anaerobiose pelo modelo matemático e estatístico semiparamétrico, e pelo índice de RMSSD dos intervalos R-R.

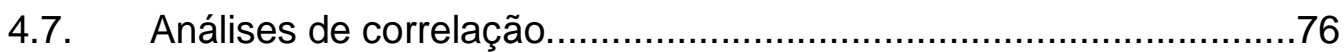

4.7.1. Análise de correlação entre os índices de RMSSD dos intervalos $\mathrm{R}-\mathrm{R}$ e as potências do teste de esforço físico dinâmico descontínuo do tipo degrau.

4.7.2. Análise de correlação entre os índices de RMSSD dos intervalos R-R nos níveis de potência do limiar de anaerobiose determinado pelo modelo matemático e estatístico semiparamétrico e pela análise estatística dos índices de RMSSD

\section{DISCUSSÃO}

5.1. Características das voluntárias

5.2. Análise da resposta da freqüência cardíaca e de sua variabilidade durante $\mathrm{o}$ repouso nas posições supina $e$ sentada.

5.3. Análise da resposta da freqüência cardíaca e de sua variabilidade ao exercício físico dinâmico

5.4. Identificação do limiar de anaerobiose pelo modelo matemático e estatístico semiparamétrico e pela variabilidade da freqüência cardíaca. 
6. CONCLUSÃO

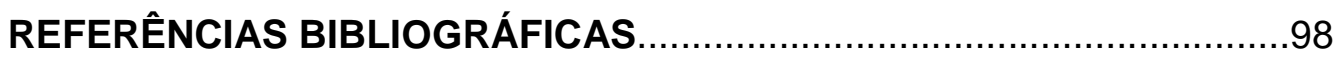

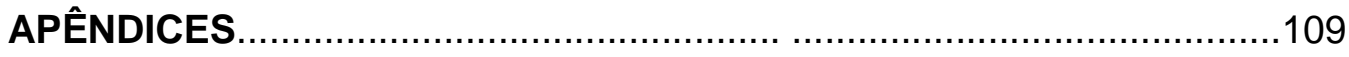

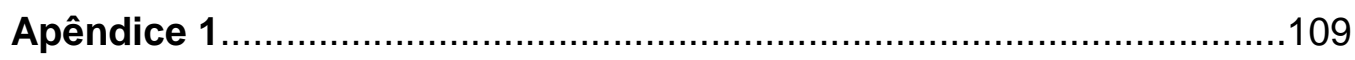

Apêndice 2

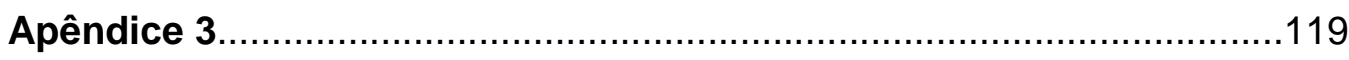




\section{LISTA DE FIGURAS}

FIGURA 1. Representação esquemática do teste de esforço físico dinâmico contínuo, realizado em esteira elétrica automática com protocolo de Bruce modificado II

FIGURA 2. Ilustração dos equipamentos utilizados durante o teste de esforço físico dinâmico descontínuo do tipo degrau realizado no Laboratório de Fisiologia do Exercício da Divisão de Cardiologia do Hospital das Clínicas da Faculdade de Medicina de Ribeirão Preto da USP. Em A: bicicleta ergométrica de frenagem eletromagnética. Em B: microcomputador com sistema de captação de sinal de eletrocardiograma.

FIGURA 3. Ilustração do procedimento experimental durante o teste de esforço físico dinâmico descontínuo do tipo degrau realizado no Núcleo de Pesquisa em Exercício Físico (NUPEF) do Laboratório de Fisioterapia Cardiovascular da UFSCar

FIGURA 4. Ilustração da tela do computador do programa de aquisição do eletrocardiograma e da freqüência cardíaca, batimento a batimento, em tempo real, durante o repouso na posição sentada de uma das voluntárias jovens estudadas (ACM)

FIGURA 5. Representação esquemática do protocolo do teste de esforço físico dinâmico descontínuo do tipo degrau, com incrementos de potência progressiva de 5 em 5 Watts com duração de 240 segundos em cada nível de esforço, com períodos de repouso intercalados.

FIGURA 6. llustração da tela do computador do programa "Analisador gráfico de intervalos R-R, freqüência cardíaca e eletrocardiograma", contendo informações do protocolo utilizado, planilha de dados absolutos de freqüência cardíaca, batimento a batimento, intervalos $\mathrm{R}$-R e tempos correspondentes, gráficos das respostas da freqüência cardíaca em repouso e em exercício físico dinâmico 
FIGURA 7. Resposta da freqüência cardíaca, em bpm, obtida batimento a batimento, em tempo real, durante 60 segundos em repouso, 240 segundos em exercício físico na potência de 35 Watts e 60 segundos em recuperação, de uma das voluntárias jovens estudadas (RTK)

FIGURA 8. Ajuste do modelo matemático e estatístico semiparamétrico ao conjunto de dados de freqüência cardíaca no período de 120 a 300 segundos de exercício físico, mostrando tendências positivas de inclinação da reta, na potência de 35 Watts do teste de esforço físico dinâmico descontínuo do tipo degrau, realizado por uma das voluntárias jovens estudadas (RTK)

FIGURA 9. Relatório da análise do ajuste do modelo matemático e estatístico semiparamétrico, aos dados de freqüência cardíaca obtidos durante teste de esforço físico dinâmico descontínuo do tipo degrau na potência de 35 Watts, de uma das voluntárias jovens estudadas (RTK)

FIGURA 10. Gráfico de probabilidade normal dos resíduos da série de dados de freqüência cardíaca ajustados pelo modelo matemático e estatístico semiparamétrico, no período de 120 a 300 segundos de exercício físico, na potência de 35 Watts do teste de esforço físico dinâmico descontínuo do tipo degrau, de uma das voluntárias jovens estudadas (RTK). .36

FIGURA 11. Análise dos valores absolutos dos intervalos R-R para a potência de 45 Watts de uma das voluntárias pósmenopausa com reposição hormonal estudada quanto à distribuição dos dados. Em A, estão representados os valores observados em relação aos esperados e em B, estão representados a Curva de Gauss e o Histograma do número de observações.

FIGURA 12. Resposta da freqüência cardíaca em batimentos por minuto (bpm), batimento a batimento, em tempo real, durante 360 segundos (s) em repouso, nas posições supina e sentada e durante $60 \mathrm{~s}$ em repouso préesforço, $240 \mathrm{~s}$ de esforço físico dinâmico com potências progressivas de 15, 20, 25, 30, 35, 40, 45 e 50 Watts (W) e $60 \mathrm{~s}$ após o esforço, de uma das voluntárias jovens estudada (CCESO) 
FIGURA 13. Resposta da freqüência cardíaca em batimentos por minuto (bpm), batimento a batimento, em tempo real, durante 360 segundos (s) em repouso, nas posições supina e sentada e durante $60 \mathrm{~s}$ em repouso préesforço, $240 \mathrm{~s}$ de esforço físico dinâmico com potências progressivas de $15,20,25,30,35,40$ e 45 Watts (W) e $60 \mathrm{~s}$ após o esforço, de uma das voluntárias pós-menopausa sem reposição hormonal estudada (DBS)

FIGURA 14. Resposta da freqüência cardíaca, em batimentos por minuto (bpm), batimento a batimento, em tempo real, durante 360 segundos (s) em repouso, nas posições supina e sentada e durante $60 \mathrm{~s}$ em repouso préesforço, $240 \mathrm{~s}$ de esforço físico dinâmico com potências progressivas de $15,20,25,30,35,40$ e 45 Watts (W) e $60 \mathrm{~s}$ após o esforço, de uma das voluntárias pós-menopausa com reposição hormonal estudada (ESV).

FIGURA 15. Valores de freqüência cardíaca, em batimentos por minuto (bpm), para o grupo de voluntárias jovens $(n=10)$, pós-menopausa sem reposição hormonal (PMSRH) $(\mathrm{n}=13)$ e pós-menopausa com reposição hormonal $(\mathrm{PMCRH})(\mathrm{n}=9)$, durante o repouso, nas posições supina e sentada. Nível de significância de $\alpha=0,05$

FIGURA 16. Valores dos índices de RMSSD dos intervalos R-R, em milissegundos (ms), para o grupo de voluntárias jovens $(n=10)$, pós-menopausa sem reposição hormonal (PMSRH) $(n=13)$ e pós-menopausa com reposição hormonal (PMCRH) $(n=9)$, durante 0 repouso, nas posições supina e sentada. Nível de significância de $\alpha=0,05$.

FIGURA 17. Em A, valores do tempo de variação da freqüência cardíaca, em segundos (s) e em B, valores de variação da freqüência cardíaca, em batimentos por minuto (bpm), na potência de 15 Watts do teste de esforço físico dinâmico descontínuo do tipo degrau, para o grupo de voluntárias jovens $(n=10)$, pósmenopausa sem reposição hormonal (PMSRH) $(n=13)$ e pós-menopausa com reposição hormonal (PMCRH) $(n=9)$. Nível de significância de $\alpha=0,05$ 
FIGURA 18. Em A, valores do tempo de variação da freqüência cardíaca, em segundos (s) e em B, valores de variação da freqüência cardíaca, em batimentos por minuto (bpm), na potência de 20 Watts do teste de esforço físico dinâmico descontínuo do tipo degrau, para o grupo de voluntárias jovens $(n=10)$, pósmenopausa sem reposição hormonal (PMSRH) $(n=13)$ e pós-menopausa com reposição hormonal $(P M C R H)(n=9)$. Nível de significância de $\alpha=0,05$.

FIGURA 19. Em A, valores do tempo de variação da freqüência cardíaca, em segundos (s) e em B, valores de variação da freqüência cardíaca, em batimentos por minuto (bpm), na potência de 25 Watts do teste de esforço físico dinâmico descontínuo do tipo degrau, para o grupo de voluntárias jovens $(n=10)$, pósmenopausa sem reposição hormonal (PMSRH) $(n=13)$ e pós-menopausa com reposição hormonal $(\mathrm{PMCRH})(\mathrm{n}=9)$. Nível de significância de $\alpha=0,05$.

FIGURA 20. Em A, valores do tempo de variação da freqüência cardíaca, em segundos (s) e em B, valores de variação da freqüência cardíaca, em batimentos por minuto (bpm), na potência de 30 Watts do teste de esforço físico dinâmico descontínuo do tipo degrau, para o grupo de voluntárias jovens $(n=10)$, pósmenopausa sem reposição hormonal (PMSRH) $(n=13)$ e pós-menopausa com reposição hormonal $(\mathrm{PMCRH})(\mathrm{n}=9)$. Nível de significância de $\alpha=0,05$

FIGURA 21. Em A, valores do tempo de variação da freqüência cardíaca, em segundos (s) e em B, valores de variação da freqüência cardíaca, em batimentos por minuto (bpm), na potência de 35 Watts do teste de esforço físico dinâmico descontínuo do tipo degrau, para o grupo de voluntárias jovens $(n=10)$, pósmenopausa sem reposição hormonal (PMSRH) $(n=13)$ e pós-menopausa com reposição hormonal $(\mathrm{PMCRH})(\mathrm{n}=9)$. Nível de significância de $\alpha=0,05$ 
FIGURA 22. Em A, valores do tempo de variação da freqüência cardíaca, em segundos (s) e em B, valores de variação da freqüência cardíaca, em batimentos por minuto (bpm), na potência de 40 Watts do teste de esforço físico dinâmico descontínuo do tipo degrau, para o grupo de voluntárias jovens $(n=10)$, pósmenopausa sem reposição hormonal (PMSRH) $(n=11)$ e pós-menopausa com reposição hormonal (PMCRH) $(n=8)$. Nível de significância de $\alpha=0,05$.

FIGURA 23. Em A, valores do tempo de variação da freqüência cardíaca, em segundos (s) e em B, valores de variação da freqüência cardíaca, em batimentos por minuto (bpm), na potência de 45 Watts do teste de esforço físico dinâmico descontínuo do tipo degrau, para o grupo de voluntárias jovens $(n=10)$, pósmenopausa sem reposição hormonal $(P M S R H)(n=8)$ e pós-menopausa com reposição hormonal (PMCRH) ( $n=7)$. Nível de significância de $\alpha=0,05$.

FIGURA 24. Em A, valores de freqüência cardíaca, em batimentos por minuto (bpm) e em B, dos índices de RMSSD dos intervalos R-R, em milissegundos (ms), para o grupo de voluntárias jovens $(n=10)$, na condição de repouso na posição sentada e durante as potências de 15 a 50 Watts (W) do teste de esforço físico dinâmico descontínuo do tipo degrau. Nível de significância de $\alpha=0,05$.

FIGURA 25. Em A, valores de freqüência cardíaca, em batimentos por minuto (bpm) e em B, dos índices de RMSSD dos intervalos R-R, em milissegundos (ms), para o grupo pós-menopausa sem reposição hormonal (PMSRH) $(n=13)$, na condição de repouso na posição sentada e durante as potências de 15 a 35 Watts (W) do teste de esforço físico dinâmico descontínuo do tipo degrau Nível de significância de $\alpha=0,05$.

FIGURA 26. Em A, valores de freqüência cardíaca, em batimentos por minuto (bpm) e em B, dos índices de RMSSD dos intervalos R-R, em milissegundos (ms), para o grupo de voluntárias pós-menopausa com reposição hormonal $(\mathrm{PMCRH})(\mathrm{n}=9)$, na condição de repouso na posição sentada e durante as potências de 15 a 35 Watts (W) do teste de esforço físico dinâmico descontínuo do tipo degrau. Nível de significância de $\alpha=0,05$ 
FIGURA 27. Valores dos índices de RMSSD dos intervalos R-R, em milissegundos (ms), para o grupo de voluntárias jovens $(n=10)$, pós-menopausa sem reposição hormonal (PMSRH) $(n=13)$ e pós-menopausa com reposição hormonal (PMCRH) $(n=9)$, durante a potência de 15 Watts do teste de esforço físico dinâmico descontínuo do tipo degrau. Nível de significância de $\alpha=0,05$.

FIGURA 28. Valores dos índices de RMSSD dos intervalos R-R, em milissegundos (ms), para o grupo de voluntárias jovens $(n=10)$, pós-menopausa sem reposição hormonal (PMSRH) $(n=13)$ e pós-menopausa com reposição hormonal (PMCRH) $(n=9)$, durante a potência de 20 Watts do teste de esforço físico dinâmico descontínuo do tipo degrau. Nível de significância de $\alpha=0,05$.

FIGURA 29. Valores dos índices de RMSSD dos intervalos R-R, em milissegundos (ms), para o grupo de voluntárias jovens $(n=10)$, pós-menopausa sem reposição hormonal (PMSRH) $(n=13)$ e pós-menopausa com reposição hormonal (PMCRH) $(n=9)$, durante a potência de 25 Watts do teste de esforço físico dinâmico descontínuo do tipo degrau. Nível de significância de $\alpha=0,05$.

FIGURA 30. Valores dos índices de RMSSD dos intervalos R-R, em milissegundos (ms), para o grupo de voluntárias jovens $(n=10)$, pós-menopausa sem reposição hormonal (PMSRH) $(n=13)$ e pós-menopausa com reposição hormonal (PMCRH) $(n=9)$, durante a potência de 30 Watts do teste de esforço físico dinâmico descontínuo do tipo degrau. Nível de significância de $\alpha=0,05$.

FIGURA 31. Valores dos índices de RMSSD dos intervalos R-R, em milissegundos (ms), para o grupo de voluntárias jovens $(n=10)$, pós-menopausa sem reposição hormonal (PMSRH) $(n=13)$ e pós-menopausa com reposição hormonal (PMCRH) $(n=9)$, durante a potência de 35 Watts do teste de esforço físico dinâmico descontínuo do tipo degrau. Nível de significância de $\alpha=0,05$. 
FIGURA 32. Valores dos índices de RMSSD dos intervalos R-R, em milissegundos (ms), para o grupo de voluntárias jovens $(n=10)$, pós-menopausa sem reposição hormonal (PMSRH) $(n=11)$ e pós-menopausa com reposição hormonal (PMCRH) $(n=8)$, durante a potência de 40 Watts do teste de esforço físico dinâmico descontínuo do tipo degrau. Nível de significância de $\alpha=0,05$.

FIGURA 33. Valores dos índices de RMSSD dos intervalos R-R, em milissegundos (ms), para o grupo de voluntárias jovens $(n=10)$, pós-menopausa sem reposição hormonal (PMSRH) $(n=8)$ e pós-menopausa com reposição hormonal (PMCRH) $(n=7)$, durante a potência de 45 Watts do teste de esforço físico dinâmico descontínuo do tipo degrau. Nível de significância de $\alpha=0,05$.

FIGURA 34. Em A, valores de potência em Watts do teste de esforço físico dinâmico descontínuo do tipo degrau e em B, dos índices de RMSSD dos intervalos R-R, em milissegundos (ms), no nível de esforço em que foi caracterizado o limiar de anaerobiose pelo modelo matemático e estatístico semiparamétrico (SPM), para o grupo de voluntárias jovens $(n=10)$, pósmenopausa sem reposição hormonal (PMSRH) $(n=13)$ e pós-menopausa com reposição hormonal (PMCRH) $(n=9)$. Nível de significância de $\alpha=0,05$

FIGURA 35. Valores do índice RMSSD dos intervalos R-R (IRR), em milissegundos ( $\mathrm{ms}$ ), nas potências do teste de esforço físico dinâmico descontínuo do tipo degrau em que foi determinado o limiar de anaerobiose pelo modelo matemático e estatístico semiparamétrico (SPM) e nas potências em que ocorreu significância estatística dos índices de RMSSD, para o grupo de voluntárias jovens $(n=10)$. Nível de significância de $\alpha=0,05$ 
FIGURA 36. Valores do índice RMSSD dos intervalos R-R (IRR), em milissegundos ( $m s)$, nas potências do teste de esforço físico dinâmico descontínuo do tipo degrau em que foi determinado o limiar de anaerobiose pelo modelo matemático e estatístico semiparamétrico (SPM) e nas potências em que ocorreu significância estatística dos índices de RMSSD, para o grupo de voluntárias pós-menopausa sem reposição hormonal $(P M S R H)(n=13)$. Nível de significância de $\alpha=0,05$

FIGURA 37. Valores do índice RMSSD dos intervalos R-R (IRR), em milissegundos ( $\mathrm{ms}$ ), nas potências do teste de esforço físico dinâmico descontínuo do tipo degrau em que foi determinado o limiar de anaerobiose pelo modelo matemático e estatístico semiparamétrico (SPM) e nas potências em que ocorreu significância estatística dos índices de RMSSD, para o grupo de voluntárias pós-menopausa com reposição hormonal $(\mathrm{PMCRH})(\mathrm{n}=9)$. Nível de significância de $\alpha=0,05$

FIGURA 38. Associação dos índices de RMSSD dos intervalos R$R$, em milissegundos ( $m s$ ), com as potências do teste de esforço físico dinâmico descontínuo do tipo degrau realizado pelas voluntárias jovens $(n=10)$. Nível de significância de $\alpha=0,05$.

FIGURA 39. Associação dos índices de RMSSD dos intervalos R$R$, em milissegundos ( $m s$ ), com as potências do teste de esforço físico dinâmico descontínuo do tipo degrau realizado pelas voluntárias pós-menopausa sem reposição hormonal (PMSRH), sendo que nas potências de 15 a 35 Watts: $n=13$, de 40 Watts: $n=11$ e em 45 Watts: $n=8$. Nível de significância de $\alpha=$ 0,05 .

FIGURA 40. Associação dos índices de RMSSD dos intervalos R$R$, em milissegundos ( $m s$ ), com as potências do teste de esforço físico dinâmico descontínuo do tipo degrau realizado pelas voluntárias pós-menopausa com reposição hormonal (PMCRH), sendo que nas potências de 15 a 35 Watts: $n=9$, de 40 Watts: $n=8$ e em 45 Watts: $n=7$. Nível de significância de $\alpha=0,05$ 
FIGURA 41. Associação dos índices de RMSSD dos intervalos R$\mathrm{R}$, em milissegundos ( $\mathrm{ms}$ ), com as potências do teste de esforço físico dinâmico descontínuo do tipo degrau realizado pelas voluntárias jovens $(n=10)$, pósmenopausa sem reposição hormonal (PMSRH) $(n=13)$ e pós-menopausa com reposição hormonal (PMCRH) $(n=9)$, sendo que na potência de 40 Watts, PMSRH: $n=11$ e PMCRH: $n=8$; e em 45 Watts: PMSRH: $n=8$ e PMCRH: $n=7$. Nível de significância de $\alpha=0,05$.

FIGURA 42. Associação entre os valores dos índices de RMSSD dos intervalos R-R, em milissegundos (ms), nos níveis de potência do teste de esforço físico dinâmico descontínuo do tipo degrau, em que ocorreu o limiar de anaerobiose, pelo modelo matemático e estatístico semiparamétrico (SPM) no eixo X e em Y, no nível de potência em que ocorreu significância estatística dos índices RMSSD, para as voluntárias jovens $(n=10)$, pós-menopausa sem reposição hormonal (PMSRH) $(n=13)$ e pós-menopausa com reposição hormonal (PMCRH) ( $n=9)$, sendo que na potência de 40 Watts, PMSRH: $n=11$ e PMCRH: $n=8$; e em 45 Watts: PMSRH: $n=8$ e PMCRH: $n=7$. Nível de significância de $\alpha=0,05$. 


\section{LISTA DE TABELAS}

TABELA 1. Idade, características antropométricas e dados de freqüência cardíaca (FC), em bpm, de pressão arterial sistólica (PAS) e diastólica (PAD), em $\mathrm{mmHg}$, do tempo de ciclo menstrual, em dias, e tempo de pósmenopausa e de terapia de reposição hormonal (TRH), em anos, das voluntárias estudadas jovens, pósmenopausa sem reposição hormonal (PMSRH) e pósmenopausa com reposição hormonal (PMCRH)

TABELA 2. Dados dos valores de referência dos níveis hormonais e dos valores observados nas voluntárias pósmenopausa com reposição hormonal (PMCRH) e pósmenopausa sem reposição hormonal (PMSRH)

TABELA 3. Resultado dos exames laboratoriais de hemograma; urina tipo 1; glicemia, uréia e creatinina, em $\mathrm{mg} / \mathrm{dl}$; triglicérides, colesterol total, LDL, HDL, em $\mathrm{mg} / 100 \mathrm{ml}$ e $\mathrm{TSH}$, em $\mathrm{mUl} / \mathrm{ml}$ das voluntárias jovens, pósmenopausa sem reposição hormonal (PMSRH) e pósmenopausa com reposição hormonal (PMCRH).

TABELA 4. Velocidade, em mph, e porcentagem (\%) de inclinação da esteira, valores de freqüência cardíaca ( $F C)$, em bpm, pressão artéria sistólica (PAS) e diastólica (PAD), em $\mathrm{mmHg}$, atingidos no pico do teste de exercício físico dinâmico contínuo de avaliação cardiológica e valores de freqüência cardíaca máxima prevista para a idade (FC máx prev), em bpm, das voluntárias jovens, pós-menopausa sem reposição hormonal (PMSRH) e pós-menopausa com reposição hormonal $(\mathrm{PMCRH})$.

TABELA 5. Tipo de medicação hormonal utilizada pelas voluntárias pós-menopausa com reposição hormonal $(n=9)$ 


\section{LISTA DE ABREVIATURAS E SIGLAS}

\begin{tabular}{|c|c|}
\hline bpm & Batimentos por minuto \\
\hline $\mathrm{CO}_{2}$ & Dióxido de carbono \\
\hline DP & Desvio padrão \\
\hline ECG & Eletrocardiograma \\
\hline FC & Frequencia cardíaca \\
\hline FSH & Hormônio folículo estimulante \\
\hline HDL & Lipoproteína de alta densidade \\
\hline IMC & Índice de massa corporal \\
\hline Kg & Kilogramas \\
\hline $\mathrm{Kg} / \mathrm{m}^{2}$ & Kilogramas por metro quadrado \\
\hline LA & Limiar de anaerobiose \\
\hline LDL & Lipoproteína de baixa densidade \\
\hline m & Metros \\
\hline MC5 & $\begin{array}{l}\text { Sistema de derivação para eletrocardiograma (eletrodo } \\
\text { negativo no manúbrio esternal, eletrodo positivo no } 5^{\circ} \\
\text { espaço intercostal na linha axilar anterior à direita e o neutro } \\
\text { no } 5^{\circ} \text { espaço intercostal na linha axilar anterior à esquerda) }\end{array}$ \\
\hline mg & Miligramas \\
\hline $\mathrm{mg} / 100 \mathrm{ml}$ & Miligramas por 100 mililitros \\
\hline $\mathrm{mg} / \mathrm{dl}$ & Miligramas por decilitro \\
\hline $\mathbf{m m H g}$ & Milímetro de mercúrio \\
\hline mph & Milhas por hora \\
\hline ms & Milissegundos \\
\hline $\mathrm{mUl} / \mathrm{ml}$ & Micro unidades internacionais por mililitro \\
\hline $\mathbf{N}$ & Número de intervalos $\mathrm{R}-\mathrm{R}$ na série de dados selecionados \\
\hline PA & Pressão arterial \\
\hline PAD & Pressão arterial diastólica \\
\hline PAS & Pressão arterial sistólica \\
\hline $\mathrm{pg} / \mathrm{ml}$ & Picogramas por mililitro \\
\hline
\end{tabular}




\begin{tabular}{|c|c|}
\hline PMCRH & Grupo pós-menopausa com reposição hormonal \\
\hline PMSRH & Grupo pós-menopausa sem reposição hormonal \\
\hline RMSSD & $\begin{array}{l}\text { Raiz quadrada da média dos quadrados das diferenças entre } \\
\text { os intervalos } R-R \text { normais sucessivos }\end{array}$ \\
\hline RR & Intervalos R-R \\
\hline $\mathbf{s}$ & Segundos \\
\hline SPM & Modelo matemático e estatístico semiparamétrico \\
\hline TEFDD-D & Teste de esforço físico dinâmico descontínuo do tipo degrau \\
\hline TSH & Hormônio estimulante da tireóide \\
\hline VFC & Variabilidade da freqüência cardíaca \\
\hline w & Watts \\
\hline $\mathbf{X}$ & Média \\
\hline
\end{tabular}




\section{RESUMO}

Ferreira, V. A influência da idade e da reposição hormonal sobre a modulação autonômica do coração e o limiar de anaerobiose. São Carlos, 2003. 120p. Dissertação (Mestrado) - Escola de Engenharia de São Carlos / Faculdade de Medicina de Ribeirão Preto / Instituto de Química de São Carlos, Universidade de São Paulo.

Este trabalho teve por objetivo avaliar a modulação autonômica da freqüência cardíaca (FC) durante o repouso, nas posições supina e sentada, e durante teste de esforço físico dinâmico descontínuo do tipo degrau (TEFDD-D) em mulheres jovens e pós-menopausa sem (PMSRH) e com reposição hormonal (PMCRH); determinar o limiar de anaerobiose (LA) a partir da análise das respostas de FC e pela análise dos índices de RMSSD (raiz quadrada da média dos quadrados das diferenças entre os intervalos R-R normais sucessivos), em milissegundos (ms), e comparar o grau de correlação entre estas duas metodologias de análise. Foram estudadas 11 jovens (24 $\pm 2,77$ anos), 13 PMSRH $(57 \pm 5,28)$ e 9 PMCRH $(55 \pm 5,40$ anos). O TEFDD-D foi realizado em cicloergômetro, sendo iniciado com a potência de $15 \mathrm{~W}$ e com incrementos de 5 em $5 \mathrm{~W}$. A FC (bpm) e os intervalos R-R (ms) foram captados em tempo real, por um período de 360s em repouso, em cada posição, e durante 60 s em repouso sentado no cicloergômetro, 240s em exercício e 60s em recuperação, em cada potência do TEFDD-D. Foram calculados as médias da FC (bpm) e os índices de RMSSD dos intervalos $R-R$ (ms) para as condições de repouso e durante 180s do exercício nas potências estudadas; cálculo da variação da FC (bpm) no início do exercício e do tempo (s) desta variação. A determinação do LA foi feita pelo ajuste do modelo matemático e estatístico semiparamétrico (SPM) aos dados de FC e pelos índices de RMSSD dos intervalos R-R (ms). Os testes estatísticos utilizados foram: Wilcoxon, Kruskall-Wallis, Friedman, Dunn e o teste de correlação de Spearman, nível de significância de $5 \%$. Durante o repouso, as jovens apresentaram valores dos índices de RMSSD significativamente $(p<0,05)$ superiores em relação aos outros 2 grupos. As 
variações da FC das jovens no início do exercício foram maiores que as dos grupos PMSRH e PMCRH, enquanto que o tempo de variação da $\mathrm{FC}$ foi similar entre os 3 grupos. Na transição do repouso para o exercício, a FC aumentou progressivamente, enquanto que a variabilidade da freqüência cardíaca (VFC) diminuiu. Na comparação intergrupo dos índices de RMSSD, obtidos em cada nível de potência, foi observada diferença significativa $(p<0,05)$ apenas em $35 \mathrm{~W}$. Tanto pelo modelo SPM, como pela análise dos índices de RMSSD, as jovens atingiram o LA em potências superiores comparativamente as PMSRH e PMCRH. Os grupos PMSRH e PMCRH apresentaram resultados similares. Não foram observadas diferenças significativas $(p>0,05)$ na comparação dos 2 métodos. $O$ teste de correlação de Spearman mostrou uma associação significativa $(p<0,05)$ entre os mesmos. Estes dados sugerem que após a menopausa ocorre uma diminuição da modulação vagal sobre o coração tanto em repouso como durante o exercício físico, decorrente do processo do envelhecimento e da redução da capacidade física. A terapia de reposição hormonal não teve nenhuma influência sobre os resultados. As duas metodologias de análise do LA se mostraram similares, sugerindo que a mudança de inclinação da resposta da FC ocorre em níveis de esforço em que a VFC se encontra significativamente reduzida.

Palavras-chave: freqüência cardíaca; variabilidade da freqüência cardíaca; sistema nervoso autônomo; menopausa; terapia de reposição hormonal. 


\section{ABSTRACT}

Ferreira, V. Influence of age and hormonal replacement on the autonomic modulation of the heart and the anaerobiosis threshold. São Carlos, 2003. 120 p. Dissertação (Mestrado) - Escola de Engenharia de São Carlos / Faculdade de Medicina de Ribeirão Preto / Instituto de Química de São Carlos, Universidade de São Paulo.

The objectives of the present study were to assess the autonomic modulation of the heart rate (HR) at rest, in the supine and sitting position, and during a step type discontinuous dynamic physical effort (STDDPE) in young and postmenopausal women not receiving (PMWtHR) and receiving hormonal replacement treatment $(\mathrm{PMWHR})$; to determine the anaerobiosis threshold (AT) based on the analysis of $H R$ response and the RMSSD indices (square root of the mean squared differences of successive $R-R$ intervals), in milliseconds (ms), and to compare the degree of correlation between these two analysis methodologies. The study was conducted on 11 young women (24 \pm 2.77 years), 13 PMWtHR (57 \pm 5.28$)$ and 9 PMWHR (55 \pm 5.40 years). The STDDPE was performed on a bicycle ergometer at an initial power of $15 \mathrm{~W}$, followed by power increments of $5 \mathrm{~W}$. HR (bpm) and $\mathrm{R}-\mathrm{R}$ intervals (ms) were obtained in real time over a period of $360 \mathrm{~s}$ under resting conditions in each position, during $60 \mathrm{~s}$ in the sitting rest position on the bicycle ergometer, $240 \mathrm{~s}$ during exercise and $60 \mathrm{~s}$ during recuperation at each STDDPE power. Mean HR (bpm) and RMSSD indices of the R-R intervals (ms) were calculated for the resting condition and during $180 \mathrm{~s}$ of exercise in the powers studied; the HR variation (bpm) and its time (s) were also calculated in the beginning of exercise. AT was determined by the semiparametric mathematical and statistical model (PMS) and by the RMSSD indices of the R-R intervals (ms). Data were analyzed statistically by the Wilcoxon, Kruskal-Wallis, Friedman, Dunn and Spearman correlation tests, with the level of significance set at $5 \%$. During rest, young women presented significantly higher RMSSD indices $(p<0.05)$ than the other 2 groups. The HR variations in young women in the beginning of the exercise 
were higher than the ones from the PMWtHR and PMWHR groups, whereas HR variations time was similar for the 3 groups. During the transition from rest to exercise HR increased progressively and HRV decreased. Intergroup comparison of RMSSD indices, obtained in each level of power, showed a significant difference $(p<0.05)$ only at $35 \mathrm{~W}$ power. On both PMS model and RMSSD indices analysis, young women reached AT at a higher power compared to PMWtHR and PMWHR groups. The PMWtHR and PMWHR groups presented similar results. No significant differences $(p>0.05)$ were observed when the methods were compared. The Spearman correlation test showed a significant association $(p<0.05)$ between methods. These data suggest that after menopause there is a decrease in vagal modulation of the heart both at rest and during physical exercise due to the aging process and the reduction in physical capacity. Hormonal replacement therapy had no effect on the results. Both methodologies of AT analysis were similar, suggesting that the change in the HR response occurs in levels of effort in which the HRV is significantly reduced.

Key-words: heart rate; heart rate variability; autonomic nervous system; menopause; hormonal replacement therapy. 


\section{INTRODUÇÃO}

O sistema cardiovascular, formado pelo coração e pelos vasos sangüíneos, desempenha importantes funções fisiológicas em todos os organismos vivos. Sua principal função é o transporte de substâncias presentes no sangue, participando também da manutenção da temperatura corporal e do controle neuro-hormonal do organismo (GALLO Jr et al., 1996).

Os ajustes rápidos do sistema cardiovascular são modulados principalmente pelo sistema nervoso autônomo por meio da estimulação ou inibição das fibras nervosas simpática e parassimpática (nervo vago) e pelas substâncias químicas que circulam no sangue (ROWELL, 1986; MITCHELL, 1990).

As fibras nervosas eferentes simpáticas possuem terminações nervosas nos nódulos sinusal e atrioventricular, bem como nos músculos dos átrios e ventrículos. Sua estimulação libera as catecolaminas adrenalina e noradrenalina. Esses hormônios neurais aumentam a freqüência dos batimentos cardíacos e a força de contração do miocárdio. As catecolaminas circulantes liberadas pelas glândulas supra-renais em resposta a ativação simpática generalizada, também produzem um efeito semelhante, porém de ação mais lenta, sobre a função cardíaca. Já as terminações nervosas das fibras parassimpáticas (nervo vagos) se concentram nos nódulos sinusal e 
atrioventricular e nos músculos atriais. Sua estimulação libera acetilcolina, que retarda o ritmo de descarga sinusal, diminuindo a freqüência cardíaca (FC) (ROWELL, 1986; McARDLE et al., 1998).

Os vasos sangüíneos, mais especificamente as pequenas artérias, arteríolas e esfíncteres pré-capilares, recebem inervação das fibras nervosas simpáticas adrenérgicas (noradrenalina) e colinérgicas (acetilcolina), que quando estimuladas, promovem vasoconstrição e vasodilatação, respectivamente. As veias recebem inervação das fibras nervosas simpáticas adrenérgicas, que ao serem estimuladas causam a venoconstrição. Devemos levar em consideração o fato de que qualquer vasoconstrição de ativação simpática que esteja presente em um tecido ativo é sobrepujada rapidamente pela poderosa vasodilatação induzida pelos co-produtos do metabolismo local (McARDLE et al., 1998).

Dessa forma, os ajustes do sistema cardiovascular permitem a regulação rápida da $\mathrm{FC}$ assim como a distribuição efetiva do sangue no circuito vascular em resposta às necessidades metabólicas e fisiológicas do organismo (McArdle et al., 1998).

A regulação da freqüência dos batimentos cardíacos pode ser em decorrência do controle intrínseco, fatores humorais e do sistema nervoso autônomo. Assim, a influência da estimulação ou inibição das fibras nervosas simpáticas e parassimpáticas, nas respostas da FC, sobrepõe ao ritmo inerente do miocárdio (TASKE FORCE, 1996). A complexa interação entre estas duas eferências resulta em oscilações da FC instantânea e dos 
intervalos $R-R$, as quais denominamos de variabilidade da freqüência cardíaca (VFC) (LONGO et al., 1995; TASKE FORCE, 1996).

Os principais mecanismos de controle cardiovascular responsáveis pelas flutuações da FC, ou seja, por sua variabilidade são: a respiração, a termo-regulação, o sistema renina-angiotensina, e a atividade barorreflexa. Estes mecanismos levam a contínuos ajustes da FC, adaptando-a às necessidades de cada momento (LONGO et al., 1995).

A VFC é um importante parâmetro de avaliação não-invasivo da integridade da função neurocardíaca (TASKE FORCE, 1996). Estudos têm mostrado que a diminuição na VFC é um poderoso preditor de mortalidade e complicações arrítmicas em pacientes pós-infarto agudo do miocárdio (KLEIGER et al., 1987; CRIPPS et al., 1991; BIGGER et al., 1992). Nos indivíduos com diabetes e hipertensão arterial também tem sido observada uma redução na VFC (MALPAS \& MALING, 1990; FAZAN Jr et al., 1997; SINGH et al., 1998; BARBOSA FILHO et al., 2002). Há indícios de que esta redução esteja relacionada a um aumento na atividade simpática ou a uma diminuição da atividade vagal (TASKE FORCE, 1996).

A VFC pode ser estudada de múltiplas maneiras. Os métodos mais comumente utilizados são a análise no domínio do tempo e a análise no domínio da freqüência (análise espectral). Qualquer que seja o método, esta pode ser baseada em registros de curta duração (5 a 30 minutos) ou de longa duração (24 horas) (LONGO et al., 1995; TASKE FORCE, 1996).

$\mathrm{Na}$ análise no domínio do tempo, a FC instantânea e os intervalos R$\mathrm{R}$ são determinados e posteriormente analisados por meio de cálculos 
estatísticos. Neste estudo foi utilizado o índice de RMSSD dos intervalos RR (raiz quadrada da média dos quadrados das diferenças entre os intervalos $\mathrm{R}-\mathrm{R}$ normais sucessivos) por ser um dos índices recomendados para este tipo de análise e também por suas propriedades estatísticas (TASKE FORCE, 1996).

A determinação dos valores normais de VFC representa importante área de estudos, servindo de base para avaliar alterações da mesma, nas diversas doenças que, direta ou indiretamente, afetam o coração (AMERICAN COLLEGE OF CARDIOLOGY CARDIOVASCULAR TECHNOLOGY ASSESSMENT COMMITTEE, 1993). Para isso, diversos estudos foram e estão sendo realizados em diferentes condições, tais como: repouso, manobras autonômicas e durante exercício físico (GROSSMAN et al., 1991; AHMED et al., 1994; MUKAI \& HAYANO, 1995; BARBOSA et al., 1996; YAMASAKI et al., 1996; ALONSO et al., 1998; MARÃES et al., 2000a; 2000b; MASSIN et al., 2000; RIBEIRO et al., 2000; 2001a; ROSÁRIO et al., 2000; SILVA et al., 2001; CATAl et al., 2002).

O exercício físico é um comportamento que intensifica sobremaneira o funcionamento do sistema cardiovascular. Dois mecanismos são responsáveis pelo controle desse sistema: o mecanismo neural central, denominado de "comando central", originado a partir de impulsos descendentes provenientes do córtex motor e o mecanismo neural reflexo, originado a partir de impulsos ascendentes originados nas terminações nervosas musculares das fibras do grupo III, sensíveis às alterações mecânicas dos músculos (mecanorreceptores) e das fibras do grupo IV, 
sensíveis às alterações metabólicas dos músculos (metabolorreceptores). Estes impulsos incidem sobre a área cardiovascular situada na formação reticular do bulbo e esta, por meio dos eferentes simpáticos e parassimpáticos do sistema nervoso autônomo, faz os ajustes cardiovasculares necessários durante o exercício (MITCHELL, 1990).

Os impulsos descendentes do córtex motor, os quais estão relacionados com a atividade neural responsável pelo recrutamento de unidades motoras, juntamente com os impulsos ascendentes dos mecanorreceptores, fornecem informações à área cardiovascular do bulbo concernentes ao tipo e a intensidade de contração muscular, bem como a massa muscular envolvida na atividade física. Coletivamente os impulsos do comando central e os impulsos reflexos mediados pelos mecanorreceptores desencadeiam as repostas cardiovasculares e determinam o nível inicial da atividade simpática e parassimpática sobre o coração e os vasos sangüíneos. Já os metabolorreceptores influenciam a área cardiovascular do bulbo com algum atraso, uma vez que sua atividade aferente é dependente das alterações metabólicas que ocorrem no músculo ao longo do exercício. Em exercícios dinâmicos de baixa intensidade, eles podem até não ser ativados (MITCHELL, 1990).

Um terceiro mecanismo também pode influenciar os ajustes cardiovasculares, é o chamado "comando cardiodinâmico", por meio do qual o retorno venoso e o fluxo de $\mathrm{CO}_{2}$ aos pulmões originariam informações aferentes à área cardiovascular do bulbo (BARROS NETO, 1996). 
A magnitude de resposta das variáveis cardiovasculares ao exercício está na dependência de um grande número de fatores, tais como: idade, sexo, características antropométricas (peso e altura), fatores genéticos e psicológicos, doenças (tipo e gravidade), hábitos alimentares, condições ambientais (temperatura, umidade e pressão barométrica), nível de condicionamento físico e condições relacionadas ao próprio exercício (tipo de exercício - dinâmico e/ou estático; intensidade, forma e duração; quantidade de massa muscular envolvida e posição de decúbito) (GALLO Jr et al., 1995).

O exercício físico, além das alterações metabólicas, provoca importantes alterações autonômicas que influenciam o funcionamento cardiovascular (SATO et al., 1980; MACIEL et al., 1986; GALLO Jr et al., 1988; 1989; 1995; MITCHELL, 1990).

Diversos estudos, com ou sem bloqueio farmacológico, demonstraram que a resposta da FC durante o exercício dinâmico é mediada por modificações na atividade dos eferentes simpático e parassimpático e que a relativa contribuição destas eferências estão na dependência da intensidade e duração do esforço (ROBINSON et al., 1966; NORDENFELT, 1971; MACIEL et al., 1986; GALLO Jr et al., 1988; 1995; SEALS \& VÍCTOR, 1991; NÓBREGA \& ARAÚJO, 1993).

Trabalhos onde foi estudada a regulação autonômica da FC em diferentes estágios do exercício, demonstraram que em baixas potências de esforço, inferiores às correspondentes ao limiar de anaerobiose (LA), a retirada vagal parece ser o único mecanismo responsável pela taquicardia 
induzida pelo exercício. A retirada vagal ocorre rapidamente no início do exercício (10 a 20 segundos), seguido por uma tendência de estabilização após o primeiro minuto do exercício, mediada pela retomada vagal. Em potências de esforço acima do LA, o aumento lento e linear da FC se deve a estimulação simpática e esta estimulação aumenta de intensidade proporcionalmente ao aumento do nível de esforço. Vale ressaltar que a taquicardia que ocorre nos primeiros segundos do exercício físico, mediada pela retirada vagal, independe do nível de esforço (MACIEL et al., 1986; GALLO Jr et al., 1988; 1989; 1995; YAMAMOTO et al., 1991; NÓBREGA \& ARAÚJO, 1993; TULPPO et al., 1996)

ALONSO et al. (1998) estudando o efeito do impacto metabólico sobre o comportamento da FC e de sua variabilidade (VFC), durante exercício físico progressivo do tipo degrau máximo, verificaram um aumento da FC concomitante ao aumento da intensidade de esforço e uma diminuição progressiva da VFC até a intensidade do exercício em que foi caracterizado o LA, a partir deste ponto mantendo-se inalterada, sugerindo que a diminuição da VFC ocorre em fases do exercício em que ocorre a transição do predomínio do metabolismo aeróbio para o anaeróbio, o que sugere uma menor participação vagal sobre o nódulo sinusal.

Estudos têm demonstrado que a retirada vagal que ocorre no início do exercício é decorrente dos impulsos aferentes da região do córtex motor e dos mecanorreceptores sobre a área cardiovascular no bulbo (MITCHELL, 1990; NÓBREGA \& ARAÚJO, 1993; WILLIAMSON et al., 1995) enquanto que a intensificação simpática sobre o coração tem sido explicada pela ação 
das catecolaminas circulantes (CHRISTENSEN \& GALBO, 1983; ROWELL, 1986) e pela ação metabolorreflexa aferente iniciada na musculatura esquelética ativa (MITCHELL, 1990; MIDDLEKAUFF et al., 1997). Todos esses mecanismos agindo em sintonia para atender a demanda metabólica na musculatura esquelética ativa.

Como pode ser observado, o LA, além de ser um delimitador de dois estados fisiológicos marcadamente distintos, altera a atividade do sistema nervoso autônomo, principalmente da eferência simpática sobre o coração e os vasos sangüíneos, modificando as respostas cardiovasculares (GALLO Jr et al., 1996).

A determinação do LA pode ser feita de maneira invasiva, utilizando por exemplo, a lactacidemia. Neste método, o LA é caracterizado no nível de esforço em que for observado um aumento exponencial da curva de concentração sangüínea de lactato (COYLE et al., 1983; WELTMAN et al., 1990; FOSTER et al., 1999).

O LA também pode ser determinado de forma indireta, por meio da ergoespirometria, onde o mesmo é identificado no momento em que se observa um aumento desproporcional da ventilação pulmonar e da produção de dióxido de carbono, relativamente, à elevação linear do consumo de oxigênio (WASSERMAN et al., 1999).

Tem sido proposto outras técnicas, não-invasivas e de baixo custo para se identificar o LA, uma delas refere-se ao comportamento da FC durante exercício dinâmico e a outra ao comportamento de sua variabilidade (VFC). 
Analisando a FC durante exercício físico dinâmico é possível identificar a potência na qual ocorre a perda de sua estabilidade, caracterizando de forma indireta o LA (RIBEIRO et al., 2001b; FERREIRA et al., 2002; OLIVEIRA et al., 2002; SAKABE et al., 2002).

Pela análise da VFC, o LA é determinado na potência do exercício físico dinâmico em que se observa uma diminuição significativa da mesma em relação aos valores de repouso. (ALONSO et al., 1998; MARÃES et al., 2000c)

O processo do envelhecimento promove modificações autonômicas do controle cardiovascular, caracterizadas principalmente por uma diminuição do tônus vagal, e conseqüentemente, diminuição na VFC na condição de repouso (SCHWARTZ et al., 1991; BARBOSA et al., 1996; CATAI, 1999; CATAl et al., 2000; RIBEIRO, 2001; RIBEIRO et al., 2001a). Além disso, o envelhecimento leva à um declínio fisiológico das funções orgânicas, decorrentes principalmente das alterações estruturais que ocorrem nos sistemas biológicos com o passar da idade. Tais alterações levam a uma redução na capacidade física dos indivíduos, caracterizada principalmente pela redução de parâmetros cardiovasculares, tais como: consumo máximo de oxigênio, FC máxima e diferença arterio-venosa de oxigênio, quando o indivíduo é submetido à um exercício físico aeróbio (FLEG, 1986; WALSH, 1987; CATAI, 1999; CATAl et al., 2000).

$\mathrm{Na}$ mulher, o processo fisiológico do envelhecimento adquire características próprias em decorrência das alterações hormonais femininas, bem caracterizadas na fase após a menopausa. 
A menopausa ocorre, geralmente, entre 45 e 55 anos de vida, porém varia com a raça, com fatores hereditários, fatores sócio-econômicos, nutrição, desordens orgânicas e outros aspectos. Ela corresponde à última menstruação governada pelos ovários, entretanto, é necessário aguardar um ano de amenorréia para se firmar o diagnóstico de menopausa, em virtude das irregularidades menstruais comuns nesse período (HALBE, 1998; KENEMANS et al., 2001).

O hipoestrogenismo desse período determina uma série de alterações físicas, psicológicas e metabólicas, manifestando-se por sintomas vasomotores, distúrbios menstruais, sintomas decorrentes da atrofia urogenital e problemas psicológicos, com piora significativa da qualidade de vida das mulheres (STEINGOLD et al., 1985; BATHIA et al., 1989; HALBE, 1998; KENEMANS et al., 2001). No entanto, são os efeitos da deficiência estrogênica a longo prazo que mais preocupam, pois eles levam a comprometimento como as doenças cardiovasculares.

A incidência de doenças cardiovasculares é baixa na mulher antes da menopausa, aumentando significativamente o seu aparecimento após este período. (GENSINI et al., 1996; MENDELSOHN, 2000). Segundo SULLIVAN \& FOWLKES (1996) o uso da terapia de reposição estrogênica pode levar a uma redução de até 50\% na incidência destas doenças.

Os mecanismos responsáveis pela ação profilática dos estrogênios não são completamente conhecidos. Até recentemente eles eram atribuídos a ação deste hormônio sobre a promoção de um perfil lipoprotéico antiaterogênico, caracterizado principalmente por uma diminuição da 
lipoproteína de baixa densidade (LDL) e aumento da lipoproteína de alta densidade (HDL). No entanto, outros mecanismos devem ser incluídos, como: a ação direta sobre os receptores e enzimas da parede arterial diminuindo a pressão e melhorando o fluxo sangüíneo, a estimulação da síntese de prostaciclina e óxido nítrico que atuam como vasodilatadores, a inibição do depósito de colesterol e LDL-oxidado na parede dos vasos sangüíneos, a redução na proliferação da musculatura lisa arterial induzida pelas lipoproteínas, a inibição da proliferação da íntima associada a lesões mecânicas do endotélio e a estimulação dos anti-agregantes plaquetários (GENSINI et al., 1996; PINES et al., 1997; ESPELAND, 1998; HALBE, 1998; MENDELSOHN, 2000; SANADA et al., 2000).

Os estrogênios também parecem influenciar a modulação autonômica do coração. MERCURO et al. (2000) observaram modificações na atividade vago-simpática de um grupo de mulheres após a menopausa, com redução dos índices de VFC no domínio do tempo e da freqüência.

DAVY et al. (1998) também observaram diminuição da VFC nas mulheres na fase após a menopausa, tanto nas sedentárias como nas mulheres que praticavam atividade física, sendo que nestas últimas a redução foi menor.

Nos estudos de RIBEIRO (2001) e RIBEIRO et al. (2001a) também foi verificado menor valor dos índices temporais, RMSM e RMSSD de VFC, na condição de repouso e durante exercício físico dinâmico de mulheres na fase após a menopausa em comparação às mulheres jovens. Foi sugerido 
que a redução da VFC observada poderia estar relacionada à idade, à condição física das voluntárias e provavelmente aos fatores hormonais.

Em trabalho com animais (ratas fêmeas) McCABE et al. (1981) observaram uma diminuição da modulação vagal dessas após submetê-las a ooforectomia e sua normalização quando as ratas foram submetidas à reposição hormonal com estrógeno.

A terapia de reposição hormonal com estrógeno parece alterar favoravelmente o desbalanço autonômico que ocorre após a menopausa. HUIKURI et al. (1996), ROSANO et al. (1997), MERCURO et al. (2000) e VIRTANEN et al. (2000) verificaram um aumento na VFC de mulheres na fase após a menopausa sob tratamento de reposição hormonal com estrógeno. Entretanto, no estudo de FARAG et al. (2002), os resultados mostraram que o uso combinado de estrógeno e progesterona foi mais favorável à modulação autonômica do coração do que o estrógeno sozinho.

No entanto, outros estudos não observaram alterações nos índices de VFC em mulheres em uso de terapia de reposição hormonal com estrógeno (DAVY et al., 1998; CHRIST et al., 1999) ou de estrógeno com progesterona (NISKANEN et al., 2002). Além disso, CHRIST et al. (1999) observaram inclusive uma diminuição da VFC em um dos grupos de mulheres estudadas, submetidas à terapia combinada, ou seja, estrógeno associado a progesterona.

Após a menopausa também tem sido observado um declínio na capacidade física das mulheres, mas isto parece estar mais relacionado com o processo do envelhecimento do que com a deficiência estrogênica, como 
foi observado nos estudos de COWAN \& GREGORY (1985) e NOTELOVITZ et al. (1986). RIBEIRO et al. (2001b) verificaram uma menor capacidade aeróbia das mulheres na fase após a menopausa durante exercício físico dinâmico em relação às jovens.

No entanto, no estudo realizado em mulheres que apresentavam ciclos menstruais regulares foi observado menor concentração de lactato sangüíneo e conseqüentemente maior tolerância ao esforço durante o exercício realizado na fase lútea, quando comparado à fase pré-folicular, que apresenta baixos níveis estrogênicos (JURKOWSKI et al., 1981). Porém, outros autores não observaram diferenças no desempenho físico de mulheres jovens nas diferentes fases do ciclo menstrual (De SOUZA et al., 1990; BEMBEN et al., 1995).

Como pode ser observado, os estudos realizados no sentido de compreender as alterações na modulação autonômica do coração e na capacidade física em mulheres na fase após a menopausa, bem como o efeito da terapia de reposição hormonal sobre estas variáveis, permanecem sem elucidação definitiva. Dessa forma, torna-se imprescindível que trabalhos sejam realizados no sentido de trazer contribuições mais conclusivas neste assunto que é de extrema importância, uma vez que nesta fase da vida, as mulheres estão mais vulneráveis ao acometimento de doenças cardiovasculares. 


\section{OBJETIVO}

Esta pesquisa foi desenvolvida com os seguintes objetivos:

- Avaliar a modulação autonômica da freqüência cardíaca durante o repouso, nas posições supina e sentada, e durante o teste de esforço físico dinâmico descontínuo do tipo degrau, em mulheres jovens e na fase após a menopausa, com e sem reposição hormonal;

- Determinar o LA a partir da aplicação de modelo matemático e estatístico semiparamétrico (SPM) e pelos índices de RMSSD dos intervalos $\mathrm{R}-\mathrm{R}$;

- Comparar o grau de correlação entre o modelo SPM e os índices de RMSSD dos intervalos R-R. 


\section{MATERIAIS E MÉTODOS}

\subsection{Voluntárias}

No presente trabalho, foram estudadas 32 voluntárias do sexo feminino, clinicamente saudáveis, com padrão de vida sedentário e que apresentassem características antropométricas semelhantes para que houvesse homogeneidade da amostra, dentro de cada grupo.

As 32 voluntárias foram divididas em 3 grupos, a saber:

- Grupo Jovem: 10 mulheres na faixa etária de 20 a 30 anos (média = $24 \pm D P=2,77)$, com ciclo menstrual regular e que não faziam uso de anticoncepcionais, há pelo menos 6 meses, ou qualquer outro tipo de medicação.

- Grupo pós-menopausa sem reposição hormonal (PMSRH): 13 mulheres na faixa etária de 50 a 60 anos (média $=57 \pm \mathrm{DP}=5,28$ ), na fase após a menopausa, que não faziam uso de terapia de reposição hormonal e/ou qualquer outro medicamento que pudesse interferir nas respostas coletadas durante a pesquisa.

- Grupo pós-menopausa com reposição hormonal (PMCRH): 9 mulheres na faixa etária de 50 a 60 anos (média $=55 \pm \mathrm{DP}=5,40$ ), 
na fase após a menopausa, utilizando terapia de reposição hormonal, há pelo menos 6 meses e sem uso de outros medicamentos.

\subsection{Critérios de inclusão das voluntárias}

Para a inclusão das voluntárias nesta pesquisa, foram considerados os seguintes critérios:

- Sedentarismo, ou seja, que não praticassem atividades físicas sistemáticas e regulares nos últimos 12 meses;

- Não tabagistas;

- Não etilistas;

- Não usuárias de drogas que causem dependência química;

- Índice de massa corporal inferior a $30 \mathrm{Kg} / \mathrm{m}^{2}$;

- Ausência de anormalidades do sistema cardiovascular, respiratório ou músculo-esquelético;

- Ausência de alterações metabólicas, disfunções hormonais e ginecológicas;

- Sem história clínica de doença de Chagas, diabetes melito, dislipidemia e hipertensão arterial;

- Não usuárias de medicações anti-hipertensivas, cardioativas e antivertiginosas;

- Para as jovens: ciclos menstruais regulares nos últimos 12 meses antecedentes a participação no estudo;

- Para as voluntárias PMSRH e PMCRH: ausência de fluxo menstrual espontâneo há mais de 12 meses consecutivos, antes da participação 
no estudo; concentração sérica de $\mathrm{FSH}$ entre 23 e $116 \mathrm{mUl} / \mathrm{ml}$; concentração sérica de estradiol até $30 \mathrm{pg} / \mathrm{ml}$ para as PMSRH e até $93 \mathrm{pg} / \mathrm{ml}$ para as $\mathrm{PMCRH}$.

A seleção das voluntárias na fase após a menopausa foi realizada nos ambulatórios de Ginecologia Geral e Climatério do Centro de Saúde Escola "Dr. Joel Domingues Machado" da Faculdade de Medicina de Ribeirão Preto, situado à Rua Teresina, n 690, Bairro Sumarezinho, Ribeirão Preto-SP.

\subsection{Aspectos Éticos}

Respeitando as normas de conduta em pesquisa experimental com seres humanos, este trabalho foi submetido e aprovado pelo Comitê de Ética em Pesquisa da Universidade Federal de São Carlos, protocolo nº 019/2001 (Apêndice 1), e pelo Comitê de Ética em Pesquisa do Hospital das Clínicas da Faculdade de Medicina de Ribeirão Preto da Universidade de São Paulo, protocolo $n^{0} 7966 / 2001$ (Apêndice 1). As voluntárias foram informadas e esclarecidas a respeito dos objetivos e da metodologia experimental que seriam submetidas, explicitando o caráter não invasivo dos mesmos. No caso de aceitação plena, elas assinavam um termo de consentimento formal de participação no estudo (Apêndice 1). 


\subsection{Avaliação Clínica}

Anteriormente à realização do protocolo experimental, as voluntárias foram submetidas a uma série de avaliações, que tinham por objetivo assegurar suas condições de saúde e diagnosticar possíveis alterações que contra-indicassem a participação na pesquisa.

Primeiramente, foi realizada uma avaliação constando de anamnese, onde foram colhidos informações pessoais referentes aos hábitos de vida e alimentar, história atual e pregressa de doenças e os antecedentes familiares. Durante esta avaliação, também foi realizado um exame físico completo, com mensuração da FC, pressão arterial (PA), peso e altura.

Em seguida, as voluntárias eram encaminhadas para realizar uma série de exames laboratoriais, como: hemograma, urina de rotina, glicemia de jejum, uréia e creatinina séricas, lipidograma completo (colesterol total e frações, triglicérides) e hormônio estimulante da tireóide (TSH) (Apêndice 2).

Para que a fase após a menopausa fosse realmente caracterizada, foi realizada também a dosagem plasmática dos hormônios folículo estimulante (FSH) e estradiol.

Além desses exames, as voluntárias foram submetidas a uma avaliação clínica, realizada por um médico cardiologista, onde foram submetidas a uma avaliação em repouso e outra em exercício físico dinâmico contínuo, para avaliação da capacidade funcional e do sistema cardiovascular e para diagnosticar possíveis alterações isquêmicas, hipertensão reativa e arritmias importantes desenvolvidas durante o esforço (Apêndice 2). 
$\mathrm{Na}$ avaliação em repouso na posição supina, foi realizado um eletrocardiograma (ECG) convencional de 12 derivações, tanto antes como após o teste de exercício físico dinâmico contínuo.

A avaliação em exercício físico dinâmico contínuo foi realizada em esteira elétrica automática (Imbramed) com protocolo do tipo degrau contínuo (Protocolo de Bruce modificado II). Durante esse teste as voluntárias foram monitorizadas nas derivações MC5, DII e V2 modificadas. Inicialmente, era feito um registro eletrocardiográfico nas condições de repouso em pé e em apnéia inspiratória. Em seguida, iniciava-se o primeiro estágio do protocolo com inclinação da esteira de $0 \%$ e velocidade de 1,7 milhas por hora (mph). Nos dois estágios seguintes, aumentava-se a inclinação em 5\% e mantinha-se a velocidade em 1,7 mph. Nos estágios seguintes, aumentava-se a inclinação em $2 \%$ e a velocidade em 0,8 mph do $3^{\circ}$ ao $7^{\circ}$ estágio e em $0,5 \mathrm{mph}$ do $7^{\circ}$ ao $9^{\circ}$ estágio (figura 1 ). Cada estágio tinha duração de 3 minutos. O teste era interrompido quando as voluntárias atingiam a exaustão física e/ou com o surgimento de sinais e/ou sintomas limitantes. A escolha desta versão modificada do Protocolo de Bruce ao invés do original se deve ao fato de que nesta, o incremento de trabalho nos estágios inicias se dá de maneira mais suave, permitindo igualmente tanto o diagnóstico como a avaliação da capacidade funcional das voluntárias. 


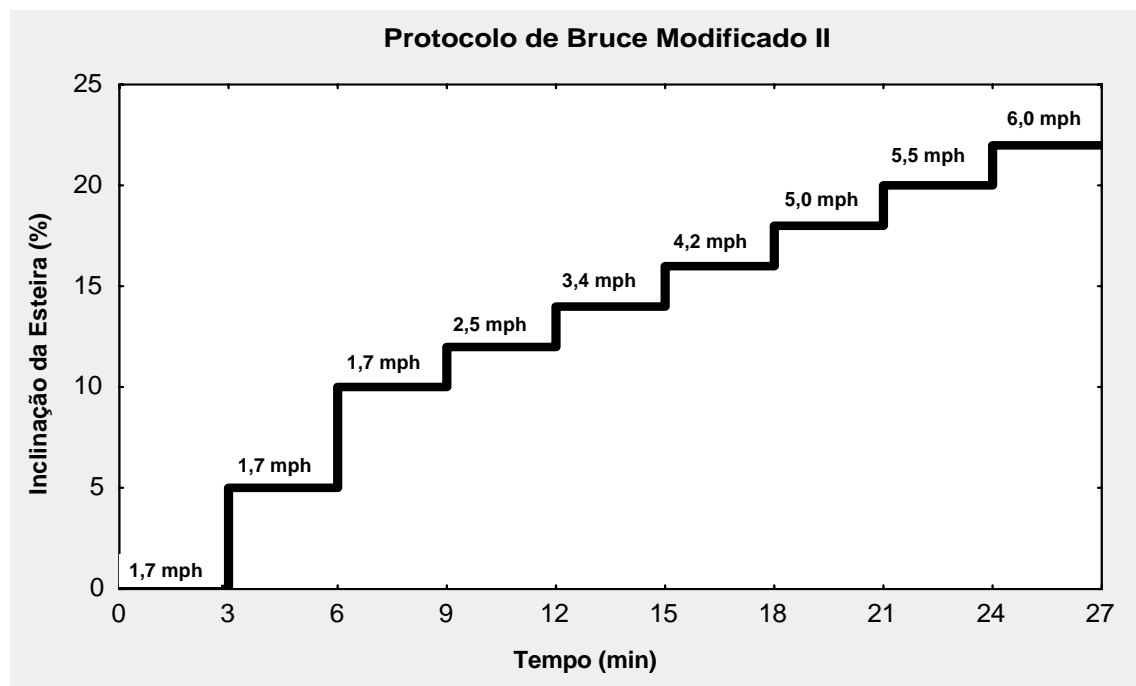

FIGURA 1. Representação esquemática do teste de exercício físico dinâmico contínuo, realizado em esteira elétrica automática com protocolo de Bruce modificado II.

\subsection{Procedimento Experimental}

Os experimentos foram realizados no Núcleo de Pesquisa em Exercício Físico (NUPEF) do Laboratório de Fisioterapia Cardiovascular da UFSCar e no Laboratório de Fisiologia do Exercício da Divisão de Cardiologia do Hospital das Clínicas da Faculdade de Medicina de Ribeirão Preto da USP, em um período específico, previamente marcado com as voluntárias, que foram informadas e esclarecidas antecipadamente sobre os procedimentos dos testes de esforço físico. As figuras 2 e 3 ilustram os equipamentos e a montagem experimental.

Com o propósito de não comprometer os resultados da pesquisa, bem como evitar intercorrências durante a realização dos testes, foram seguidos os seguintes procedimentos:

- Para a familiarização das voluntárias com o ambiente laboratorial (local, testes e equipamentos utilizados) foi realizada uma sessão 
prévia de visita ao laboratório, bem como a explicação dos procedimentos experimentais a serem realizados;

- Organização do laboratório e preparação dos materiais e equipamentos que seriam utilizados durante o experimento;

- Os testes foram realizados em sala climatizada por um condicionador de ar com controle da temperatura ambiente entre 21 e $23{ }^{\circ} \mathrm{C}$ e da umidade relativa do ar em torno de $60 \%$;

- Verificação das condições de saúde da voluntária no dia do teste: se ela teve uma boa noite de sono, se a mesma seguiu as orientações dadas no dia anterior, tais como: não ingerir bebidas alcoólicas e/ou estimulantes (café, chá, refrigerantes, etc.), não realizar qualquer tipo de esforço físico extenuante, fazer uma refeição leve pelo menos 2 horas antes do teste. Verificava-se, também, se as voluntárias estavam fazendo uso de roupas confortáveis e de sapatos apropriados para atividade física;

- Para o registro do ECG, foi utilizado eletrodo descartável e a pele era limpa com algodão e álcool para não haver interferências no registro;

- Antes de se iniciar o experimento, foi feito um repouso inicial de 15 minutos na posição supina e após isto se aferia a PA e a FC para verificar se as condições basais eram satisfatórias para dar início aos testes;

- A porta do laboratório era mantida fechada, com indicação de que o teste não deveria ser interrompido; 
- Procurava-se manter o mínimo trânsito de pessoas no laboratório (no máximo três pessoas);

- Procurava-se manter o máximo de silêncio durante os testes, falandose somente o necessário para a condução do experimento;

- Os testes foram realizados sempre num mesmo período do dia para evitar interferências de variações circadianas;

- Os testes de esforço físico dinâmico foram realizados em bicicleta ergométrica de frenagem eletromagnética (QUINTON - CORIVAL 400), com banco regulado de forma a permitir uma flexão de joelho em torno de 5 a 10 graus, e velocidade controlada por volta de 60 rotações por minuto;

- Durante os testes de esforço físico dinâmico, as voluntárias foram orientadas a não realizar contração isométrica ao segurar o guidão da bicicleta, não falar desnecessariamente e para que alertasse quanto ao aparecimento de qualquer sintoma que pudesse, eventualmente, vir a sentir durante a realização do mesmo. 


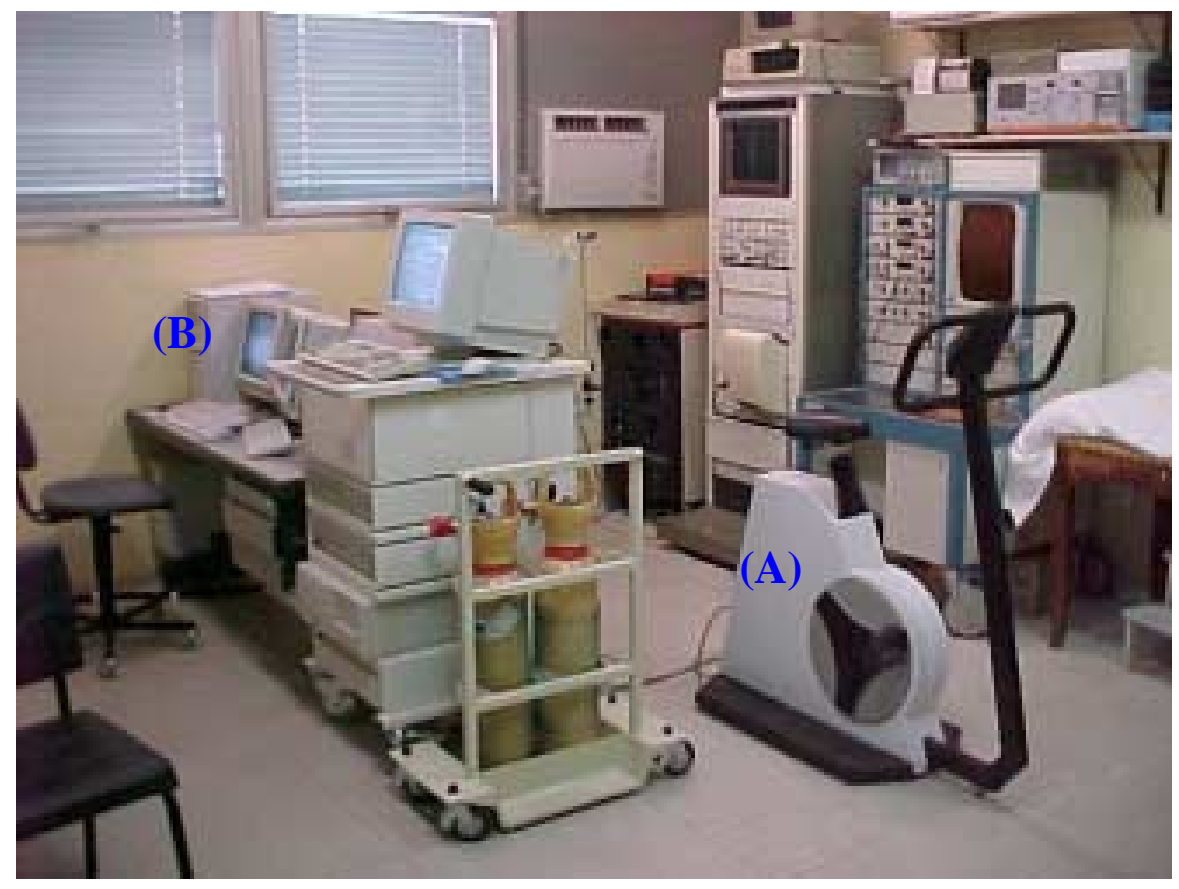

FIGURA 2. Ilustração dos equipamentos utilizados durante o teste de esforço físico dinâmico descontínuo do tipo degrau realizado no Laboratório de Fisiologia do Exercício da Divisão de Cardiologia do Hospital das Clínicas da Faculdade de Medicina de Ribeirão Preto da USP. Em A: bicicleta ergométrica de frenagem eletromagnética. Em $B$ : microcomputador com sistema de captação de sinal de eletrocardiograma. 


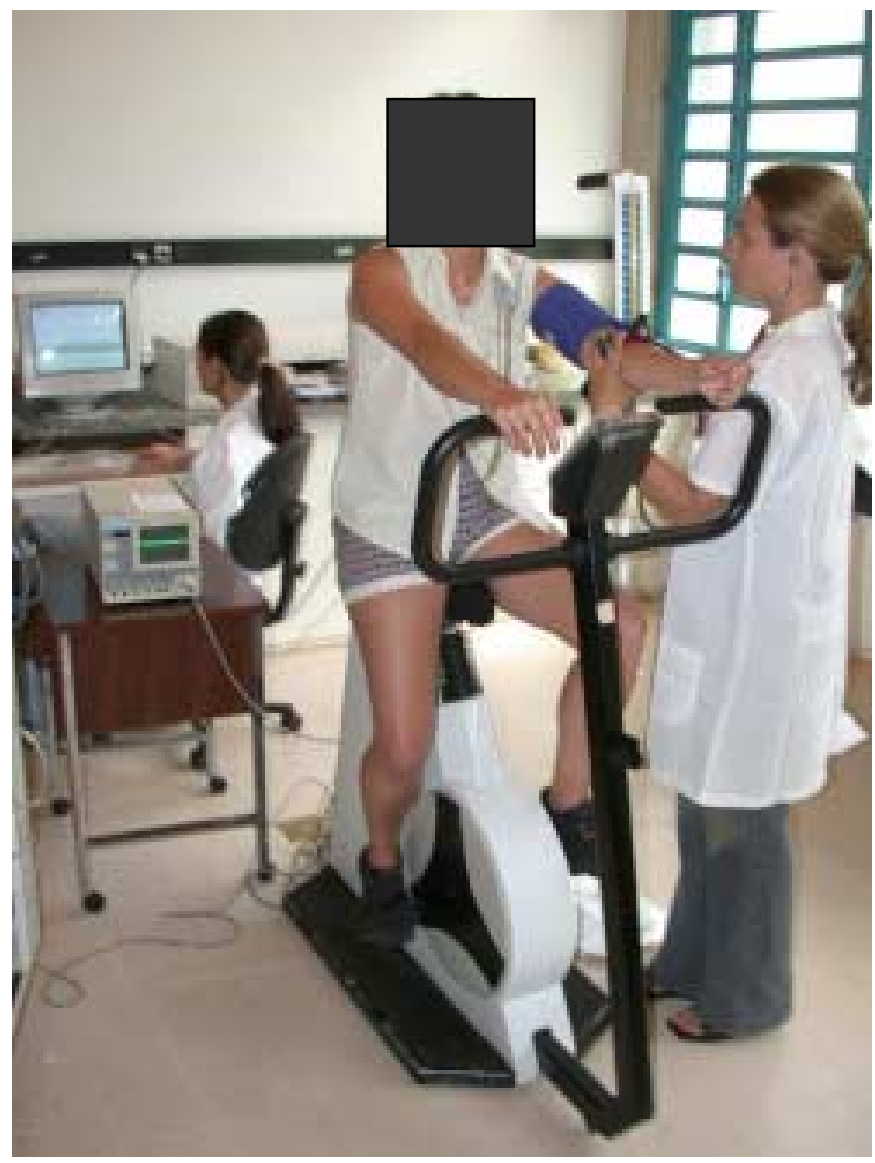

FIGURA 3. llustração do procedimento experimental durante o teste de esforço físico dinâmico descontínuo do tipo degrau realizado no Núcleo de Pesquisa em Exercício Físico (NUPEF) do Laboratório de Fisioterapia Cardiovascular da UFSCar. 


\subsection{Protocolo Experimental}

O protocolo experimental foi dividido em 2 etapas, realizadas no mesmo dia, durante as quais as voluntárias foram monitorizadas na derivação MC5 (pólo negativo no manúbrio esternal, pólo positivo no $5^{\circ}$ espaço intercostal na linha axilar anterior à esquerda e o neutro no $5^{\circ}$ espaço intercostal na linha axilar anterior à direita).

O ECG, os intervalos R-R e a FC foram captados e armazenados (figura 4) por meio de uma "interface" entre o monitor cardíaco (ECAFIX TC500) ou o Polígrafo (HP 7754B) e o microcomputador. O sinal analógico era digitalizado através de um conversor A/D e então processado por um "software" específico para captação do ECG e cálculos dos intervalos R-R (SILVA et al., 1994).

O grupo das voluntárias jovens realizou o protocolo experimental entre $\circ 6^{\circ}$ e $012^{\circ}$ dia do ciclo menstrual, que corresponde à fase folicular.

\subsubsection{Condição de Repouso}

Esta etapa foi realizada com o objetivo de avaliar a modulação autonômica do coração a partir da resposta da FC e de sua variabilidade durante a condição de repouso nas posições supina e sentada.

Para isso, a FC em batimentos por minuto (bpm), foi registrada batimento a batimento em tempo real durante 360 segundos (s) em cada posição. 


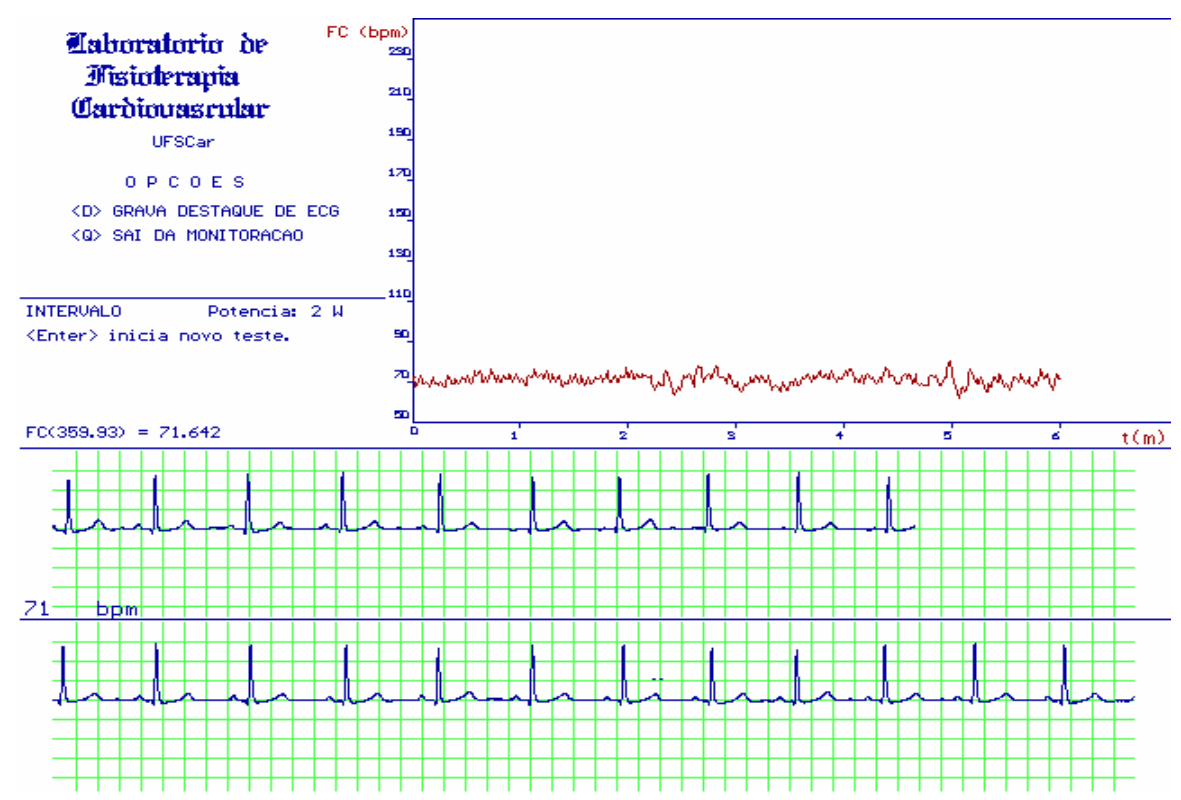

FIGURA 4. Ilustração da tela do computador do programa de aquisição do eletrocardiograma e da freqüência cardíaca, batimento a batimento, em tempo real, durante o repouso na posição sentada de uma das voluntárias jovens estudadas (ACM).

\subsubsection{Teste de esforço físico dinâmico descontínuo do tipo degrau (TEFDD-D)}

A aplicação do TEFDD-D teve por objetivo avaliar o comportamento da FC em resposta ao exercício físico dinâmico em potências submáximas de esforço, bem como a participação dos eferentes simpático e parassimpático na taquicardia induzida pelo exercício físico e ainda determinar o LA.

Este teste foi realizado em bicicleta ergométrica de frenagem eletromagnética, sendo iniciado com uma potência de 15 Watts (W) e com um incremento de potência progressivo de 5 em $5 \mathrm{~W}$ até que fosse visualizada a perda da estabilidade da resposta da FC. 
O protocolo constava de $60 \mathrm{~s}$ de repouso, $240 \mathrm{~s}$ em exercício e $60 \mathrm{~s}$ de recuperação. Entre as potências do TEFDD-D era interposto um período variável de recuperação até que a FC retornasse a valores próximos da condição inicial pré-esforço (figura 5).

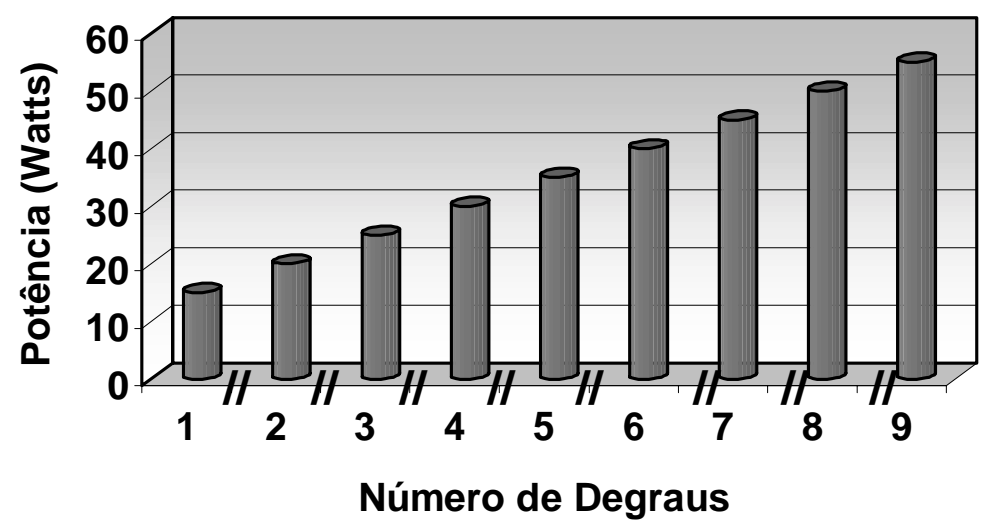

FIGURA 5. Representação esquemática do protocolo do teste de esforço físico dinâmico descontínuo do tipo degrau, com incrementos de potência progressiva de 5 em 5 Watts com duração de 240 segundos em cada nível de esforço, com períodos de repouso intercalados.

O TEFDD-D foi realizado em dia não consecutivo ao teste de exercício físico dinâmico contínuo de avaliação cardiológica. 


\subsection{Métodos de Análise}

3.7.1. Análise da resposta da freqüência cardíaca nas condições de repouso e durante 0 teste de esforço físico dinâmico descontínuo do tipo degrau

A FC captada e armazenada, batimento a batimento, em tempo real em repouso nas posições supina e sentada e durante cada potência do TEFDD-D foi calculada em média para as 33 voluntárias estudadas, utilizando-se para isso o aplicativo "STATISTICA for Windows, Release 5.5 StatSoft, Inc. (2000)".

No repouso, foi considerado para o cálculo o período de $360 \mathrm{~s}$ para cada uma das posições, supina e sentada. Já para o TEFDD-D foi considerado o período de 120 a 300 segundos de exercício, para cada nível de esforço.

\subsubsection{Variação da freqüência cardíaca e tempo de duração desta variação no início do exercício físico dinâmico descontínuo}

A partir dos dados de FC, obtidos batimento a batimento, em tempo real, e usando o sistema computacional integrado (SILVA et al., 1994) foi possível determinar o maior valor de FC ( FCpico), em bpm, e o instante ( $\left.\mathrm{t}_{1}\right)$, em s, em que este ocorreu no intervalo de 0 a $30 \mathrm{~s}$ no início do exercício, para cada uma das voluntárias nas potências de 15, 20, 25 30, 35, 40 e 45 W. 
Utilizando-se o aplicativo "STATISTICA for Windows, Release 5.5 StatSoft, Inc. (2000)" foi calculado a média da FC de repouso ( $\overline{F C} r e p)$, em bpm, captada nos $60 \mathrm{~s}$ iniciais que antecedem o exercício e a variação da freqüência cardíaca $(\triangle F C)$, em bpm, obtida a partir da diferença entre a FCpico atingida no intervalo de 0 a $30 \mathrm{~s}$ de exercício e a $\overline{F C}$ rep pré-esforço (Equação 1).

$$
\Delta F C=F C p i c o-\overline{F C} r e p \quad(\text { Equação } 1)
$$

Foi calculado também, o tempo de variação da $\mathrm{FC}\left(t_{/ \Delta F C}\right)$, em $\mathrm{s}$, que corresponde a diferença entre o instante em que ocorreu a FCpico $\left(\mathrm{t}_{1}\right) \mathrm{e}$ o tempo, em s, em que se iniciou o exercício ( $\mathrm{t}_{0}$ ) (Equação 2).

$$
t_{/ \triangle F C=t_{1}-t_{0} \quad \text { (Equação 2) }}
$$

\subsubsection{Variabilidade da freqüência cardíaca nas condições de repouso e durante o teste de esforço físico dinâmico descontínuo do tipo degrau}

A VFC em repouso, nas posições supina e sentada, e durante cada potência do TEFDD-D foi analisada no domínio do tempo, por meio do índice de RMSSD dos intervalos R-R, em milissegundos (ms), para as 33 voluntárias estudadas. 
O índice de RMSSD corresponde à raiz quadrada da somatória do quadrado da diferença dos intervalos $\mathrm{R}$ - $\mathrm{R}$, dividido pelo número de intervalos $R-R$ em $360 \mathrm{~s}$ de repouso (supino e sentado) e em $180 \mathrm{~s}$ (120 a $300 \mathrm{~s}$ ) durante o TEFDD-D, menos um (Equação 3) (ANTILA, 1979).

$$
R M S S D=\sqrt{\frac{\sum_{i=1}^{N-1}\left(R R_{i}-R R_{i+1}\right)^{2}}{N-1}} \quad \text { (Equação 3) }
$$

Onde:

$R R=$ intervalos $R-R$ e $\mathrm{N}$ = número de intervalos $\mathrm{R}-\mathrm{R}$ na série de dados selecionados.

Para obtenção deste índice, foi utilizado o programa "Analisador gráfico de intervalos R-R, freqüência cardíaca e eletrocardiograma", desenvolvido no Laboratório de Fisioterapia Cardiovascular da Universidade Federal de São Carlos (GOUVÊA et al., 1998), exemplificado na figura 6. 


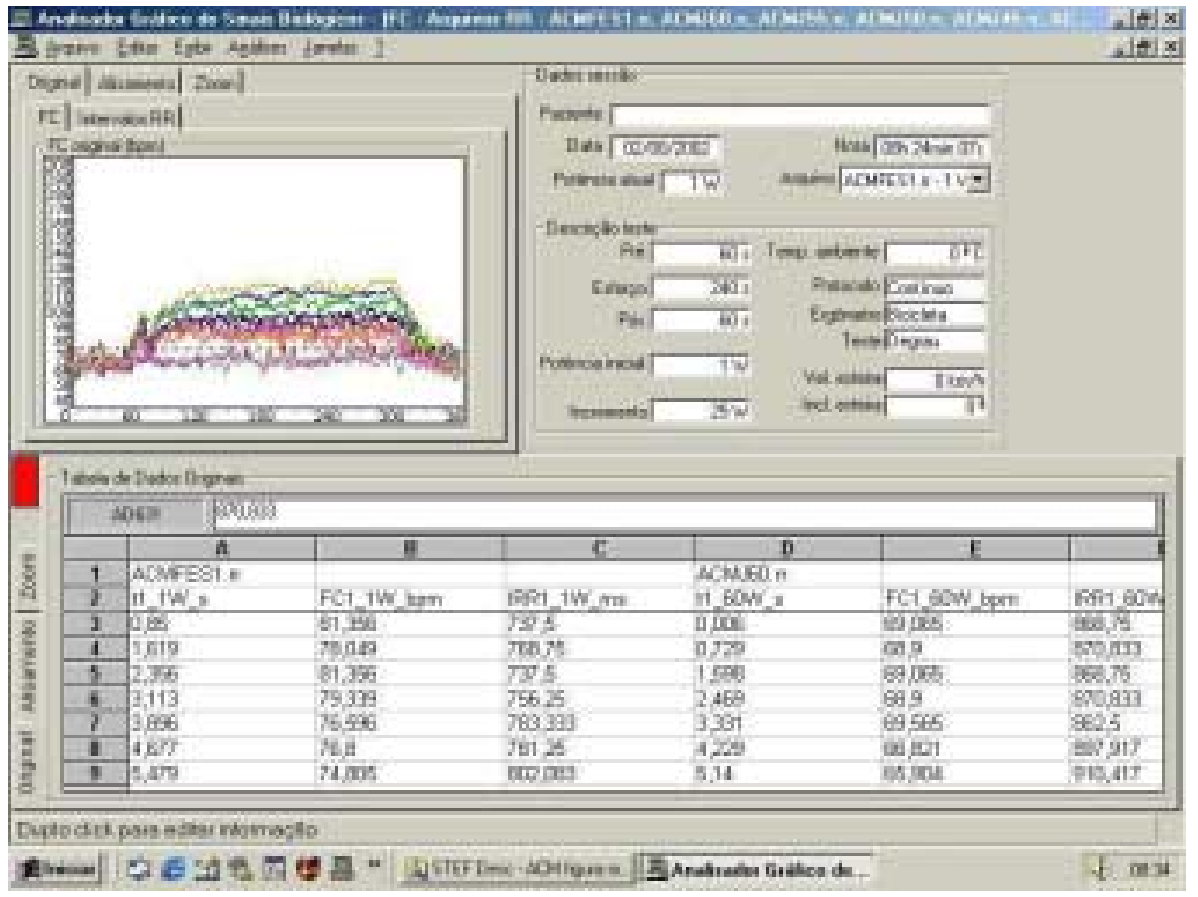

FIGURA 6. Ilustração da tela do computador do programa "Analisador gráfico de intervalos R-R, freqüência cardíaca e eletrocardiograma", contendo informações do protocolo utilizado, planilha de dados absolutos de freqüência cardíaca, batimento a batimento, intervalos $R-R$ e tempos correspondentes, gráficos das respostas da freqüência cardíaca em repouso e em exercício físico dinâmico. 


\subsubsection{Análise da resposta da freqüência cardíaca na determinação do limiar de anaerobiose por meio do modelo matemático e estatístico semiparamétrico}

O LA pelo método das respostas da FC pode ser caracterizado durante o TEFDD-D, como o momento em que uma reta ajustada aos seus valores admite uma inclinação positiva estatisticamente significante, o que tem sido atribuído ao início do predomínio da ativação simpática.

Para análise em questão, na seqüência da série de dados de FC, foi selecionado o período de 120 a 300 s de exercício, em cada potência do TEFDD-D, utilizando-se um modelo matemático e estatístico semiparamétrico (SPM), com uma rotina desenvolvida para este fim (OLIVEIRA et al., 2002) a partir do aplicativo "S-Plus" (Versão 4.5 Professional Release 2 for MS Windows, 1998, Copyright (C) Statistical Sciences, Inc. Copyright AT \& T). A descrição técnica deste modelo está apresentada no Apêndice 3 .

A figura 7 apresenta os dados de FC, em bpm, obtidos batimento a batimento, em tempo real, durante o TEFDD-D na potência $35 \mathrm{~W}$ de uma das voluntárias jovens estudadas (RTK). A figura 8 apresenta o conjunto de dados da FC, no intervalo de 120 a 300 s, ajustados pelo modelo SPM para a mesma voluntária. Verifica-se nesta figura que houve inclinação positiva e estatisticamente significante $(p<0,05)$, na qual foi considerado o LA para esta voluntária. A figura 9 apresenta o relatório de análise do ajuste do modelo SPM aos dados de FC da mesma voluntária e na figura 10 está 
representado o gráfico de probabilidade normal dos resíduos da série de dados de FC ajustados pelo modelo SPM.

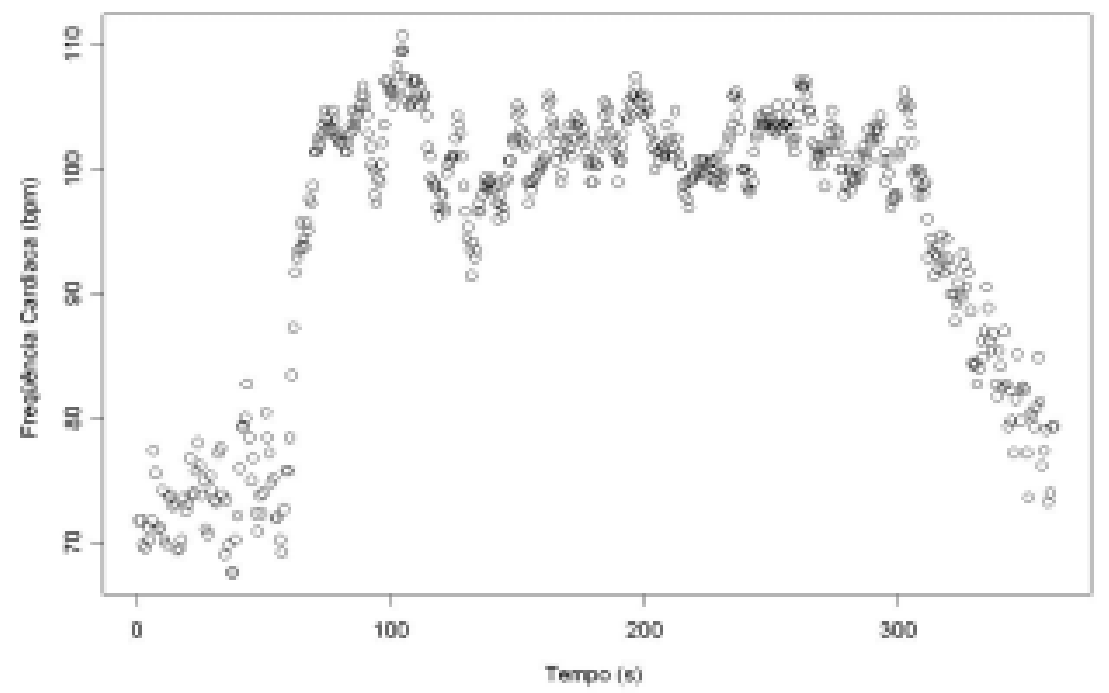

FIGURA 7. Resposta da freqüência cardíaca, em bpm, obtida batimento a batimento, em tempo real, durante 60 segundos em repouso, 240 segundos em exercício físico na potência de 35 Watts e 60 segundos em recuperação, de uma das voluntárias jovens estudadas (RTK). 


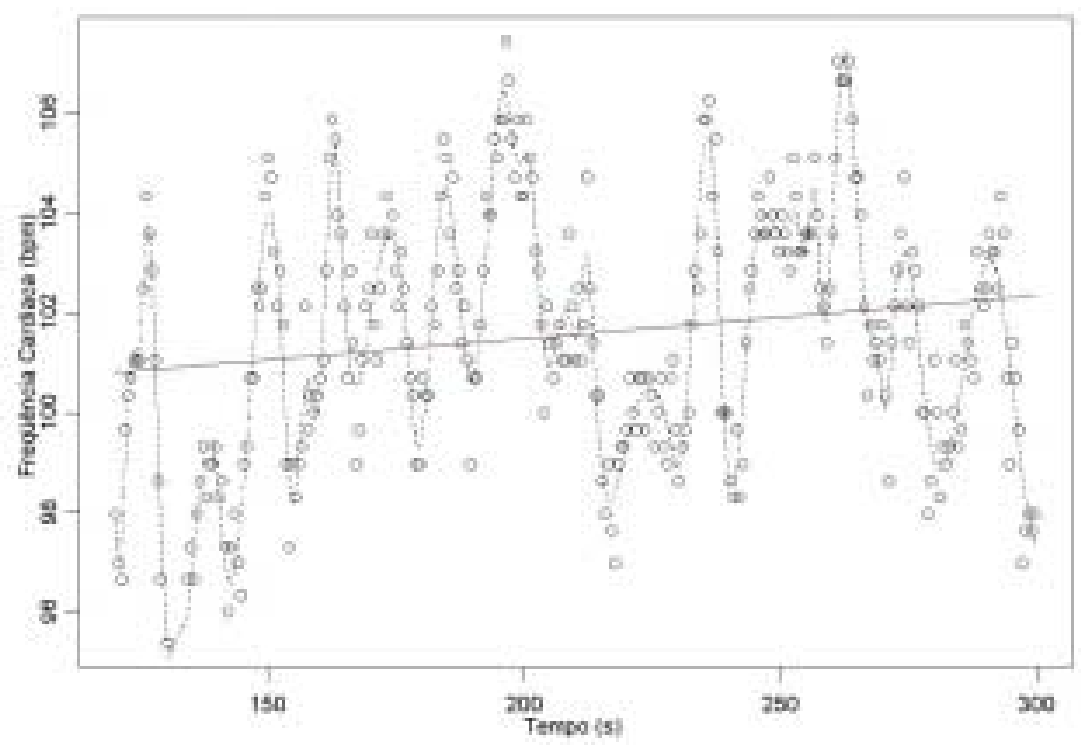

FIGURA 8. Ajuste do modelo matemático e estatístico semiparamétrico ao conjunto de dados de freqüência cardíaca no período de 120 a 300 segundos de exercício físico, mostrando tendências positivas de inclinação da reta, na potência de 35 Watts do teste de esforço físico dinâmico descontínuo do tipo degrau, realizado por uma das voluntárias jovens estudadas (RTK). 


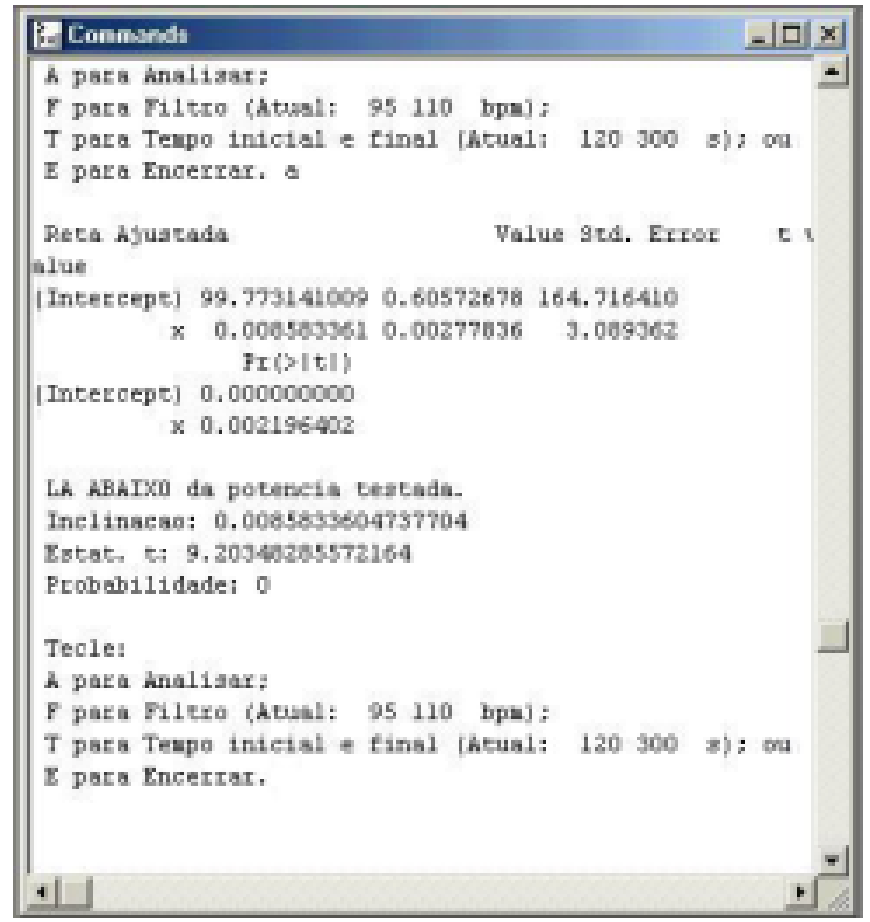

FIGURA 9. Relatório da análise do ajuste do modelo matemático e estatístico semiparamétrico, aos dados de freqüência cardíaca obtidos durante teste de esforço físico dinâmico descontínuo do tipo degrau na potência de 35 Watts, de uma das voluntárias jovens estudadas (RTK). 


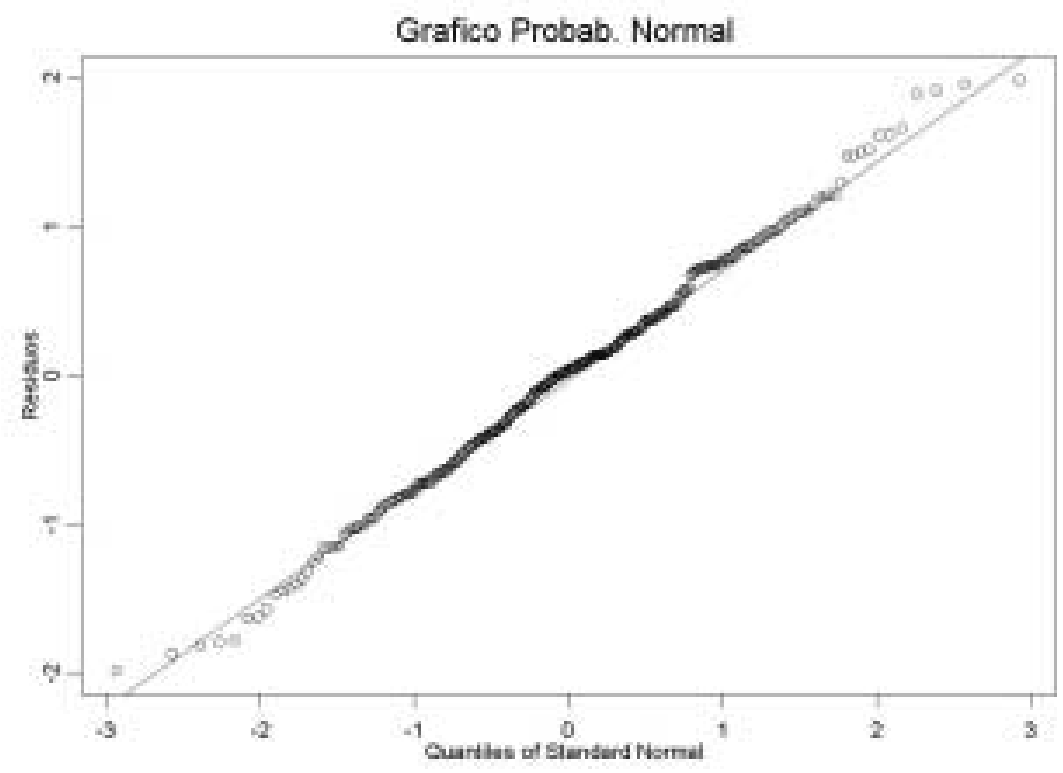

FIGURA 10. Gráfico de probabilidade normal dos resíduos da série de dados de freqüência cardíaca ajustados pelo modelo matemático e estatístico semiparamétrico, no período de 120 a 300 segundos de exercício físico, na potência de 35 Watts do teste de esforço físico dinâmico descontínuo do tipo degrau, de uma das voluntárias jovens estudadas (RTK).

$\mathrm{Na}$ potência do TEFDD-D em que foi observada a perda da estabilidade da FC, foram analisados os dados dos intervalos $R-R$ do mesmo período de tempo (120 a 300 s de exercício), por meio do cálculo dos índices de RMSSD. 


\subsubsection{Análise da variabilidade da freqüência cardíaca na determinação do limiar de anaerobiose por meio dos índices de RMSSD dos intervalos R-R}

Para determinação do LA pela análise da VFC utilizando-se o índice de RMSSD dos intervalos R-R foi aplicado o teste estatístico de Friedman, para comparar o valor obtido deste índice na condição de repouso sentado com os obtidos nas diferentes potências do TEFDD-D, utilizando-se para isso o aplicativo "GraphPad InStat for Windows Versão 3.0 1994-1999". A potência do TEFDD-D na qual foi detectada diferença estatisticamente significante $(p<0,05)$ em relação à condição de repouso sentada, foi considerada como o nível de esforço em que o grupo analisado atingiu o LA.

Para análise comparativa entre os 2 métodos de determinação do LA (modelo SPM $x$ VFC), foi utilizado os índices de RMSSD dos intervalos R-R (ms), obtidos para o conjunto de dados dos 2 métodos. 


\subsection{Metodologia Estatística}

Para se escolher que tipo de teste estatístico seria empregado (paramétrico ou não paramétrico) procedeu-se a uma avaliação quanto aos tipos de distribuição das variáveis estudadas em diferentes condições (figura 11). A análise de distribuição dos dados mostrou que os mesmos não possuíam distribuição normal. Portanto, foram utilizados testes estatísticos não paramétricos. Os resultados foram apresentados graficamente em "Boxplot" contendo os valores da mediana, 1ำ quartil (25\%), 3ำ quartil (75\%), valores máximos e mínimos, "outliers" e extremos, utilizando-se o aplicativo "STATISTICA for Windows, Release 5.5 StatSoft, Inc. (2000) e GraphPad InStat for Windows Versão 3.0 1994-1999".

A análise estatística de significância foi realizada a partir de testes não paramétricos: teste de Wilcoxon para amostras pareadas, teste de Kruskall-Wallis para amostras não pareadas e o teste de Friedman (ANOVA) para medidas repetidas, sendo os dois últimos seguido de técnicas de comparações múltiplas com o teste de Dunn, que mostra onde se deu a significância entre os dados, e análise de correlação de Spearman. Foram considerados níveis de significância de $\alpha=0,05$. 

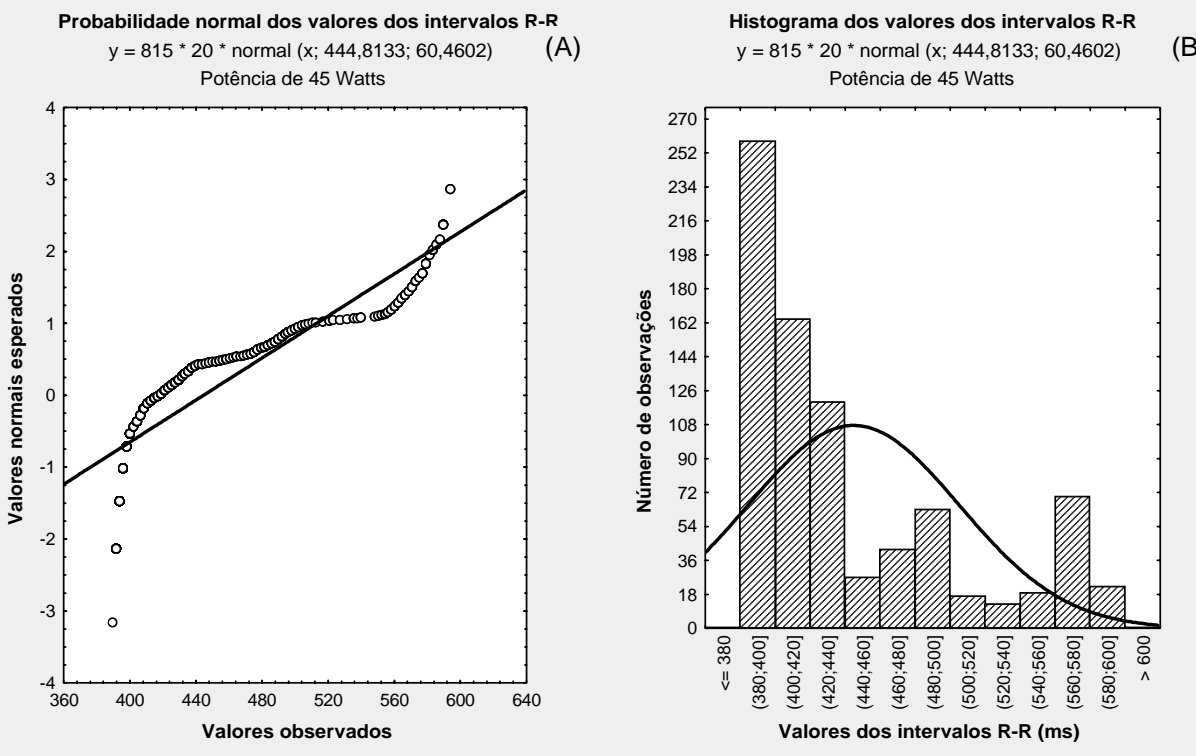

FIGURA 11. Análise dos valores absolutos dos intervalos $R-R$ para a potência de 45 Watts de uma das voluntárias pós-menopausa com reposição hormonal estudada quanto à distribuição dos dados. Em A, estão representados os valores observados em relação aos esperados e em $\mathrm{B}$, estão representados a Curva de Gauss e o Histograma do número de observações. 


\section{RESULTADOS}

\subsection{Idade, características antropométricas e clínicas das voluntárias estudadas}

Estão apresentados na tabela 1, em valores médios e respectivos desvios padrões (DP), os dados referentes à idade em anos, peso em Kilograma $(\mathrm{Kg})$, altura em metros $(\mathrm{m})$, índice de massa corporal (IMC) em Kilograma por metro quadrado $\left(\mathrm{Kg} / \mathrm{m}^{2}\right)$, pressão arterial sistólica (PAS) e diastólica (PAD) em milímetros de mercúrio (mmHg), FC em bpm, sendo que os três últimos foram obtidos em repouso, tempo do ciclo menstrual em dias, tempo de pós-menopausa e tempo de terapia de reposição hormonal, em anos, dos 3 grupos estudados.

Os dados apresentados nesta tabela mostram que:

- Quanto à idade (Jovens = $24 \pm 2,87$ anos; PMSRH = 57 \pm 5,28 anos; PMCRH $=55 \pm 5,40$ anos), foi observado diferença estatisticamente significante $(p<0,05)$ entre as voluntárias jovens e PMSRH e entre as jovens e PMCRH;

- Em relação ao peso corporal (Jovens $=58 \pm 6,60 \mathrm{Kg} ; \mathrm{PMSRH}=62 \pm$ 9,07 Kg; $\mathrm{PMCRH}=67 \pm 9,96 \mathrm{Kg}$ ), não encontramos diferenças estatisticamente significantes ( $p>0,05)$ entre os grupos; 
- Quanto à altura (Jovens $=1,62 \pm 0,05 \mathrm{~m}$; PMSRH $=1,57 \pm 0,06 \mathrm{~m}$; PMCRH $=1,55 \pm 0,05 \mathrm{~m})$, não foi observada diferenças estatisticamente significantes ( $p>0,05)$ entre os grupos;

- Os dados referentes ao IMC (Jovens $=22,18 \pm 2,52 \mathrm{Kg} / \mathrm{m}^{2} ; \mathrm{PMSRH}=$ $\left.25,07 \pm 3,46 \mathrm{Kg} / \mathrm{m}^{2} ; \mathrm{PMCRH}=27,64 \pm 3,57 \mathrm{Kg} / \mathrm{m}^{2}\right)$, mostraram diferenças estatisticamente significantes $(p<0,05)$ apenas entre as voluntárias jovens e PMCRH;

- Quanto aos valores de PAS e PAD (Jovens $=97 \pm 6,75$ e $65 \pm 5,27$ $\mathrm{mmHg} ; \mathrm{PMSRH}=123 \pm 14,49$ e $78 \pm 9,44 \mathrm{mmHg} ; \mathrm{PMCRH}=111 \pm$ 13,64 e $74 \pm 7,26 \mathrm{mmHg}$, respectivamente), foi observada diferença estatisticamente significante $(p<0,05)$ apenas entre as voluntárias jovens e PMSRH;

- Os valores de FC (Jovens $=73 \pm 9,31 \mathrm{bpm} ; \mathrm{PMSRH}=67 \pm 5,53 \mathrm{bpm}$; $\mathrm{PMCRH}=65 \pm 10,56 \mathrm{bpm})$ não mostraram diferenças estatisticamente significantes ( $p>0,05)$ entre os grupos;

- As voluntárias jovens apresentavam um ciclo menstrual de $29 \pm 1,63$ dias;

- $\mathrm{O}$ tempo de pós-menopausa (PMSRH $=9 \pm 5,72$ anos; $\mathrm{PMCRH}=7 \pm$ 4,72 anos) foi similar entre os grupos, não apresentando diferenças estatisticamente significantes $(p>0,05)$;

- O tempo de reposição hormonal das voluntárias PMCRH era de $6 \pm$ 4,35 anos.

$\mathrm{Na}$ tabela 2 estão apresentados os valores de referência e os valores obtidos da dosagem plasmática do hormônio folículo estimulante (FSH) em 
micro unidades internacionais por mililitro de sangue $(\mathrm{mUl} / \mathrm{ml})$ e do hormônio estradiol em picogramas por mililitro de sangue $(\mathrm{pg} / \mathrm{ml})$, realizada nas voluntárias PMSRH e PMCRH. Por esta tabela podemos observar, que os 2 grupos apresentam valores dentro da normalidade.

TABELA 1. Idade, características antropométricas e dados de freqüência cardíaca (FC), em bpm, de pressão arterial sistólica (PAS) e diastólica (PAD), em $\mathrm{mmHg}$, do tempo de ciclo menstrual, em dias, e tempo de pósmenopausa e de terapia de reposição hormonal (TRH), em anos, das voluntárias estudadas jovens, pós-menopausa sem reposição hormonal (PMSRH) e pós-menopausa com reposição hormonal (PMCRH).

\begin{tabular}{|c|c|c|c|}
\hline Características & $\begin{array}{c}\text { Jovens }(n=10) \\
x \pm D P\end{array}$ & $\begin{array}{c}\text { PMSRH }(n=13) \\
X \pm \text { DP }\end{array}$ & $\begin{array}{c}\text { PMCRH }(n=9) \\
X \pm D P\end{array}$ \\
\hline Idade (anos) & $24 \pm 2,87$ & $57 \pm 5,28^{*}$ & $55 \pm 5,40^{*}$ \\
\hline Peso (Kg) & $58 \pm 6,60$ & $62 \pm 9,07$ & $67 \pm 9,96$ \\
\hline Altura (m) & $1,62 \pm 0,05$ & $1,57 \pm 0,06$ & $1,55 \pm 0,05$ \\
\hline IMC $\left(\mathrm{Kg} / \mathrm{m}^{2}\right)$ & $22,18 \pm 2,52$ & $25,07 \pm 3,46$ & $27,64 \pm 3,57^{\star}$ \\
\hline PAS (mmHg) & $97 \pm 6,75$ & $123 \pm 14,49^{*}$ & $111 \pm 13,64$ \\
\hline PAD (mmHg) & $65 \pm 5,27$ & $78 \pm 9,44^{\star}$ & $74 \pm 7,26$ \\
\hline FC (bpm) & $73 \pm 9,31$ & $67 \pm 5,53$ & $65 \pm 10,56$ \\
\hline T de ciclo menstrual (dias) & $29 \pm 1,63$ & - & - \\
\hline T de pós-menopausa (anos) & - & $9 \pm 5,72$ & $7 \pm 4,72$ \\
\hline T de TRH (anos) & - & - & $6 \pm 4,35$ \\
\hline
\end{tabular}


TABELA 2. Dados dos valores de referência dos níveis hormonais e dos valores observados nas voluntárias pós-menopausa com reposição hormonal $(\mathrm{PMCRH})$ e pós-menopausa sem reposição hormonal (PMSRH).

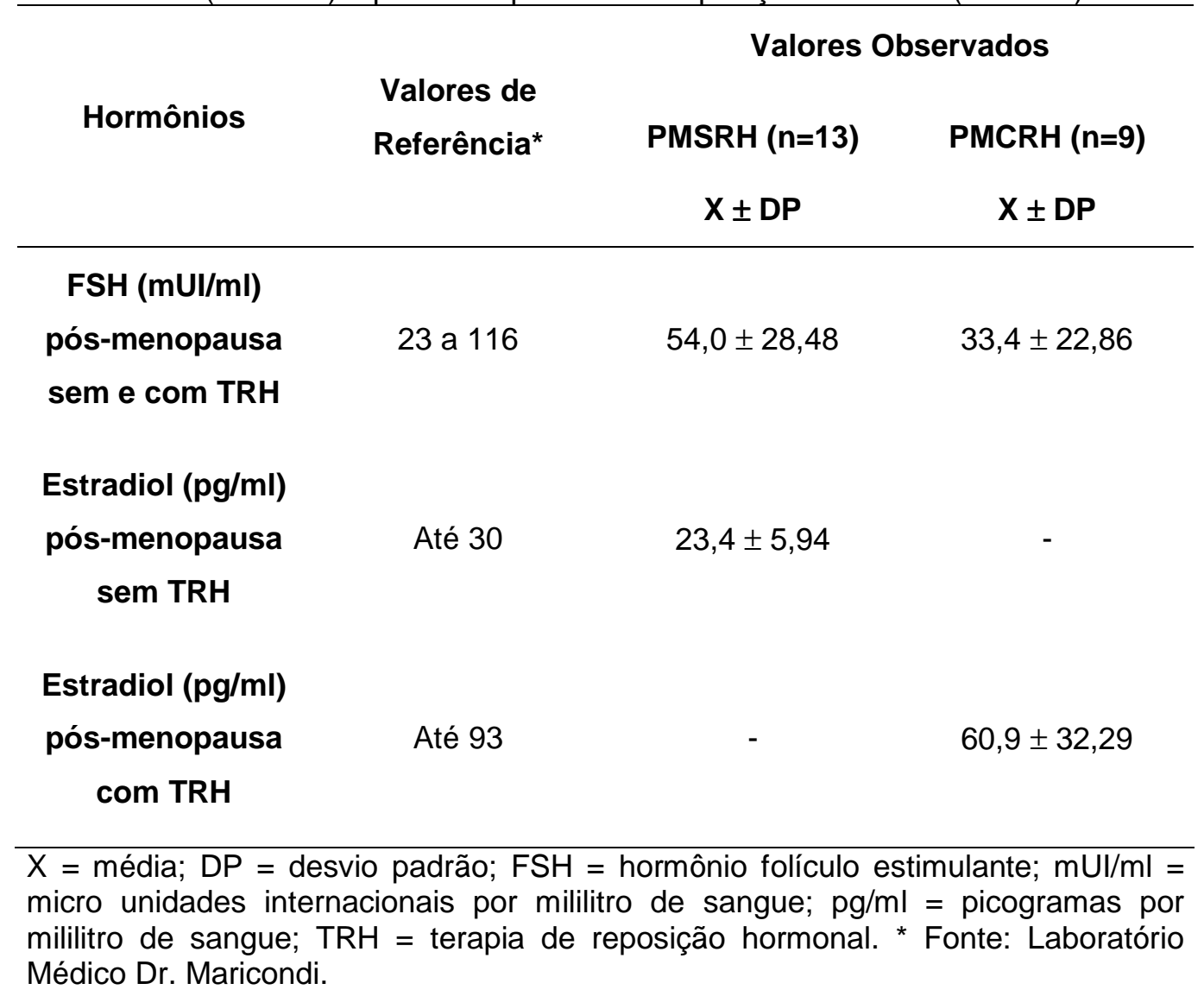




\subsection{Análise descritiva da freqüência cardíaca e de sua variabilidade}

Nas figuras 12, 13 e 14, está ilustrado o comportamento da resposta da FC e de sua variabilidade, obtida batimento a batimento, em tempo real, na condição de repouso, nas posições supina e sentada, por um período de 360 s em cada posição e durante cada potência do TEFDD-D por um período de $360 \mathrm{~s}$, sendo $60 \mathrm{~s}$ pré-esforço, $240 \mathrm{~s}$ de exercício e $60 \mathrm{~s}$ de recuperação, de uma voluntária jovem (CCESO), uma PMSRH (DBS) e uma PMCRH (ESV), respectivamente.

Podemos observar visualmente nestas figuras que o grupo PMCRH apresenta valores absolutos de FC em repouso, nas posições supina e sentada, menores em relação aos grupos de voluntárias jovens e PMSRH.

Verifica-se nestas figuras que no início do exercício físico, o padrão de resposta da FC é caracterizado por uma elevação rápida. Após este período inicial, ocorre uma redução da $\mathrm{FC}$, mais pronunciada nas voluntárias jovens e PMCRH. Podemos também observar que nas potências mais baixas do teste de esforço físico, a FC tende a uma estabilização, enquanto que nas potências mais elevadas observa-se um incremento lento da FC.

Em relação à VFC, observa-se uma diminuição da mesma com a transição do repouso para o exercício físico, sendo que esta diminuição acentua-se com o incremento de potências durante o TEFDD-D. Além disso, verifica-se que as voluntárias do grupo jovem apresentam maior VFC em relação aos grupos PMSRH e PMCRH, tanto em repouso como em exercício físico. 


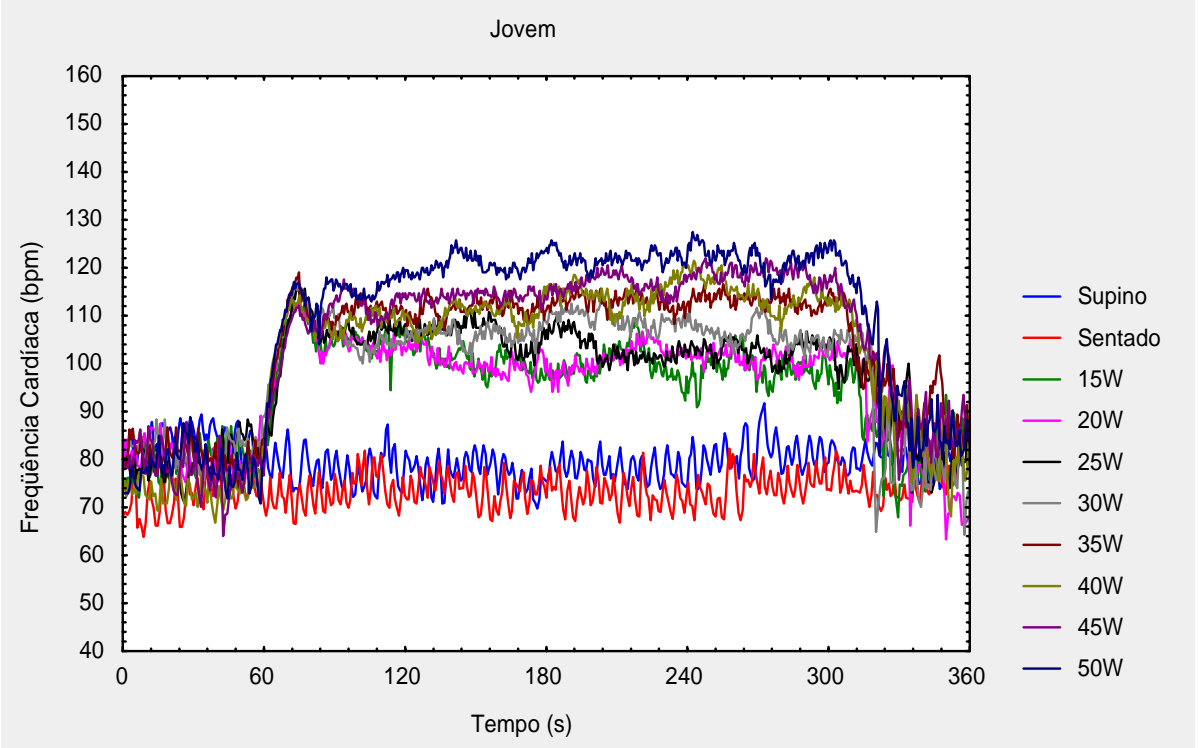

FIGURA 12. Resposta da freqüência cardíaca em batimentos por minuto (bpm), batimento a batimento, em tempo real, durante 360 segundos (s) em repouso, nas posições supina e sentada e durante $60 \mathrm{~s}$ em repouso pré-esforço, $240 \mathrm{~s}$ de esforço físico dinâmico com potências progressivas de 15, 20, 25, 30, 35, 40, 45 e 50 Watts (W) e $60 \mathrm{~s}$ após o esforço, de uma das voluntárias jovens estudada (CCESO). 


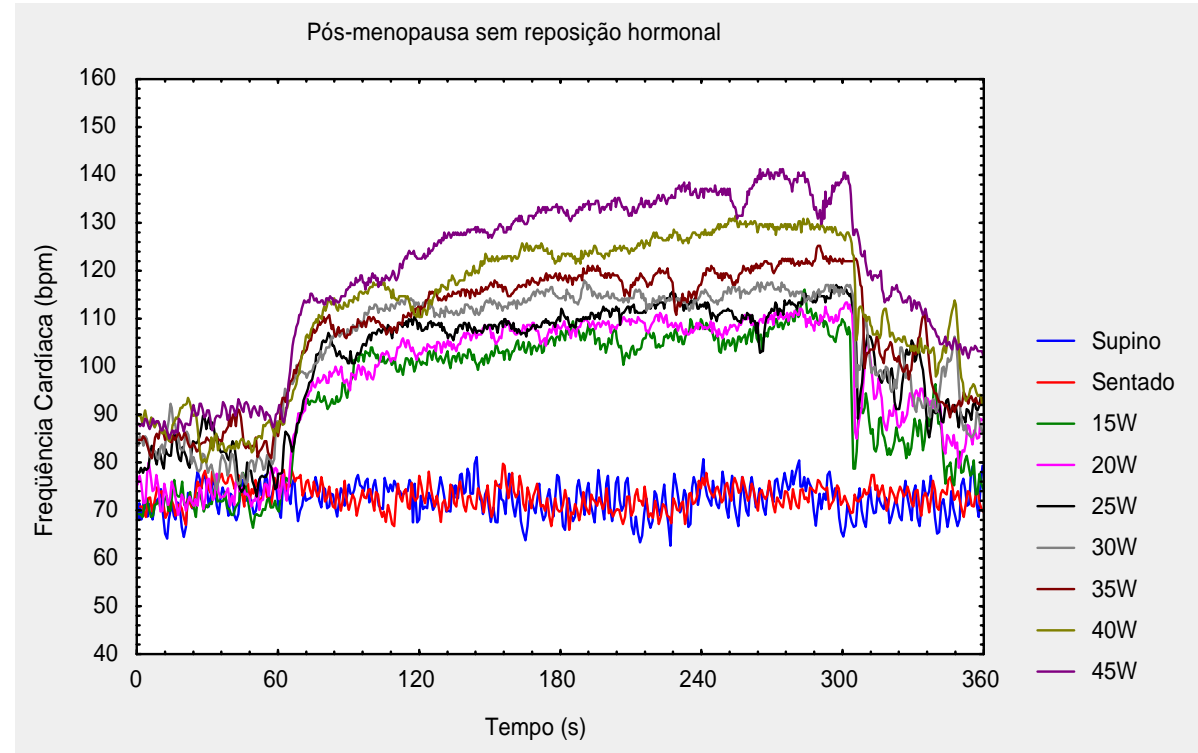

FIGURA 13. Resposta da freqüência cardíaca em batimentos por minuto (bpm), batimento a batimento, em tempo real, durante 360 segundos (s) em repouso, nas posições supina e sentada e durante $60 \mathrm{~s}$ em repouso pré-esforço, $240 \mathrm{~s}$ de esforço físico dinâmico com potências progressivas de 15, 20, 25, 30, 35, 40 e 45 Watts (W) e 60 s após o esforço, de uma das voluntárias pós-menopausa sem reposição hormonal estudada (DBS). 


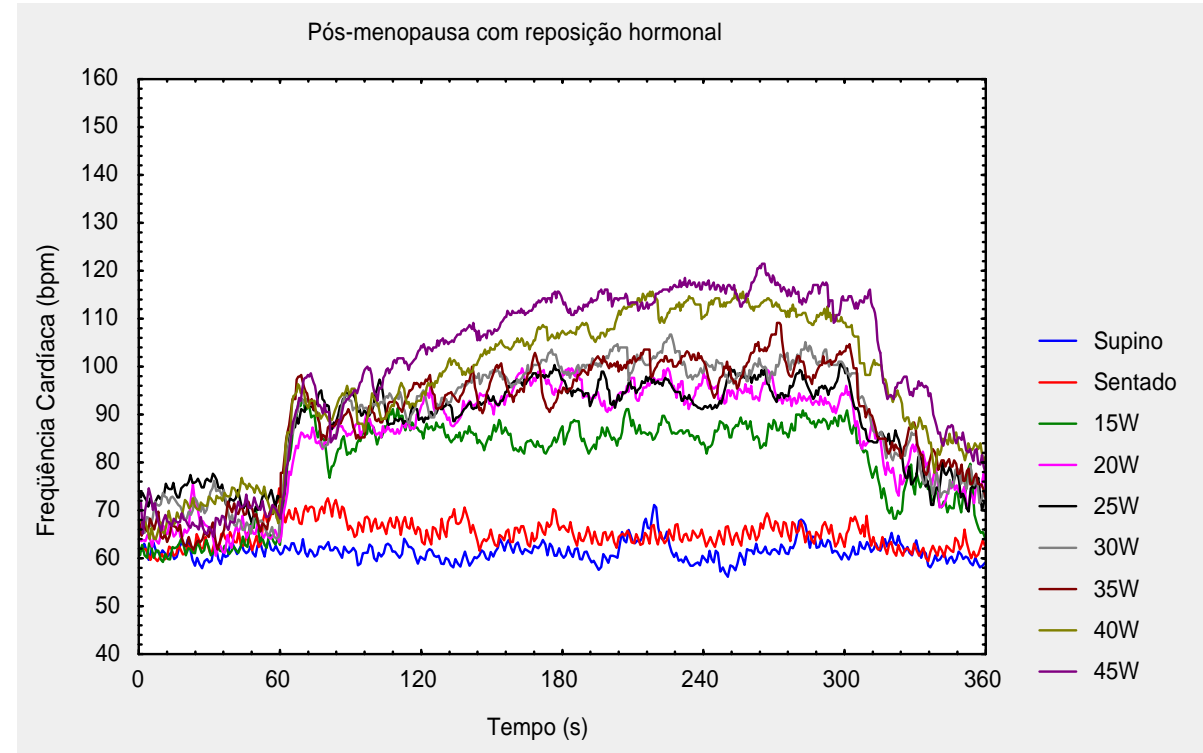

FIGURA 14. Resposta da freqüência cardíaca, em batimentos por minuto (bpm), batimento a batimento, em tempo real, durante 360 segundos (s) em repouso, nas posições supina e sentada e durante $60 \mathrm{~s}$ em repouso pré-esforço, $240 \mathrm{~s}$ de esforço físico dinâmico com potências progressivas de 15, 20, 25, 30, 35, 40 e 45 Watts (W) e 60 s após o esforço, de uma das voluntárias pós-menopausa com reposição hormonal estudada (ESV). 


\subsection{Análise da freqüência cardíaca e de sua variabilidade durante o repouso}

Nas figuras 15 e 16, estão apresentados os resultados de FC, em bpm, em valores médios, e dos índices de RMSSD dos intervalos R-R, em ms, respectivamente, calculados para a condição de repouso, nas posições supina e sentado.

Verifica-se na figura 15 que os valores em mediana da FC em repouso na posição supina foram inferiores aos da posição sentada, sendo que apenas os grupos PMSRH e PMCRH apresentaram diferenças estatisticamente significantes entre as posições $(p<0,05)$. Observa-se ainda nesta figura, que tanto na posição supina quanto na posição sentada, o grupo PMCRH apresentou valores inferiores de FC em relação aos outros 2 grupos, porém sem diferença estatisticamente significante $(p>0,05)$.

$\mathrm{Na}$ figura 16, verifica-se que as voluntárias jovens e $\mathrm{PMCRH}$ não apresentaram diferenças estatisticamente significantes $(p>0,05)$ dos índices de RMSSD dos intervalos R-R, em ms, entre as posições supina e sentada. Já as voluntárias PMSRH apresentaram valores superiores deste índice na posição supina em relação à sentada, com significância estatística $(p<0,05)$. $\mathrm{Na}$ comparação intergrupos deste índice, verifica-se que as voluntárias jovens apresentaram os maiores valores em repouso nas 2 posições, apresentando significância estatística $(p<0,05)$ em relação às voluntárias PMSRH na posição sentada e em relação às voluntárias PMCRH na posição supina. 


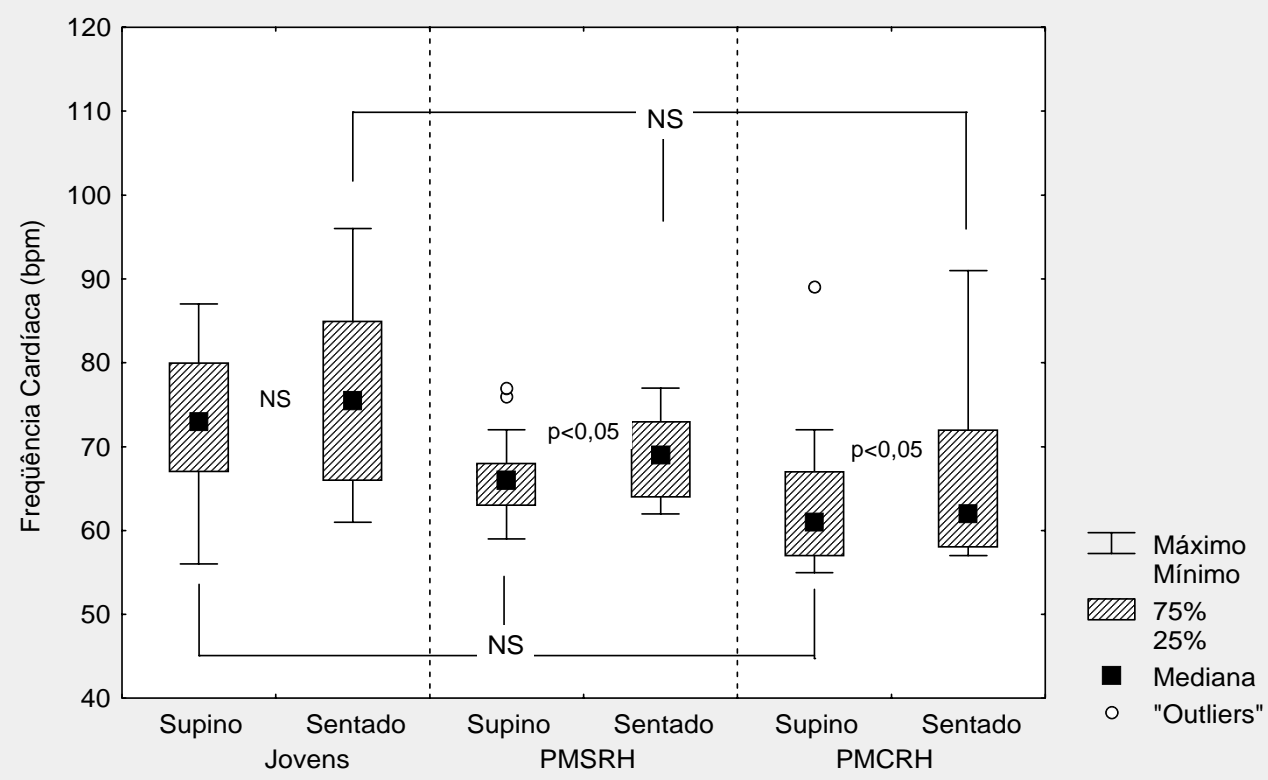

FIGURA 15. Valores de freqüência cardíaca, em batimentos por minuto (bpm), para o grupo de voluntárias jovens $(n=10)$, pós-menopausa sem reposição hormonal (PMSRH) $(n=13)$ e pós-menopausa com reposição hormonal $(\mathrm{PMCRH})(\mathrm{n}=9)$, durante o repouso, nas posições supina e sentada. Nível de significância de $\alpha=0,05$. 


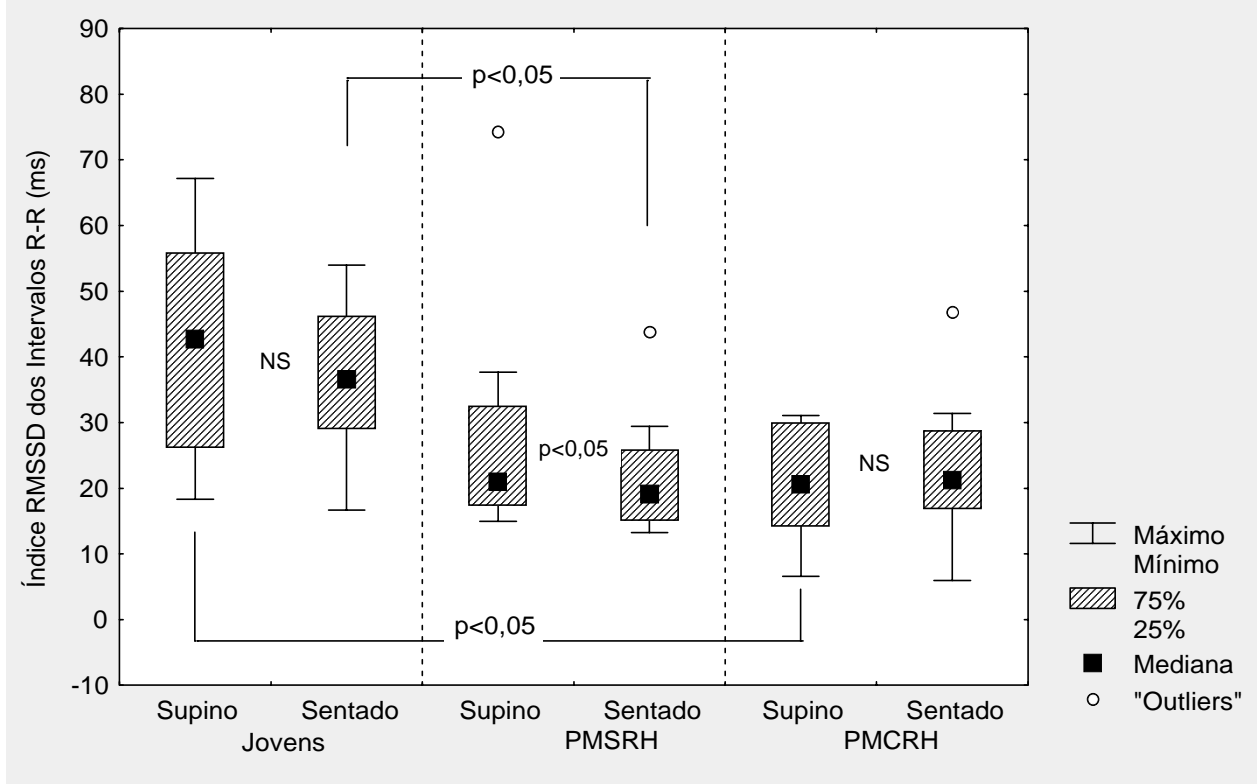

FIGURA 16. Valores dos índices de RMSSD dos intervalos R-R, em milissegundos (ms), para o grupo de voluntárias jovens $(n=10)$, pós-menopausa sem reposição hormonal (PMSRH) $(n=13)$ e pós-menopausa com reposição hormonal (PMCRH) $(n=9)$, durante o repouso, nas posições supina e sentada. Nível de significância de $\alpha=0,05$. 


\subsection{Análise da variação da freqüência cardíaca e do tempo na fase inicial do exercício físico}

Nas figuras de 17 a 23 estão apresentados em A, os dados referentes ao tempo de variação da $\mathrm{FC}$, em s; em $\mathrm{B}$, os valores de variação da $\mathrm{FC}$, em bpm, na fase inicial do exercício, nas potências de 15, 20, 25, 30, 35, 40 e $45 \mathrm{~W}$ do TEFDD-D, respectivamente, realizadas pelas voluntárias jovens, PMSRH e PMCRH.

Podemos observar nestas figuras que em $\mathrm{A}$, o tempo de variação da FC foi similar entre os grupos, em todas as potências analisadas, não apresentando diferenças estatisticamente significantes $(p>0,05)$. Em B, verifica-se que as voluntárias PMSRH apresentaram os menores valores de variação da FC em todos os níveis de esforço estudados, porém com significância estatística $(p<0,05)$ apenas em relação às voluntárias jovens, nas potências de 20, 25, 35 e $45 \mathrm{~W}$.

Verifica-se ainda, que enquanto os dados relacionados ao tempo de variação da FC das voluntárias jovens apresentam uma menor distância entre o $1^{\circ}$ e $\circ 3^{\circ}$ quartil, os dados de variação da FC apresentam uma maior distância entre os quartis, comparativamente aos outros 2 grupos. 


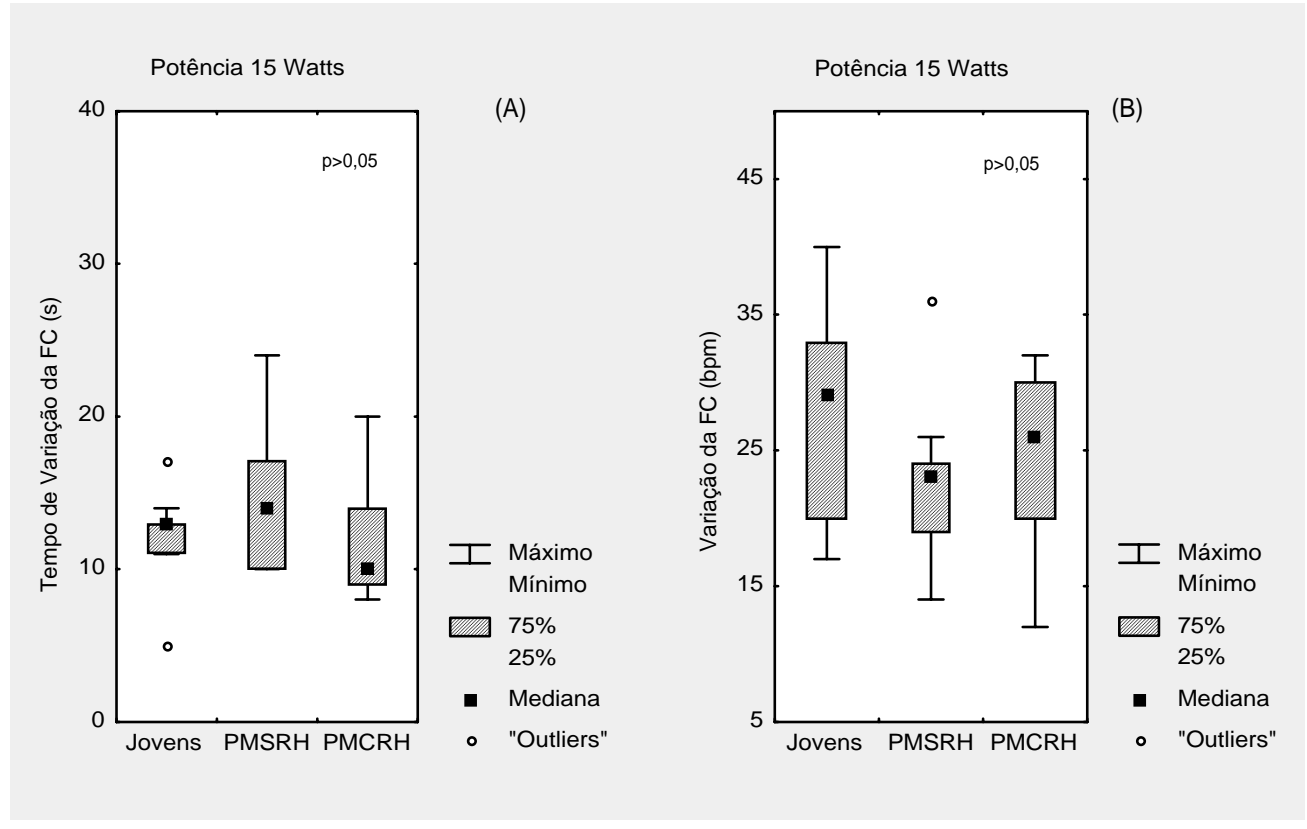

FIGURA 17. Em A, valores do tempo de variação da freqüência cardíaca, em segundos (s) e em $B$, valores de variação da freqüência cardíaca, em batimentos por minuto (bpm), na potência de 15 Watts do teste de esforço físico dinâmico descontínuo do tipo degrau, para o grupo de voluntárias jovens $(n=10)$, pós-menopausa sem reposição hormonal $(P M S R H) \quad(n=13)$ e pós-menopausa com reposição hormonal $(P M C R H)(n=9)$. Nível de significância de $\alpha=0,05$. 

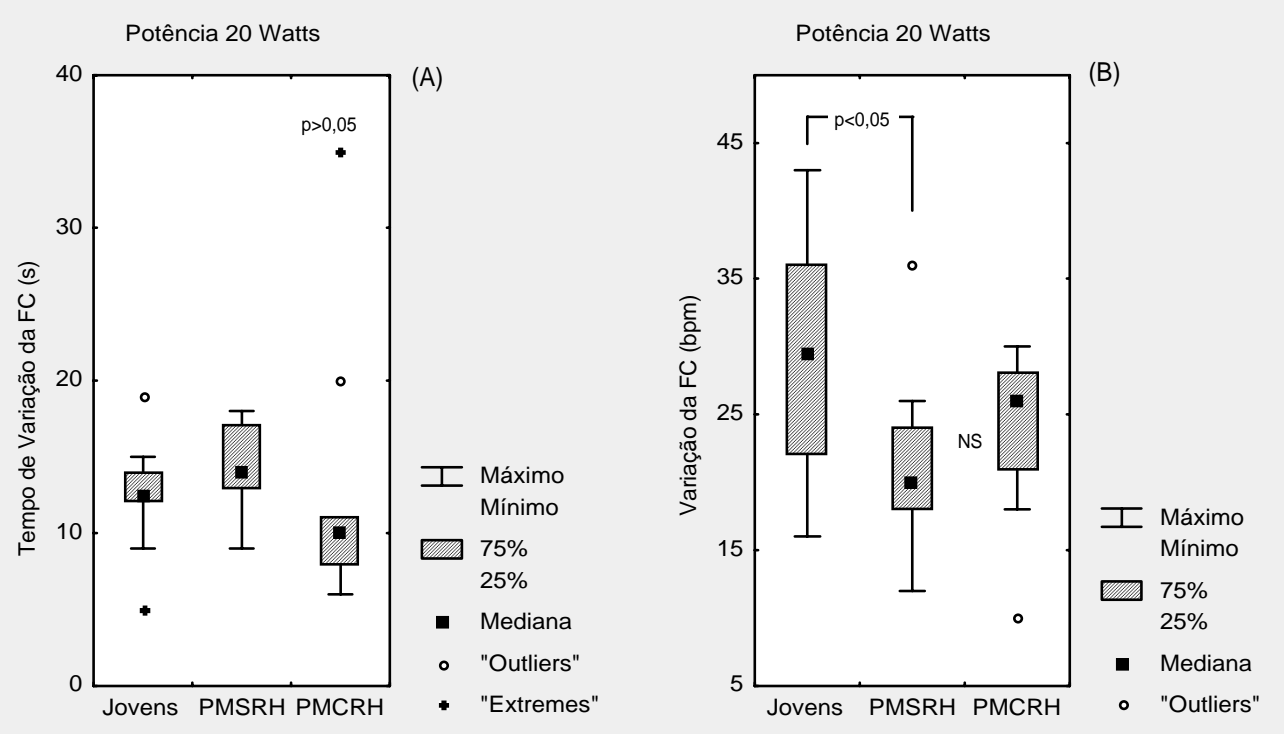

FIGURA 18. Em A, valores do tempo de variação da freqüência cardíaca, em segundos (s) e em $B$, valores de variação da freqüência cardíaca, em batimentos por minuto (bpm), na potência de 20 Watts do teste de esforço físico dinâmico descontínuo do tipo degrau, para o grupo de voluntárias jovens $(n=10)$, pós-menopausa sem reposição hormonal $(P M S R H) \quad(n=13)$ e pós-menopausa com reposição hormonal $(P M C R H)(n=9)$. Nível de significância de $\alpha=0,05$. 


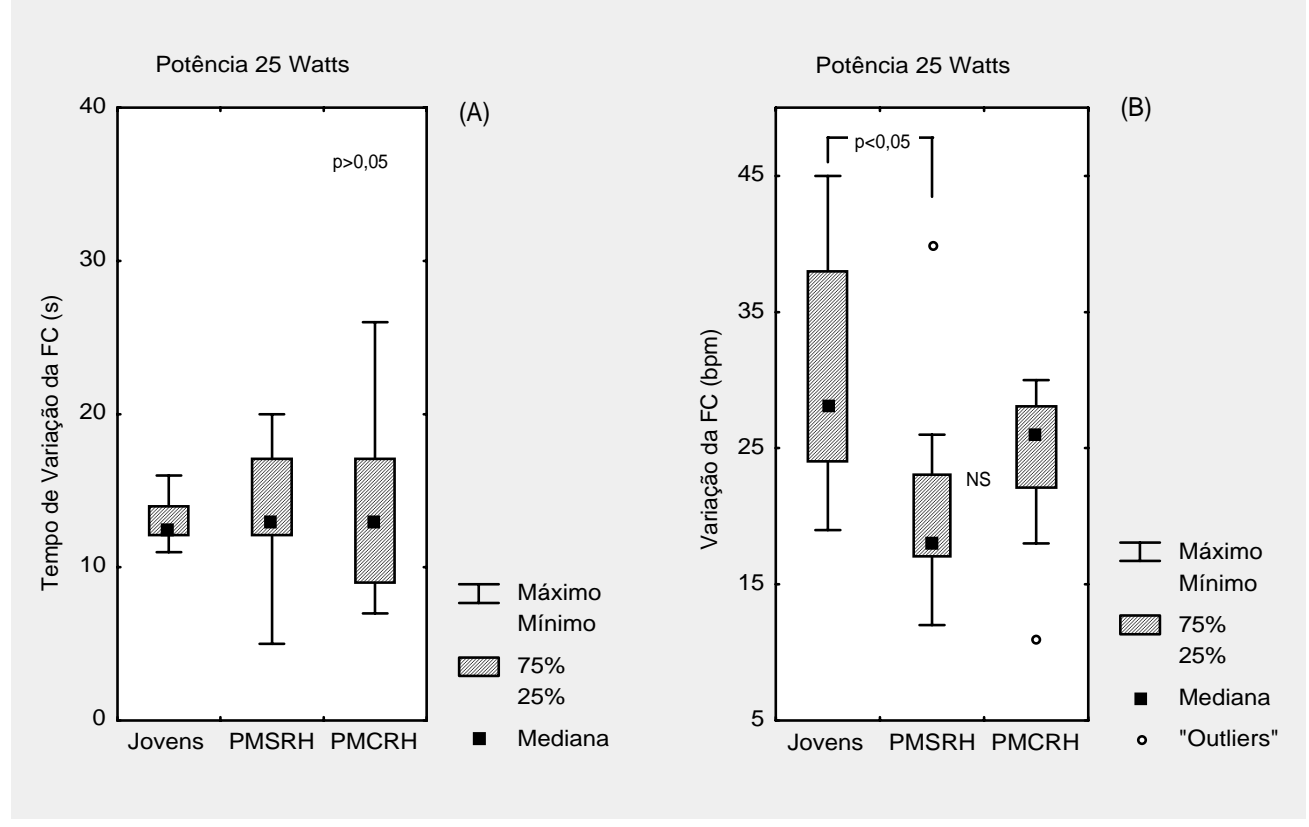

FIGURA 19. Em A, valores do tempo de variação da freqüência cardíaca, em segundos (s) e em B, valores de variação da freqüência cardíaca, em batimentos por minuto (bpm), na potência de 25 Watts do teste de esforço físico dinâmico descontínuo do tipo degrau, para o grupo de voluntárias jovens $(n=10)$, pós-menopausa sem reposição hormonal (PMSRH) $(n=13)$ e pós-menopausa com reposição hormonal $(\mathrm{PMCRH})(\mathrm{n}=9)$. Nível de significância de $\alpha=0,05$. 

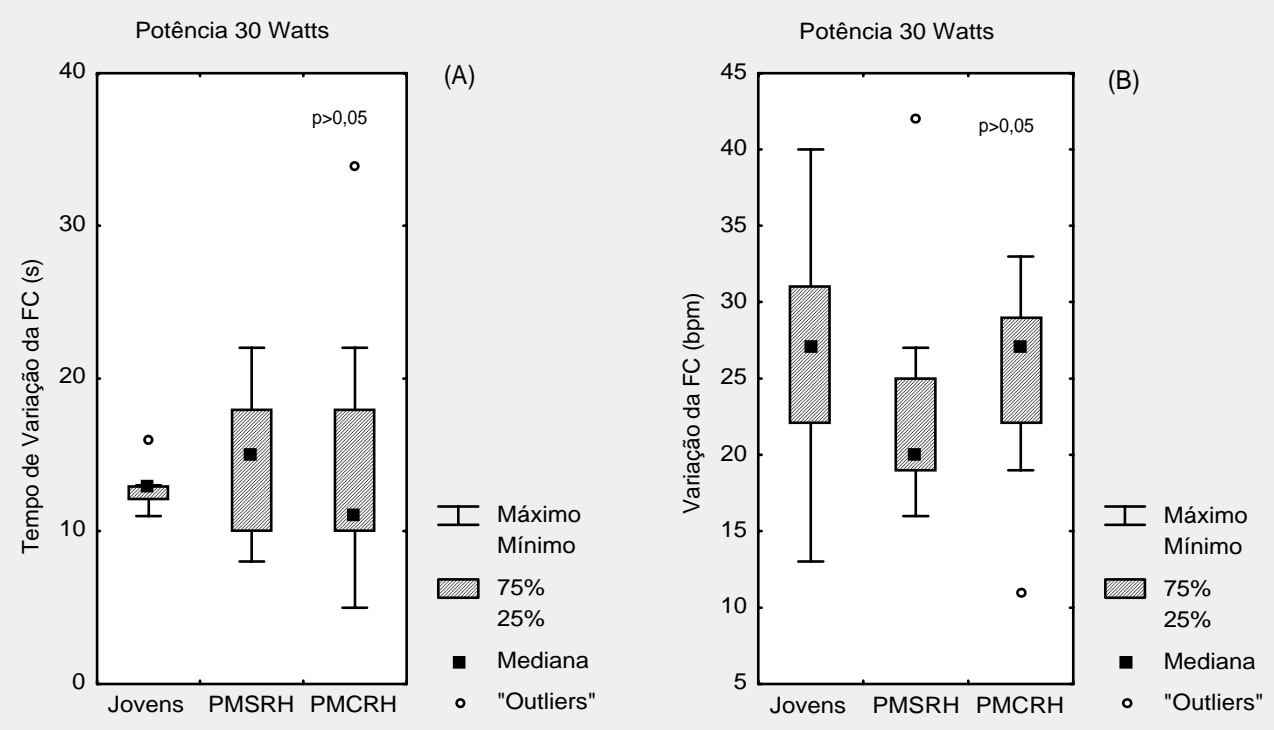

FIGURA 20. Em A, valores do tempo de variação da freqüência cardíaca, em segundos (s) e em $B$, valores de variação da freqüência cardíaca, em batimentos por minuto (bpm), na potência de 30 Watts do teste de esforço físico dinâmico descontínuo do tipo degrau, para o grupo de voluntárias jovens $(n=10)$, pós-menopausa sem reposição hormonal $(P M S R H) \quad(n=13)$ e pós-menopausa com reposição hormonal (PMCRH) ( $n=9)$. Nível de significância de $\alpha=0,05$. 


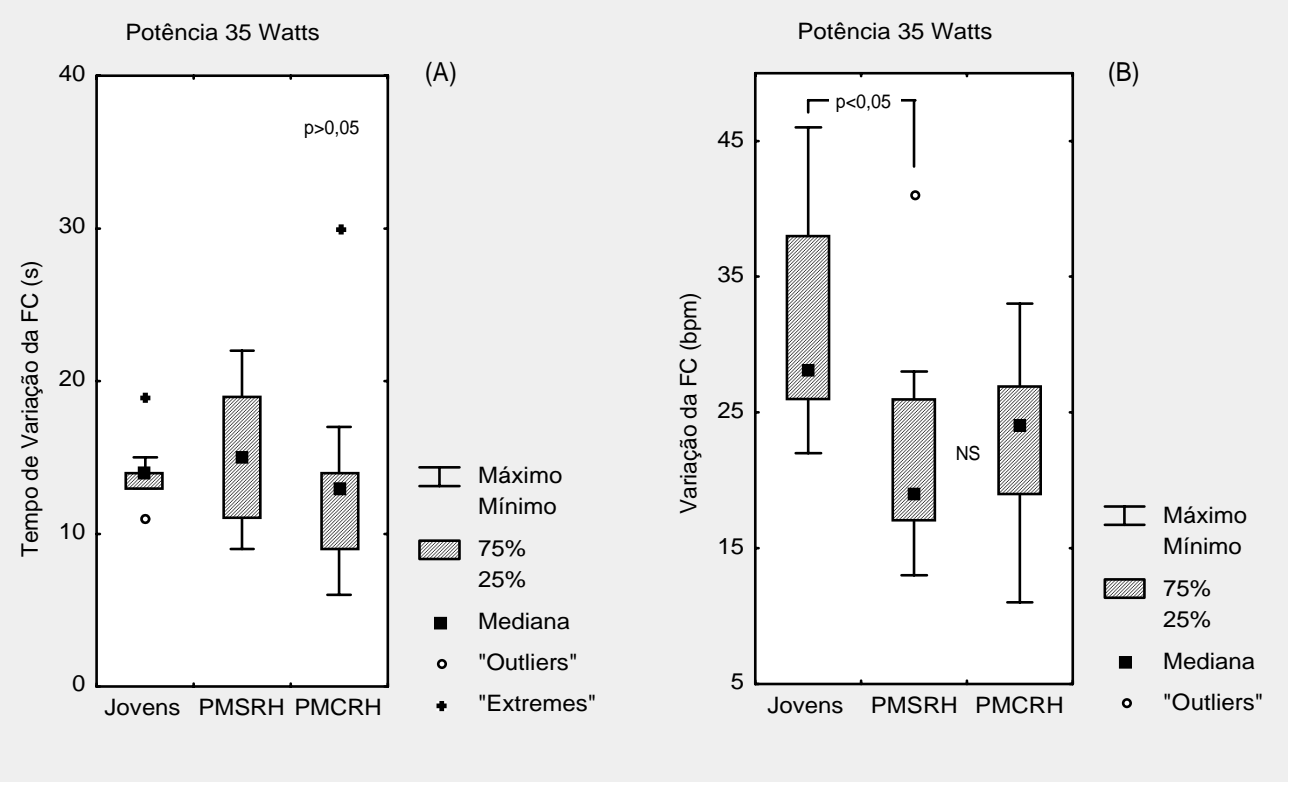

FIGURA 21. Em A, valores do tempo de variação da freqüência cardíaca, em segundos (s) e em $B$, valores de variação da freqüência cardíaca, em batimentos por minuto (bpm), na potência de 35 Watts do teste de esforço físico dinâmico descontínuo do tipo degrau, para o grupo de voluntárias jovens $(n=10)$, pós-menopausa sem reposição hormonal $(P M S R H) \quad(n=13)$ e pós-menopausa com reposição hormonal $(P M C R H)(n=9)$. Nível de significância de $\alpha=0,05$. 


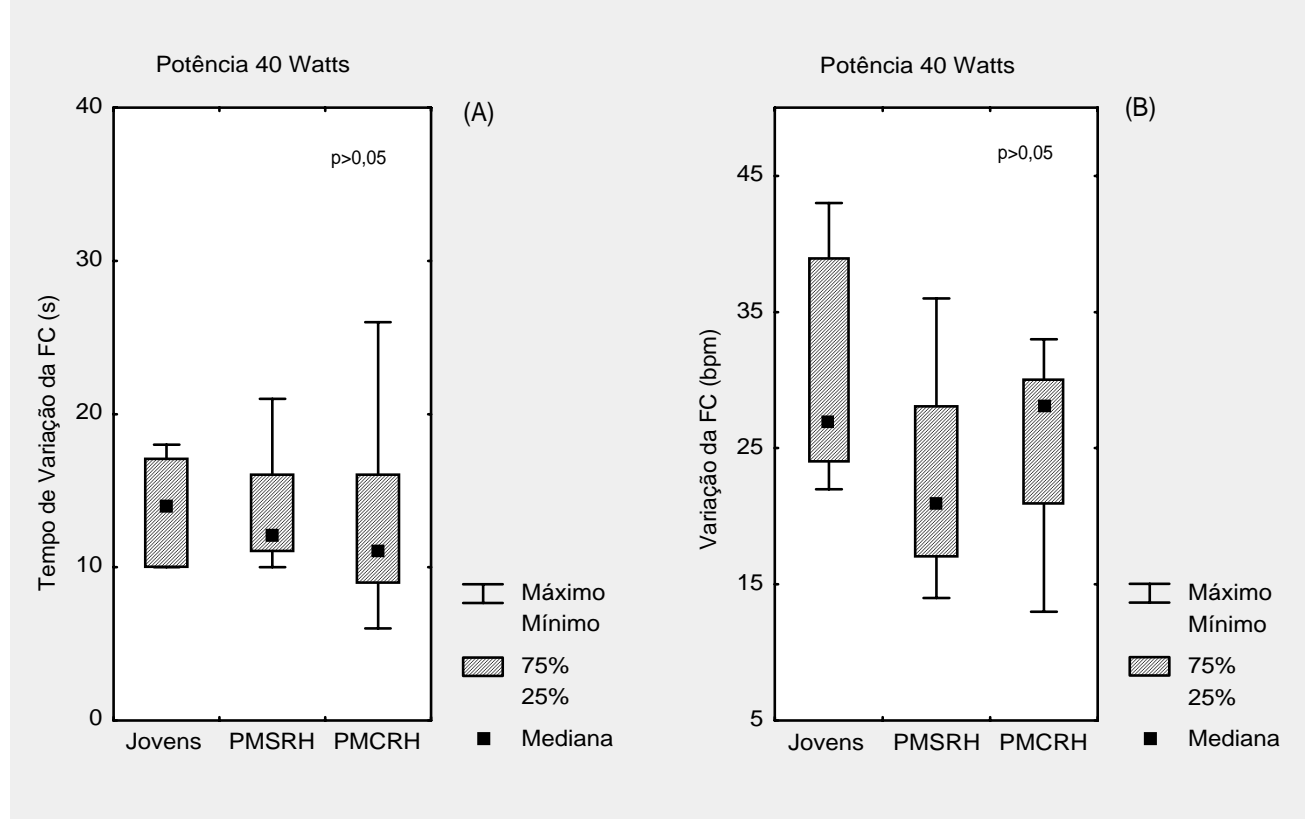

FIGURA 22. Em A, valores do tempo de variação da freqüência cardíaca, em segundos (s) e em $B$, valores de variação da freqüência cardíaca, em batimentos por minuto (bpm), na potência de 40 Watts do teste de esforço físico dinâmico descontínuo do tipo degrau, para o grupo de voluntárias jovens $(n=10)$, pós-menopausa sem reposição hormonal (PMSRH) $(n=11)$ e pós-menopausa com reposição hormonal $(\mathrm{PMCRH})(\mathrm{n}=8)$. Nível de significância de $\alpha=0,05$. 

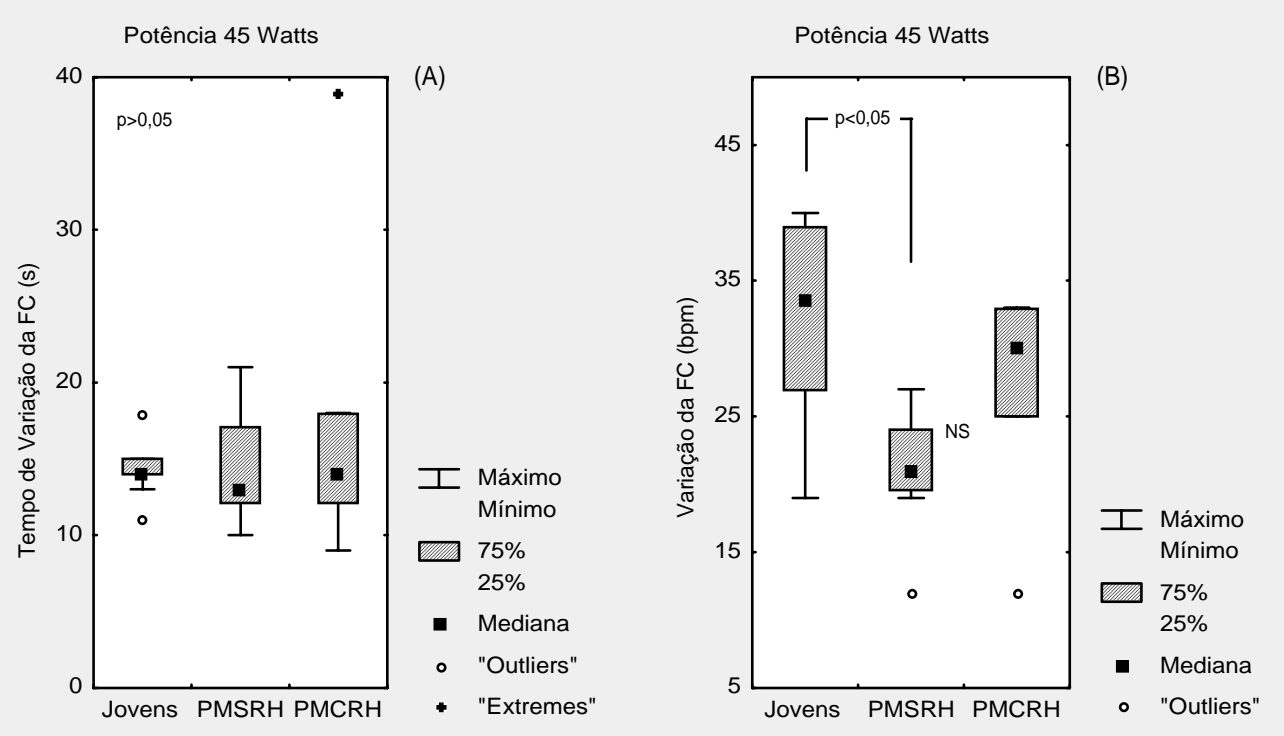

FIGURA 23. Em A, valores do tempo de variação da freqüência cardíaca, em segundos (s) e em $B$, valores de variação da freqüência cardíaca, em batimentos por minuto (bpm), na potência de 45 Watts do teste de esforço físico dinâmico descontínuo do tipo degrau, para o grupo de voluntárias jovens $(n=10)$, pós-menopausa sem reposição hormonal $(\mathrm{PMSRH}) \quad(\mathrm{n}=8)$ e pós-menopausa com reposição hormonal $(P M C R H)(n=7)$. Nível de significância de $\alpha=0,05$. 


\subsection{Análise da resposta da freqüência cardíaca e de sua variabilidade durante o teste de esforço físico dinâmico descontínuo do tipo degrau}

As figuras 24 a 26, mostram em A, os valores de FC, em bpm, e em B, dos índices de RMSSD dos intervalos R-R, em ms, calculados para a condição de repouso, na posição sentada, e para cada potência do TEFDDD. Foram consideradas para a análise as potências de 15 a $50 \mathrm{~W}$, realizadas em comum pelas voluntárias jovens e as potências de 15 a 35 W, realizadas em comum pelas voluntárias do grupo PMSRH e PMCRH.

Podemos observar nestas figuras que em relação ao repouso na posição sentada, para todos os níveis de esforço estudados, ocorre um aumento da FC (bpm) nos 3 grupos estudados, porém esse aumento só atingiu significância estatística $(p<0,05)$ a partir da potência de $30 \mathrm{~W}$ para 0 grupo de voluntárias jovens, 20 W para o grupo de voluntárias PMSRH e 25 W para o grupo de voluntárias $\mathrm{PMCRH}$, bem como uma diminuição dos valores dos índices de RMSSD dos intervalos R-R (ms), atingindo significância estatística $(p<0,05)$ a partir da potência de $30 \mathrm{~W}$ para as voluntárias jovens e $25 \mathrm{~W}$ para os grupos de voluntárias PMSRH e PMCRH.

Verifica-se ainda, que a diminuição dos valores dos índices de RMSSD dos intervalos R-R na transição do repouso para as potências do TEFDD-D, foi maior para o grupo de voluntárias jovens em relação aos demais grupos. 


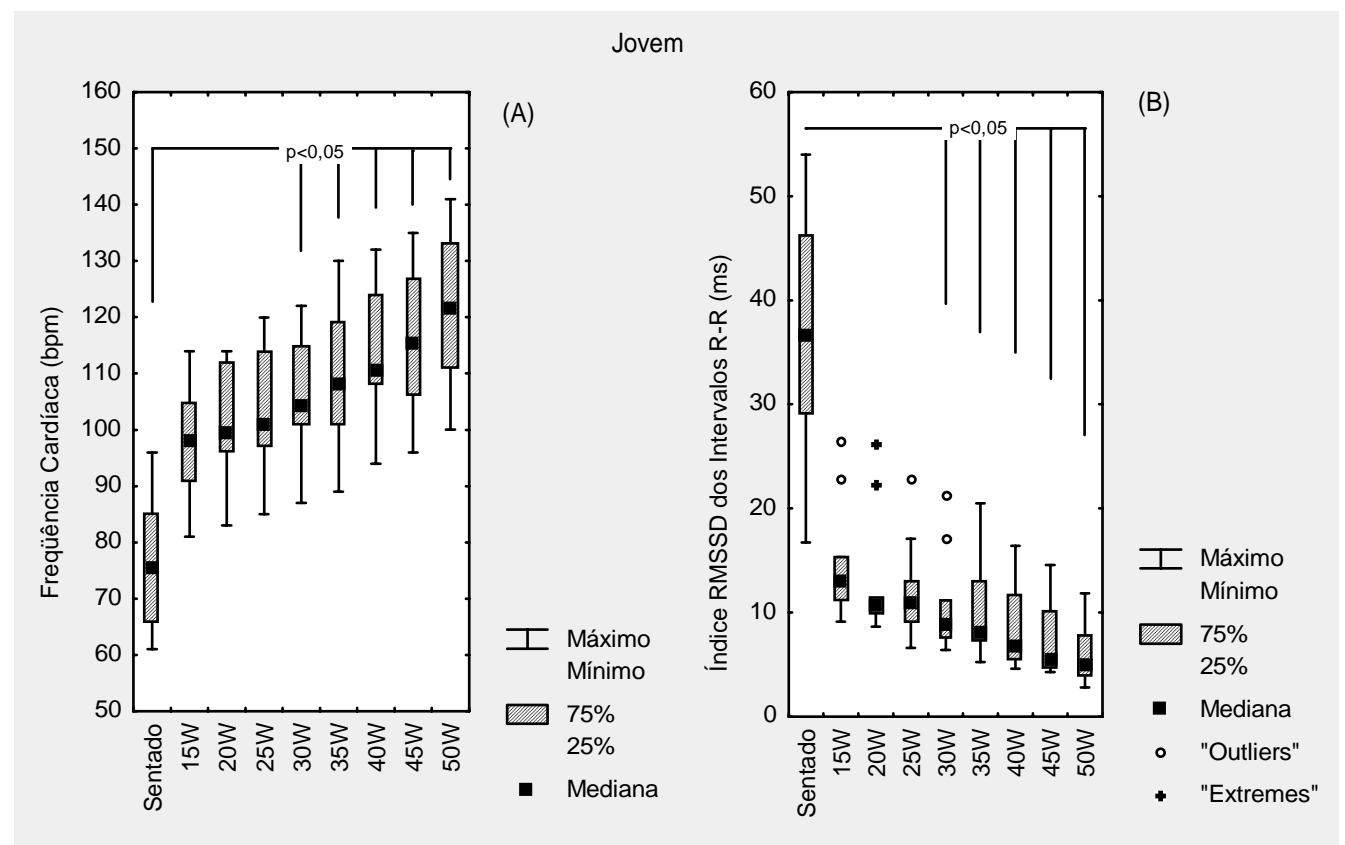

FIGURA 24. Em A, valores de freqüência cardíaca, em batimentos por minuto (bpm) e em B, dos índices de RMSSD dos intervalos R-R, em milissegundos (ms), para o grupo de voluntárias jovens $(n=10)$, na condição de repouso na posição sentada e durante as potências de 15 a 50 Watts $(\mathrm{W})$ do teste de esforço físico dinâmico descontínuo do tipo degrau. Nível de significância de $\alpha=0,05$. 

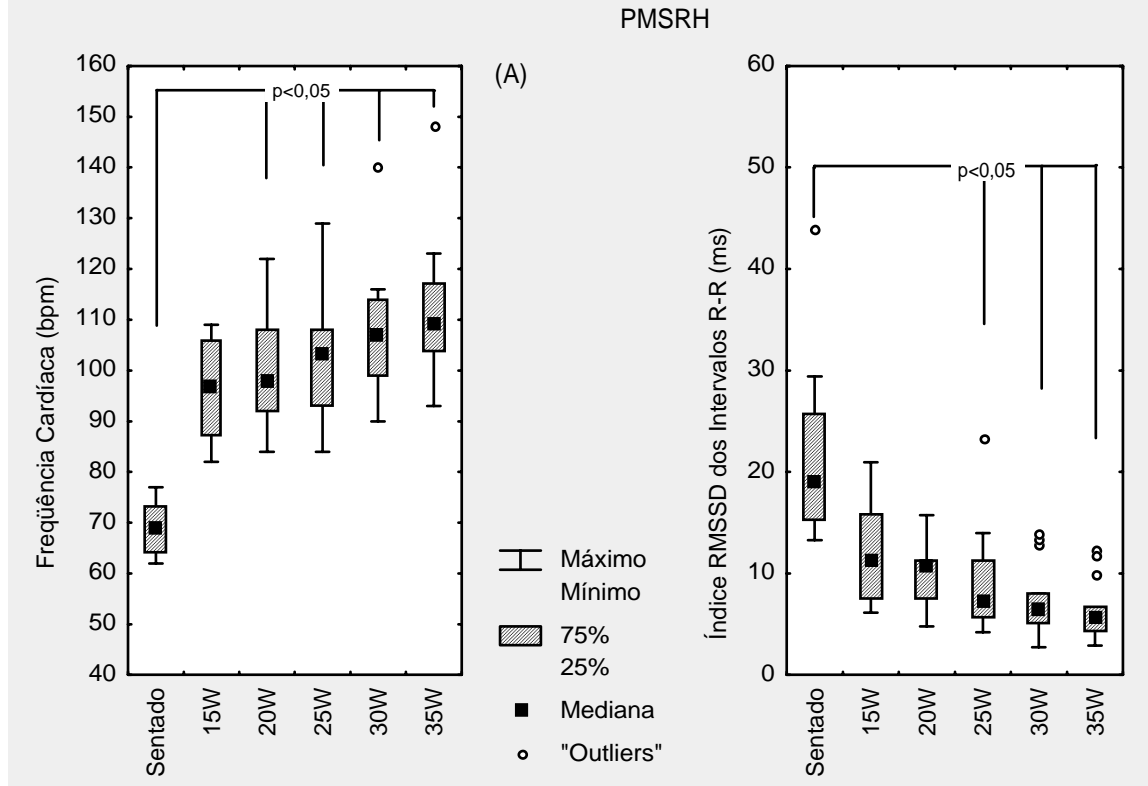

(B)

FIGURA 25. Em A, valores de freqüência cardíaca, em batimentos por minuto (bpm) e em B, dos índices de RMSSD dos intervalos R-R, em milissegundos (ms), para o grupo pós-menopausa sem reposição hormonal $(P M S R H)(n=13)$, na condição de repouso na posição sentada e durante as potências de 15 a 35 Watts (W) do teste de esforço físico dinâmico descontínuo do tipo degrau. Nível de significância de $\alpha=0,05$. 


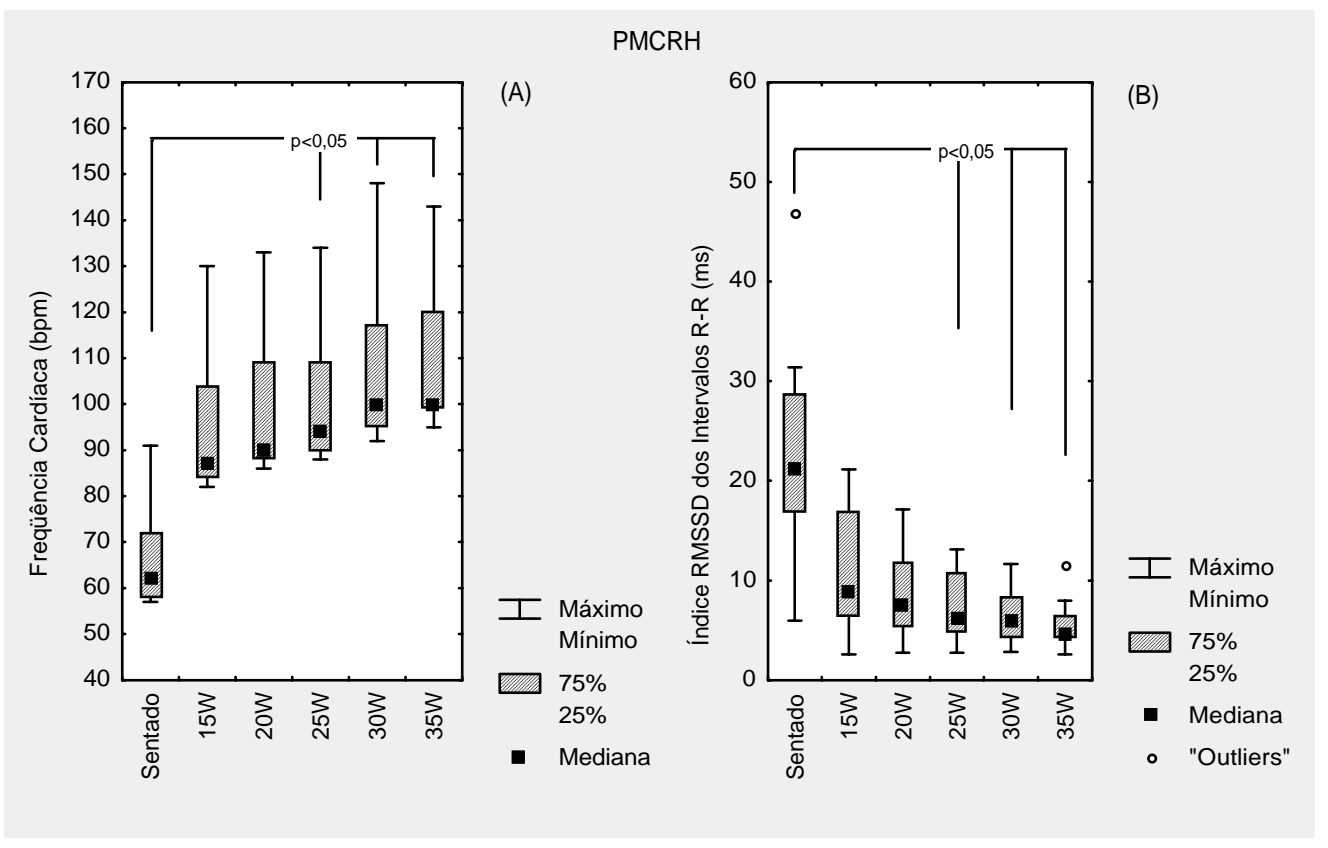

FIGURA 26. Em A, valores de freqüência cardíaca, em batimentos por minuto (bpm) e em B, dos índices de RMSSD dos intervalos R-R, em milissegundos (ms), para o grupo de voluntárias pós-menopausa com reposição hormonal $(\mathrm{PMCRH})(\mathrm{n}=9)$, na condição de repouso na posição sentada e durante as potências de 15 a 35 Watts (W) do teste de esforço físico dinâmico descontínuo do tipo degrau. Nível de significância de $\alpha=0,05$. 
Na comparação intergrupos dos valores dos índices de RMSSD dos intervalos R-R (ms) (figuras 27 a 33), verificamos que as voluntárias jovens apresentaram valores superiores deste índice em relação às voluntárias dos outros 2 grupos, na maioria dos níveis de esforço estudados. Entretanto, foi observada diferença estatisticamente significante $(p<0,05)$ apenas na potência de $35 \mathrm{~W}$ entre as jovens e as $\mathrm{PMSRH}$ e entre as jovens e as PMCRH.

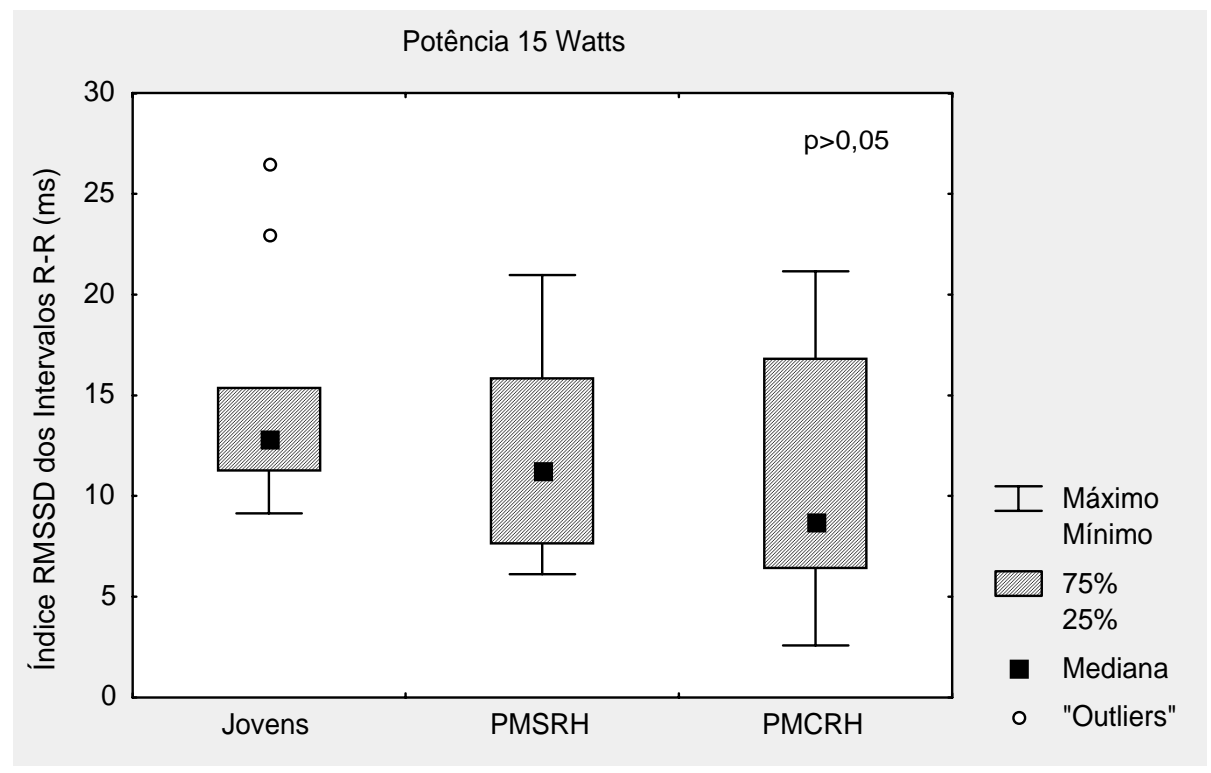

FIGURA 27. Valores dos índices de RMSSD dos intervalos R-R, em milissegundos (ms), para o grupo de voluntárias jovens $(n=10)$, pós-menopausa sem reposição hormonal (PMSRH) $(n=13)$ e pós-menopausa com reposição hormonal $(P M C R H)(n=9)$, durante a potência de 15 Watts do teste de esforço físico dinâmico descontínuo do tipo degrau. Nível de significância de $\alpha=0,05$. 


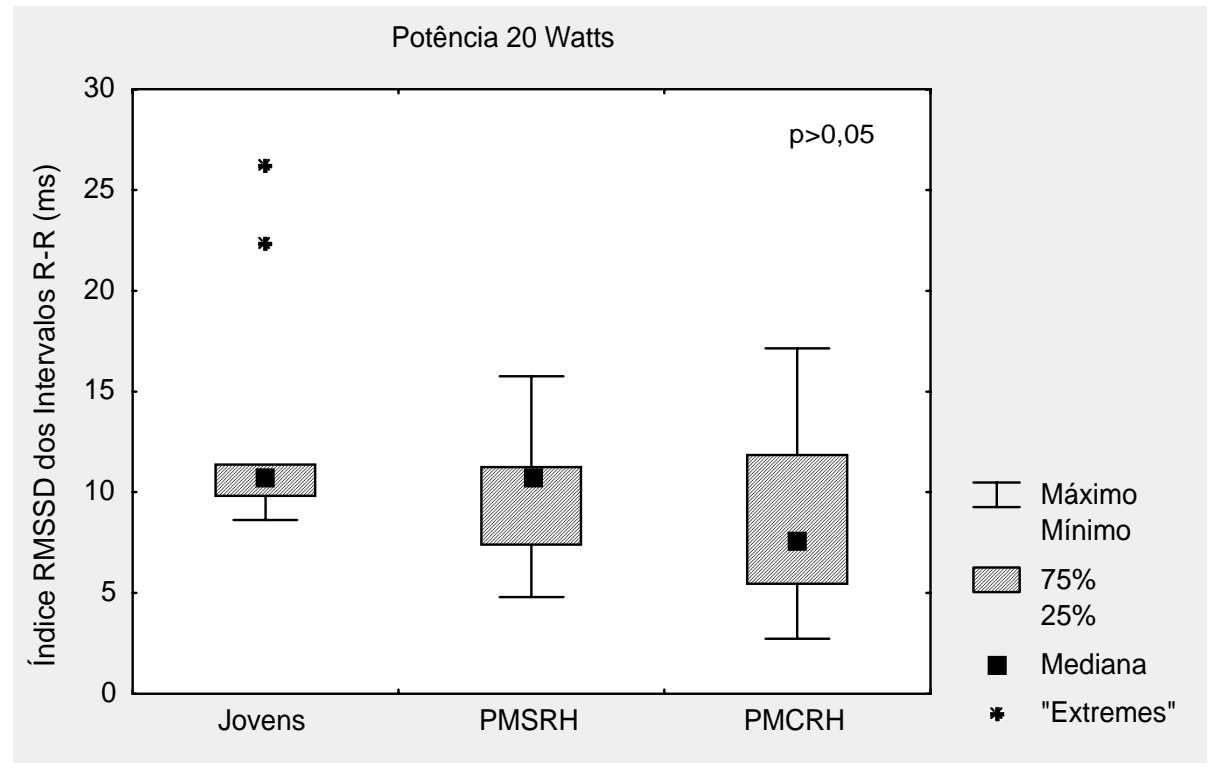

FIGURA 28. Valores dos índices de RMSSD dos intervalos R-R, em milissegundos (ms), para o grupo de voluntárias jovens $(n=10)$, pós-menopausa sem reposição hormonal (PMSRH) $(n=13)$ e pós-menopausa com reposição hormonal $(P M C R H)(n=9)$, durante a potência de 20 Watts do teste de esforço físico dinâmico descontínuo do tipo degrau. Nível de significância de $\alpha=0,05$. 


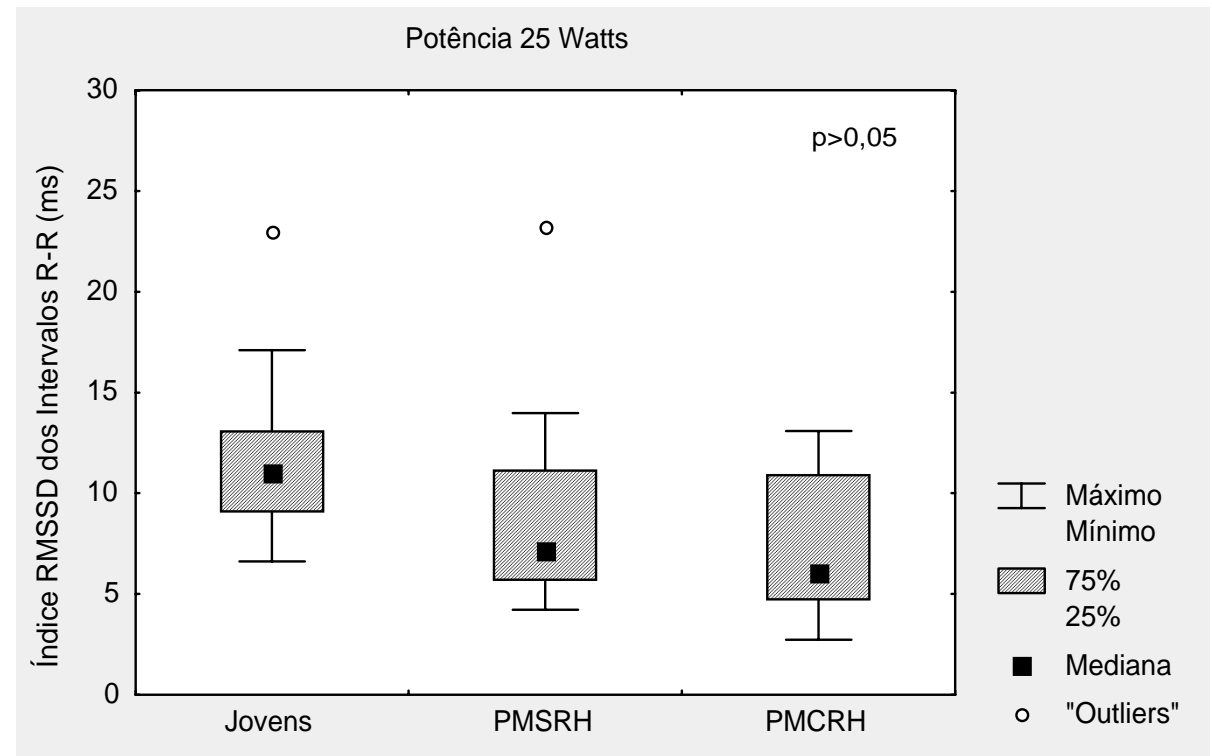

FIGURA 29. Valores dos índices de RMSSD dos intervalos R-R, em milissegundos (ms), para o grupo de voluntárias jovens $(n=10)$, pós-menopausa sem reposição hormonal (PMSRH) $(n=13)$ e pós-menopausa com reposição hormonal $(P M C R H)(n=9)$, durante a potência de 25 Watts do teste de esforço físico dinâmico descontínuo do tipo degrau. Nível de significância de $\alpha=0,05$. 


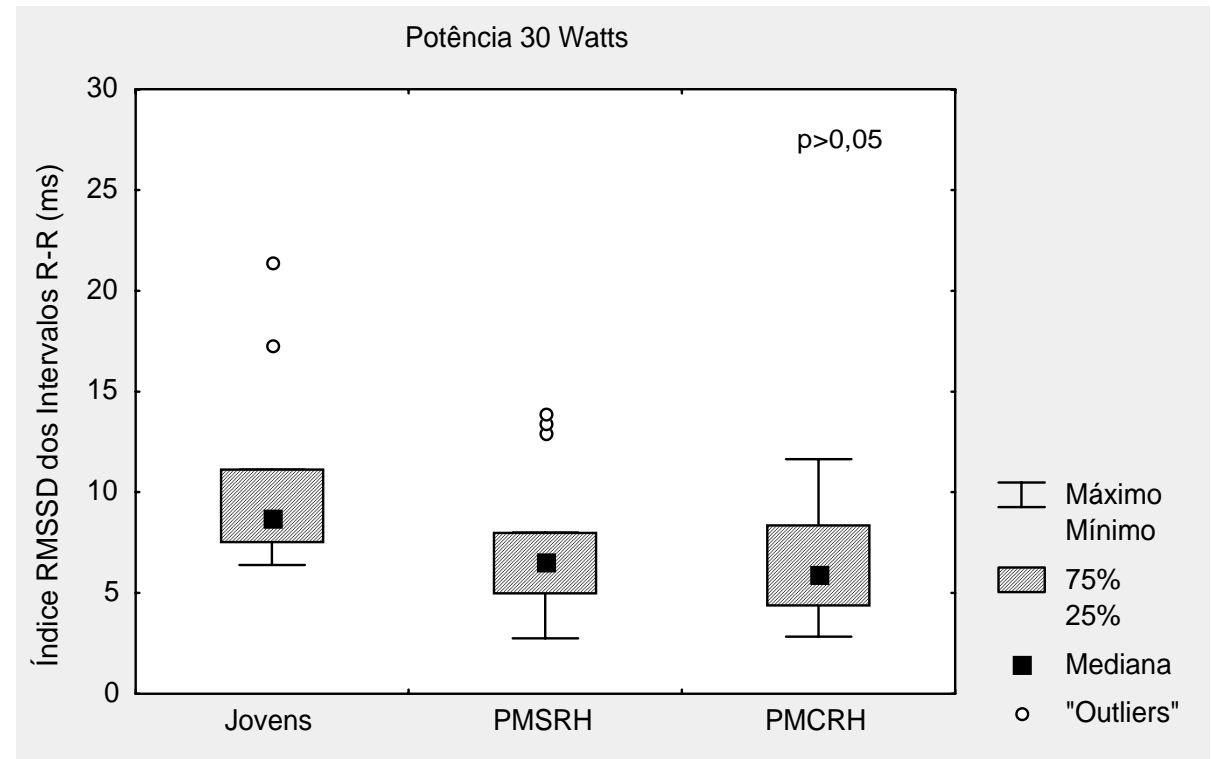

FIGURA 30. Valores dos índices de RMSSD dos intervalos R-R, em milissegundos (ms), para o grupo de voluntárias jovens $(n=10)$, pós-menopausa sem reposição hormonal (PMSRH) $(n=13)$ e pós-menopausa com reposição hormonal $(\mathrm{PMCRH})(\mathrm{n}=9)$, durante a potência de 30 Watts do teste de esforço físico dinâmico descontínuo do tipo degrau. Nível de significância de $\alpha=0,05$. 


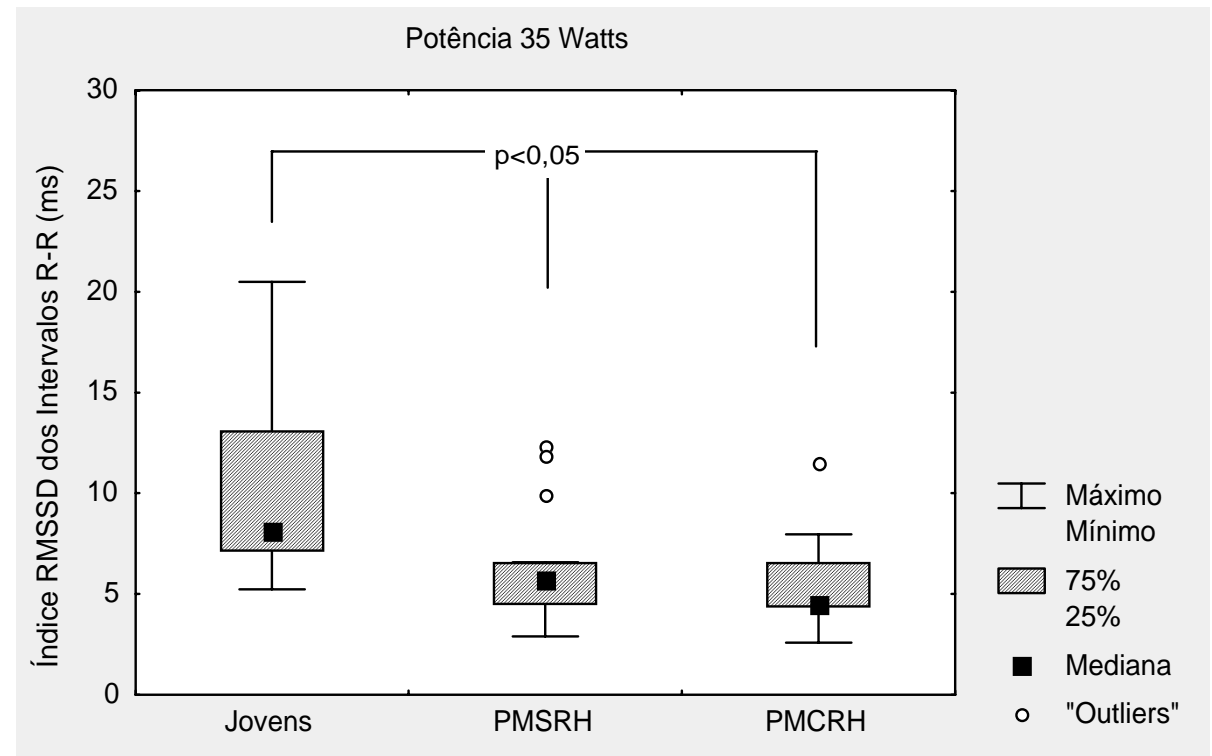

FIGURA 31. Valores dos índices de RMSSD dos intervalos R-R, em milissegundos (ms), para o grupo de voluntárias jovens $(n=10)$, pós-menopausa sem reposição hormonal (PMSRH) $(n=13)$ e pós-menopausa com reposição hormonal $(P M C R H)(n=9)$, durante a potência de 35 Watts do teste de esforço físico dinâmico descontínuo do tipo degrau. Nível de significância de $\alpha=0,05$. 


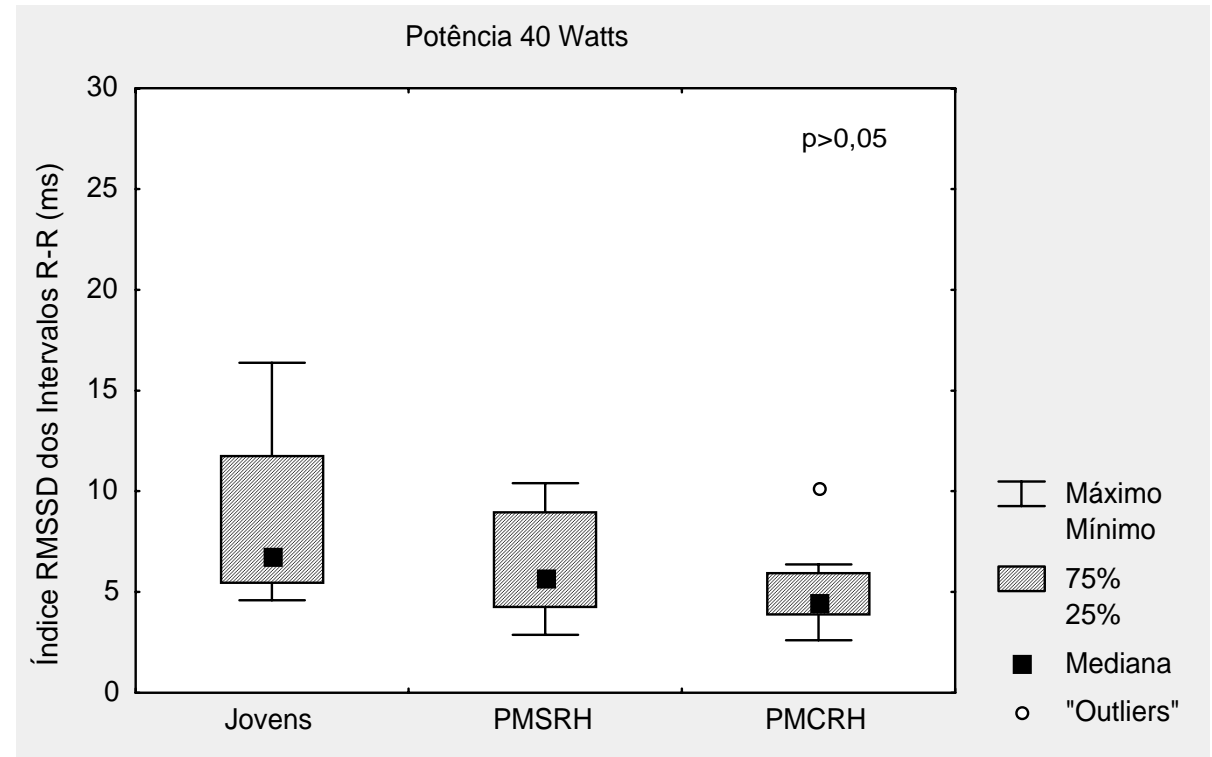

FIGURA 32. Valores dos índices de RMSSD dos intervalos R-R, em milissegundos (ms), para o grupo de voluntárias jovens $(n=10)$, pós-menopausa sem reposição hormonal (PMSRH) $(n=11)$ e pós-menopausa com reposição hormonal $(P M C R H)(n=8)$, durante a potência de 40 Watts do teste de esforço físico dinâmico descontínuo do tipo degrau. Nível de significância de $\alpha=0,05$. 


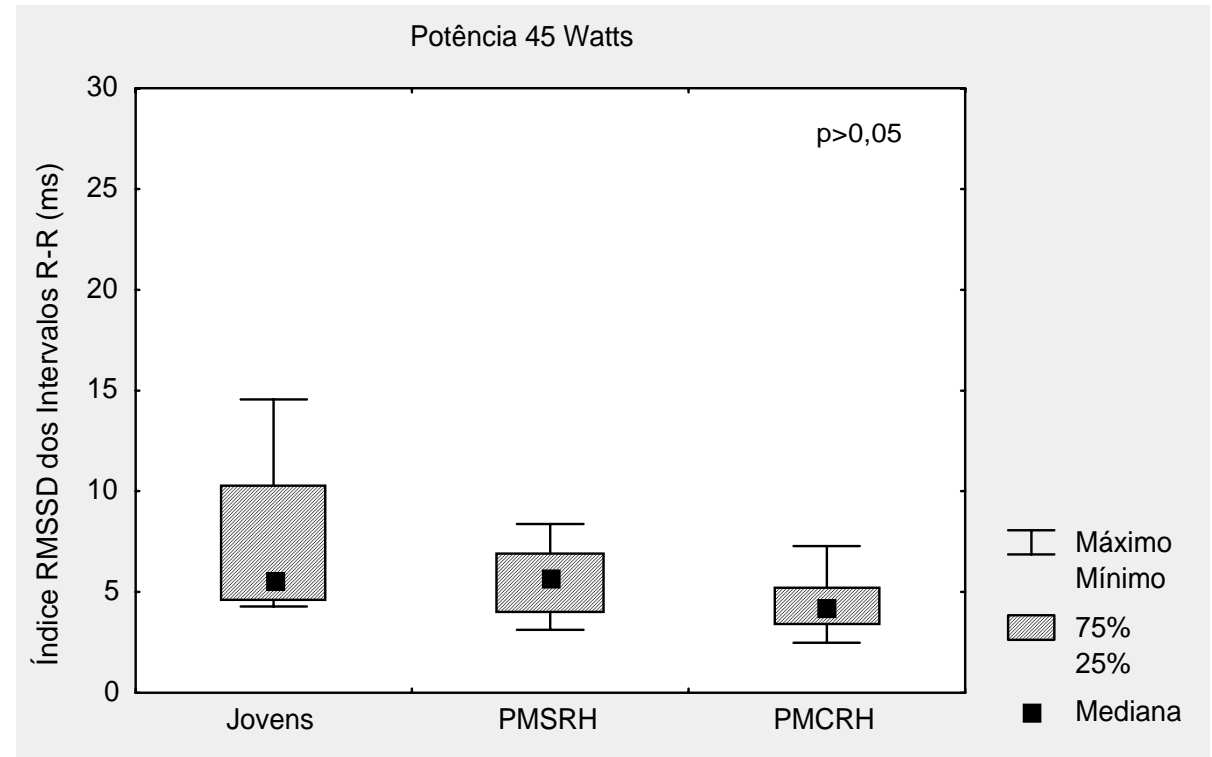

FIGURA 33. Valores dos índices de RMSSD dos intervalos R-R, em milissegundos (ms), para o grupo de voluntárias jovens $(n=10)$, pós-menopausa sem reposição hormonal (PMSRH) $(\mathrm{n}=8)$ e pós-menopausa com reposição hormonal $(P M C R H)(n=7)$, durante a potência de 45 Watts do teste de esforço físico dinâmico descontínuo do tipo degrau. Nível de significância de $\alpha=0,05$. 


\subsection{Análise da determinação do limiar de anaerobiose pelo modelo matemático e estatístico semiparamétrico, e pelo índice de RMSSD dos intervalos R-R}

Na figura 34 estão apresentados em A, os valores de potência, em W, do TEFDD-D em que foi caracterizado o LA pelo modelo SPM, e em B, os valores dos índices de RMSSD dos intervalos R-R, em ms, obtidos nos níveis de potências em que foi determinado o LA.

Em A, podemos observar que as voluntárias jovens apresentaram LA em um nível de potência superior em relação às voluntárias $\mathrm{PMSRH}$ e PMCRH, sendo esta diferença estatisticamente significante $(p<0,05)$. Em B, verifica-se que os índices de RMSSD dos intervalos $R-R$, em $m s$, foram similares entre os três grupos estudados, não apresentando diferenças estatisticamente significantes $(p>0,05)$. 


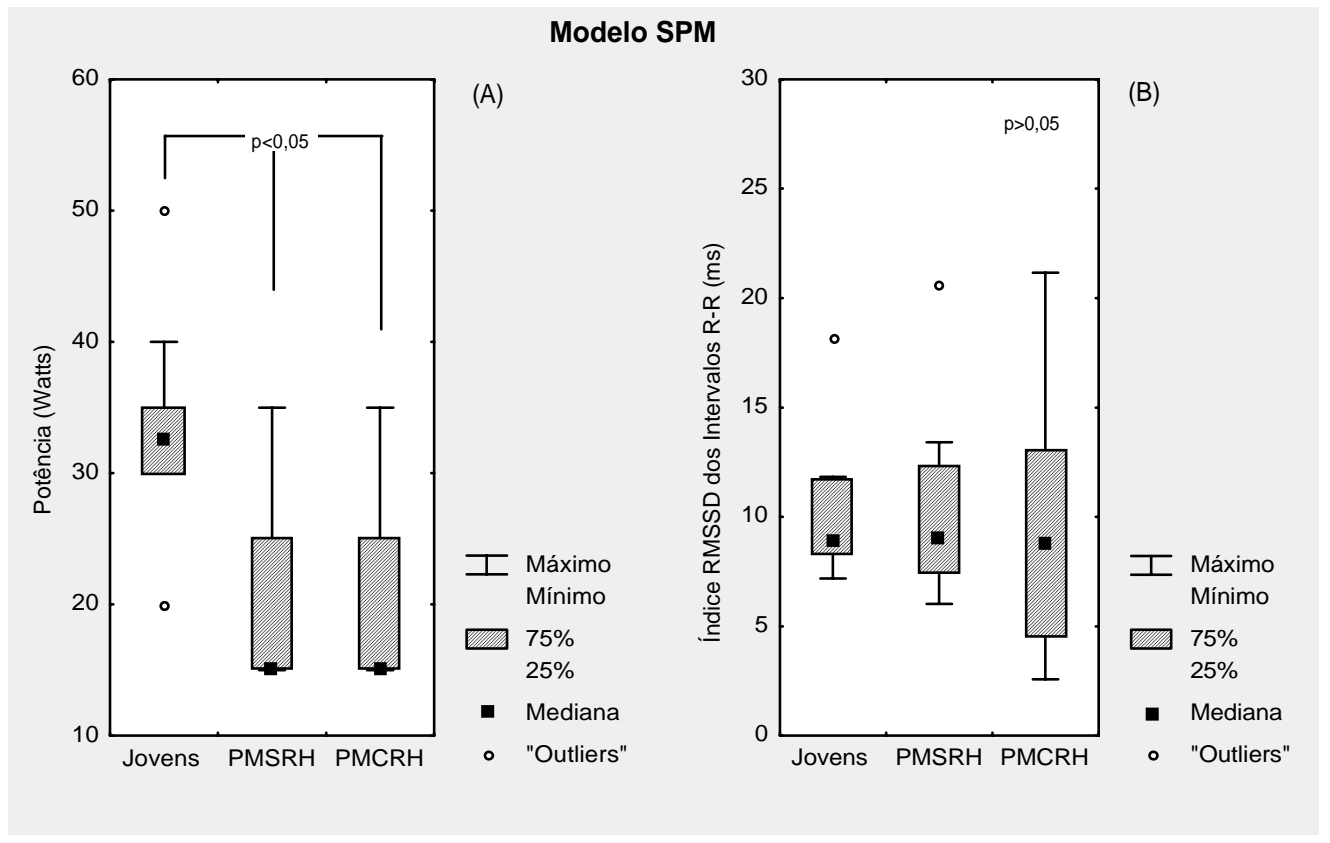

FIGURA 34. Em A, valores de potência em Watts do teste de esforço físico dinâmico descontínuo do tipo degrau e em B, dos índices de RMSSD dos intervalos R-R, em milissegundos (ms), no nível de esforço em que foi caracterizado o limiar de anaerobiose pelo modelo matemático e estatístico semiparamétrico (SPM), para o grupo de voluntárias jovens $(n=10)$, pós-menopausa sem reposição hormonal (PMSRH) $(n=13)$ e pós-menopausa com reposição hormonal $(P M C R H)(n=9)$. Nível de significância de $\alpha=0,05$. 
Pela análise estatística dos índices de RMSSD na transição do repouso na posição sentada para o exercício físico (figuras 24 B, 25 B e 26 $\mathrm{B}$, ilustradas anteriormente), o LA foi determinado na potência de $30 \mathrm{~W}$ para as jovens e de $25 \mathrm{~W}$ para as PMSRH e PMCRH.

Nas figuras 35, 36 e 37, temos a comparação dos valores do índice de RMSSD dos intervalos R-R nas potências do TEFDD-D, em que foi caracterizado o LA tanto pelo modelo SPM, como pela análise estatística dos índices de RMSSD. Podemos observar nestas figuras que os três grupos estudados não apresentaram diferenças estatisticamente significantes $(p>0,05)$ dos índices de RMSSD na comparação entre os métodos de análise. 


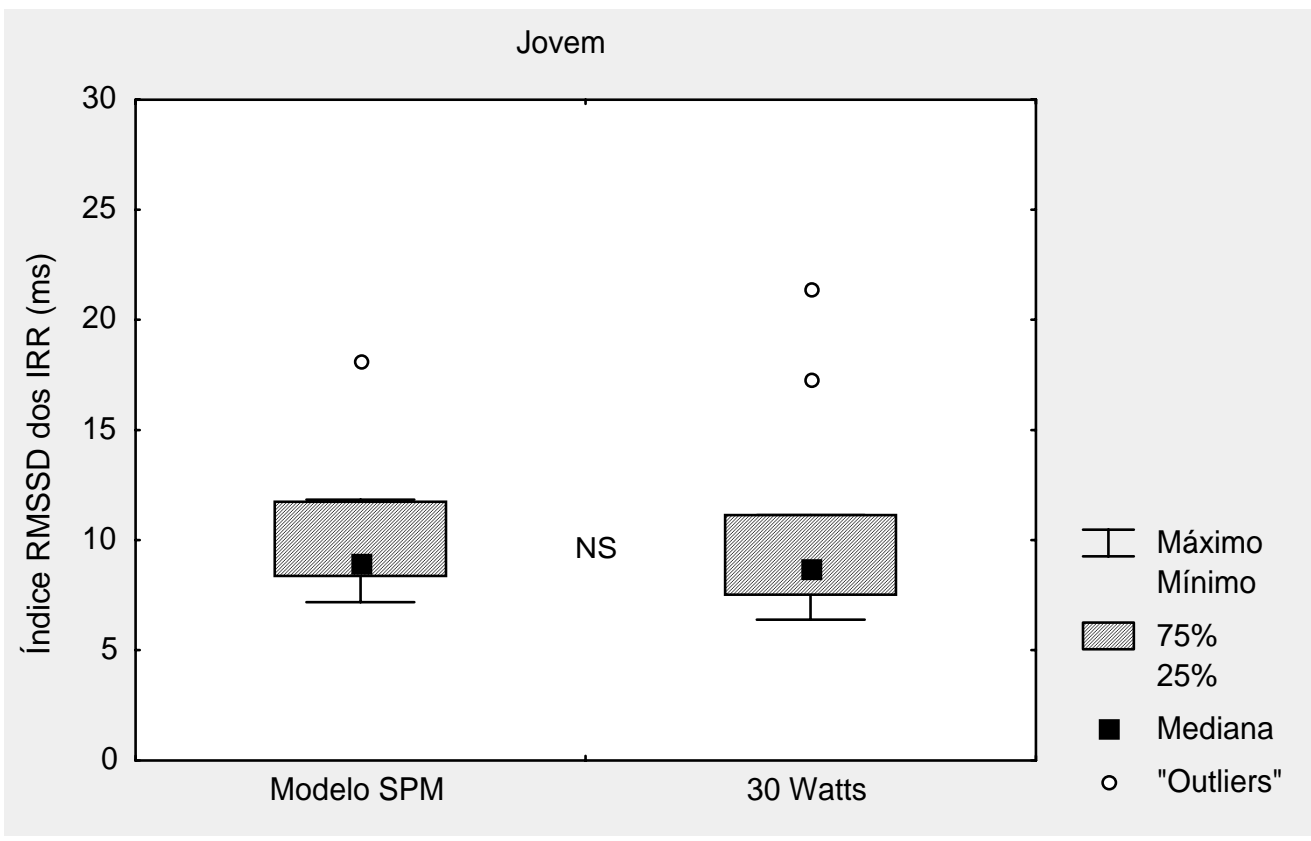

FIGURA 35. Valores do índice RMSSD dos intervalos R-R (IRR), em milissegundos (ms), nas potências do teste de esforço físico dinâmico descontínuo do tipo degrau em que foi determinado o limiar de anaerobiose pelo modelo matemático e estatístico semiparamétrico (SPM) e nas potências em que ocorreu significância estatística dos índices de RMSSD, para o grupo de voluntárias jovens $(n=10)$. Nível de significância de $\alpha=0,05$. 


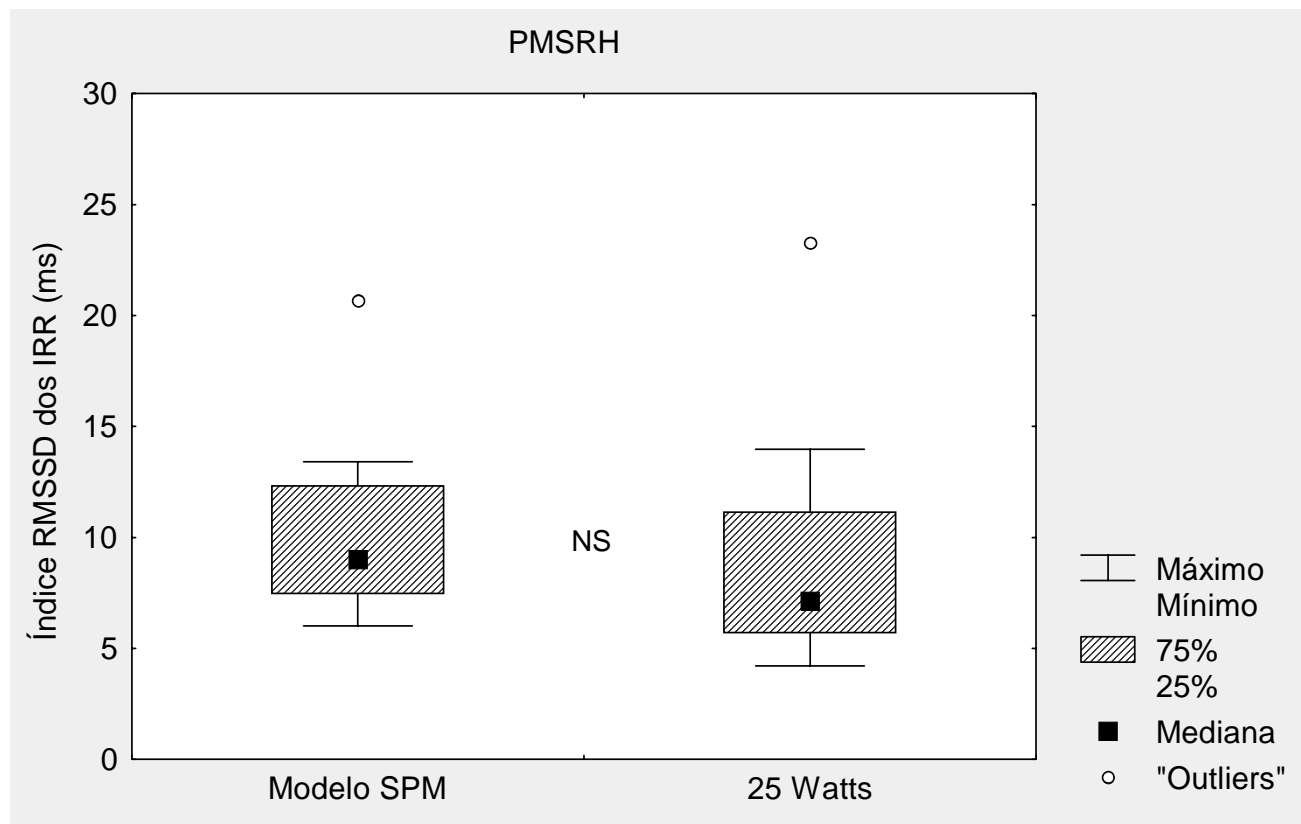

FIGURA 36. Valores do índice RMSSD dos intervalos R-R (IRR), em milissegundos (ms), nas potências do teste de esforço físico dinâmico descontínuo do tipo degrau em que foi determinado o limiar de anaerobiose pelo modelo matemático e estatístico semiparamétrico (SPM) e nas potências em que ocorreu significância estatística dos índices de RMSSD, para o grupo de voluntárias pós-menopausa sem reposição hormonal (PMSRH) ( $n=13)$. Nível de significância de $\alpha=0,05$. 
PMCRH

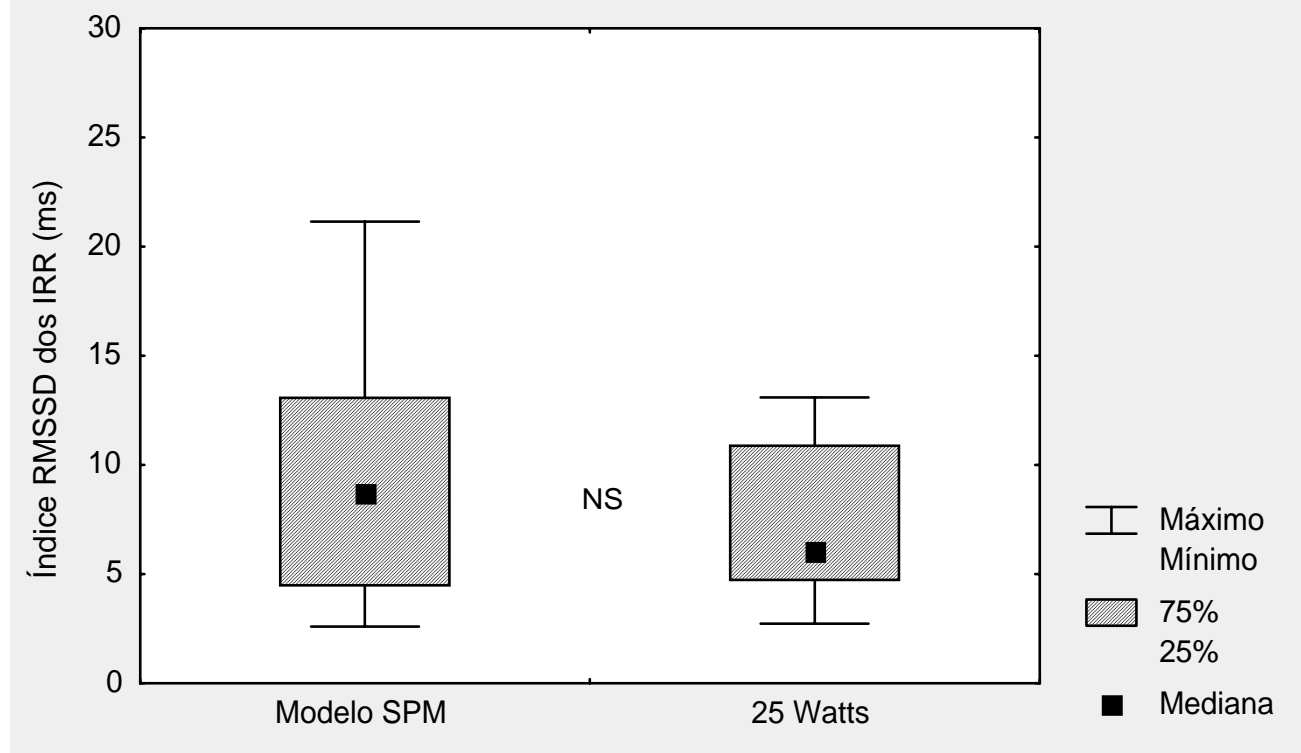

FIGURA 37. Valores do índice RMSSD dos intervalos R-R (IRR), em milissegundos (ms), nas potências do teste de esforço físico dinâmico descontínuo do tipo degrau em que foi determinado o limiar de anaerobiose pelo modelo matemático e estatístico semiparamétrico (SPM) e nas potências em que ocorreu significância estatística dos índices de RMSSD, para o grupo de voluntárias pós-menopausa com reposição hormonal (PMCRH) ( $n=9)$. Nível de significância de $\alpha=0,05$. 


\subsection{Análises de correlação}

4.7.1. Análise de correlação entre os índices de RMSSD dos intervalos R-R e as potências do teste de esforço físico dinâmico descontínuo do tipo degrau

Nas figuras de 38 a 41 estão apresentados as associações entre os valores dos índices de RMSSD dos intervalos R-R, em ms, e as potências do TEFDD-D realizadas pelas voluntárias. Verifica-se nestas figuras uma correlação estatisticamente significante $(p<0,05)$ entre as variáveis para os três grupos estudados, sendo que para as jovens o coeficiente de correlação de Spearman $\left(r_{s}\right)$ foi de $-0,49$ (figura 38); para as $P M S R H, r_{s}=-0,49$ (figura 39); para as $\mathrm{PMCRH}, \mathrm{r}_{\mathrm{s}}=-0,47$ (figura 40) e para os três grupos analisados em conjunto, $r_{\mathrm{s}}=-0,45$ (figura 41$)$. 


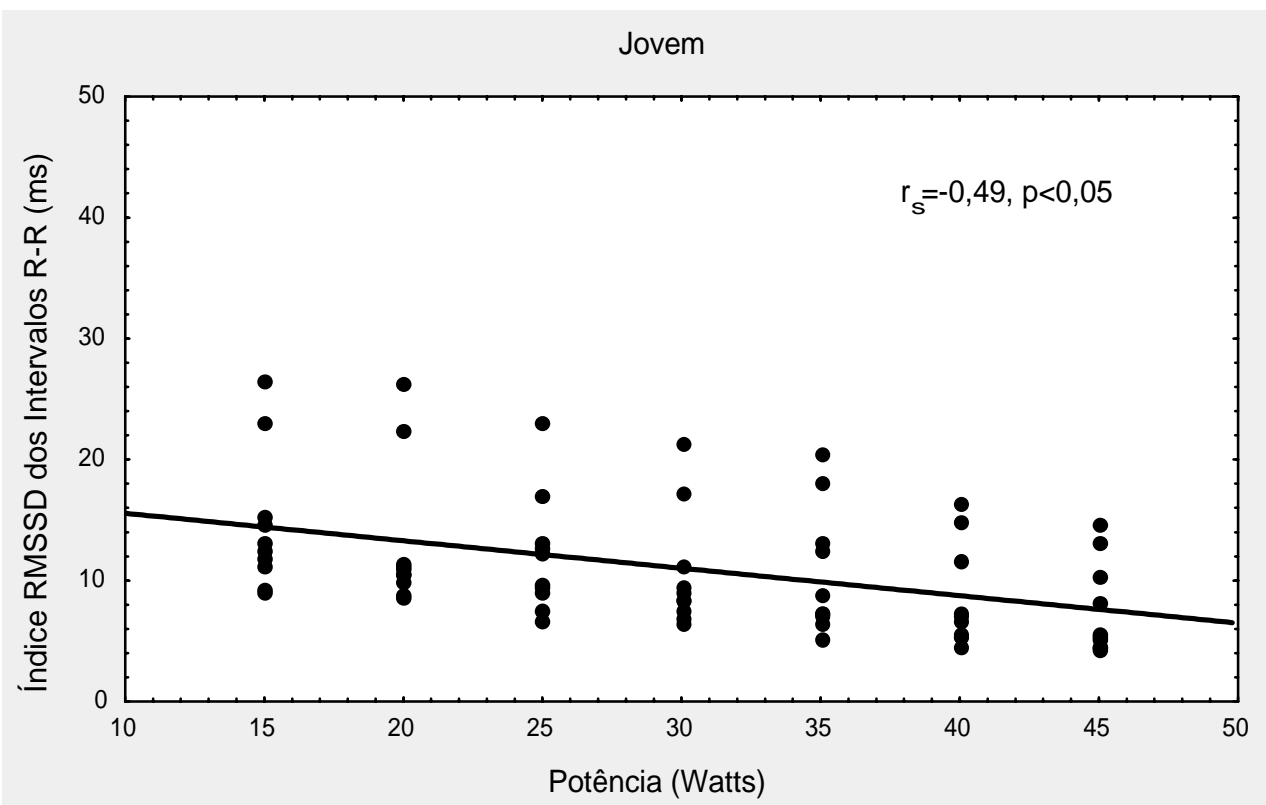

FIGURA 38. Associação dos índices de RMSSD dos intervalos R-R, em milissegundos (ms), com as potências do teste de esforço físico dinâmico descontínuo do tipo degrau realizado pelas voluntárias jovens ( $n=10)$. Nível de significância de $\alpha=0,05$. 


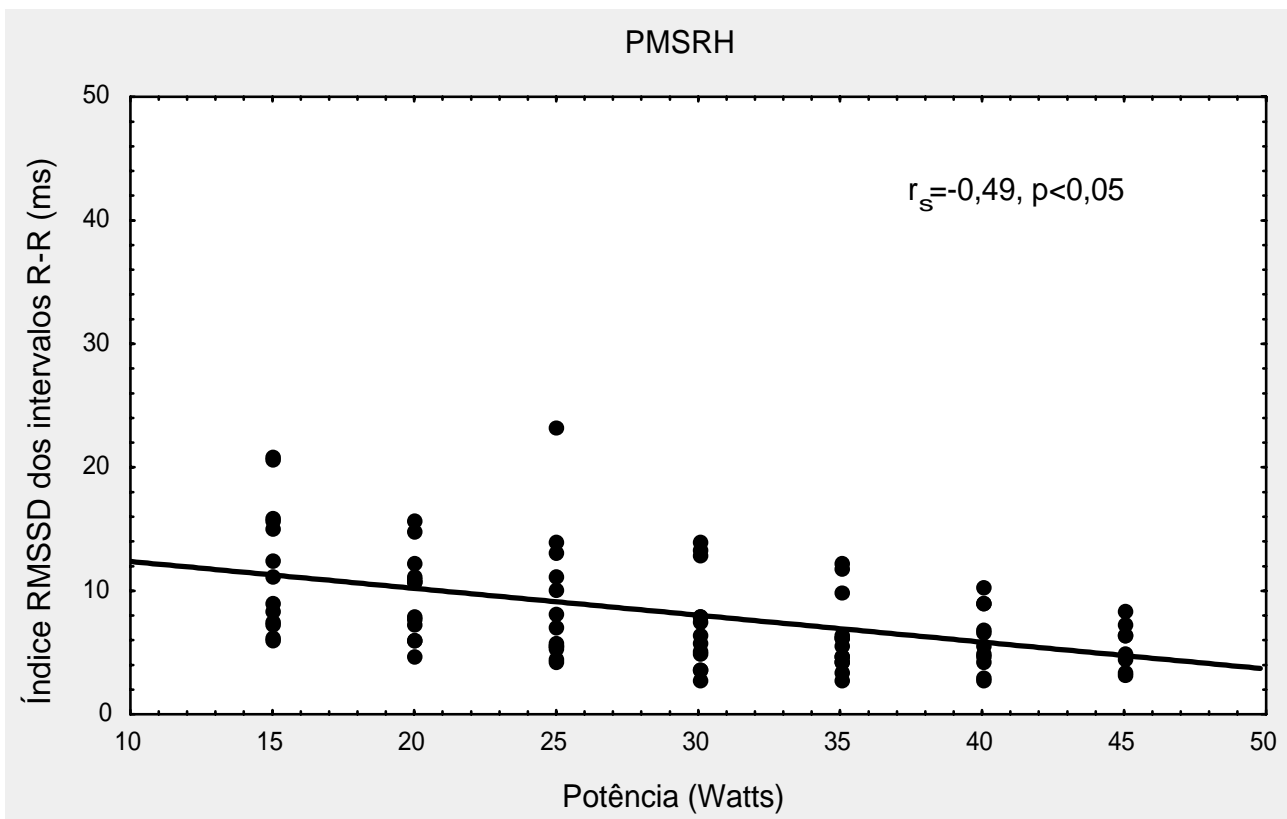

FIGURA 39. Associação dos índices de RMSSD dos intervalos $R-R$, em milissegundos (ms), com as potências do teste de esforço físico dinâmico descontínuo do tipo degrau realizado pelas voluntárias pósmenopausa sem reposição hormonal (PMSRH), sendo que nas potências de 15 a 35 Watts: $n=13$, de 40 Watts: $n=11$ e em 45 Watts: $\mathrm{n}=8$. Nível de significância de $\alpha=0,05$. 


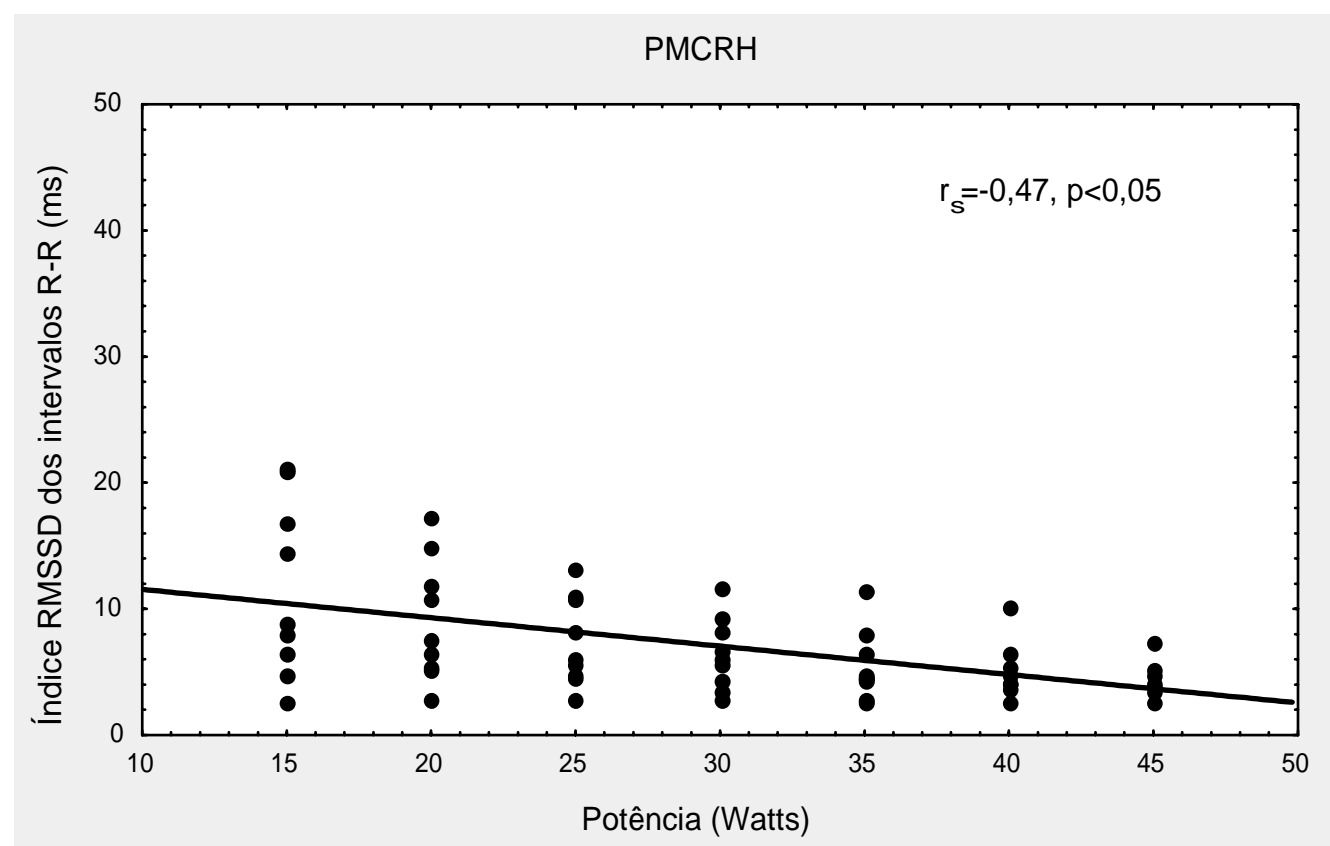

FIGURA 40. Associação dos índices de RMSSD dos intervalos R-R, em milissegundos (ms), com as potências do teste de esforço físico dinâmico descontínuo do tipo degrau realizado pelas voluntárias pósmenopausa com reposição hormonal (PMCRH), sendo que nas potências de 15 a 35 Watts: $n=9$, de 40 Watts: $n=8$ e em 45 Watts: $n=7$. Nível de significância de $\alpha=0,05$. 


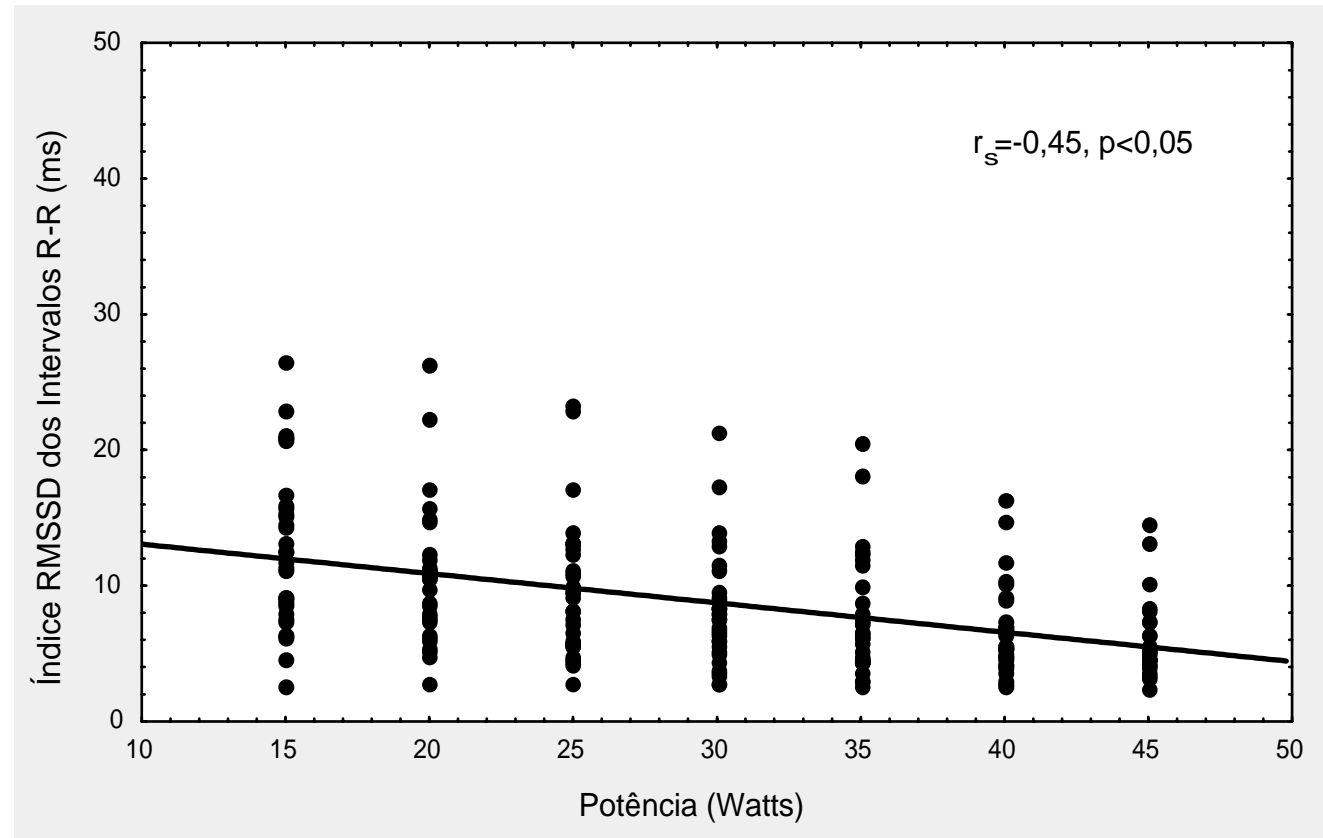

FIGURA 41. Associação dos índices de RMSSD dos intervalos R-R, em milissegundos (ms), com as potências do teste de esforço físico dinâmico descontínuo do tipo degrau realizado pelas voluntárias jovens $(n=10)$, pós-menopausa sem reposição hormonal $(P M S R H)$ $(n=13)$ e pós-menopausa com reposição hormonal (PMCRH) $(n=9)$, sendo que na potência de 40 Watts, $P M S R H: n=11$ e PMCRH: $n=8$; e em 45 Watts: PMSRH: $n=8$ e PMCRH: $n=7$. Nível de significância de $\alpha=0,05$. 
4.7.2. Análise de correlação entre os índices de RMSSD dos intervalos R-R nos níveis de potência do limiar de anaerobiose determinado pelo modelo matemático e estatístico semiparamétrico e pela análise estatística dos índices de RMSSD

Na figura 42 está apresentado a associação entre os valores dos índices de RMSSD dos intervalos R-R, em ms, nos níveis de potência em que foi determinado o LA, pelo modelo SPM e pela análise estatística dos índices de RMSSD, para os três grupos analisados em conjunto. Podemos observar nesta figura uma correlação estatisticamente significante $\left(r_{s}=0,81\right.$ e $\mathrm{p}<0,05)$ entre os índices obtidos para as duas metodologias de determinação do LA. 


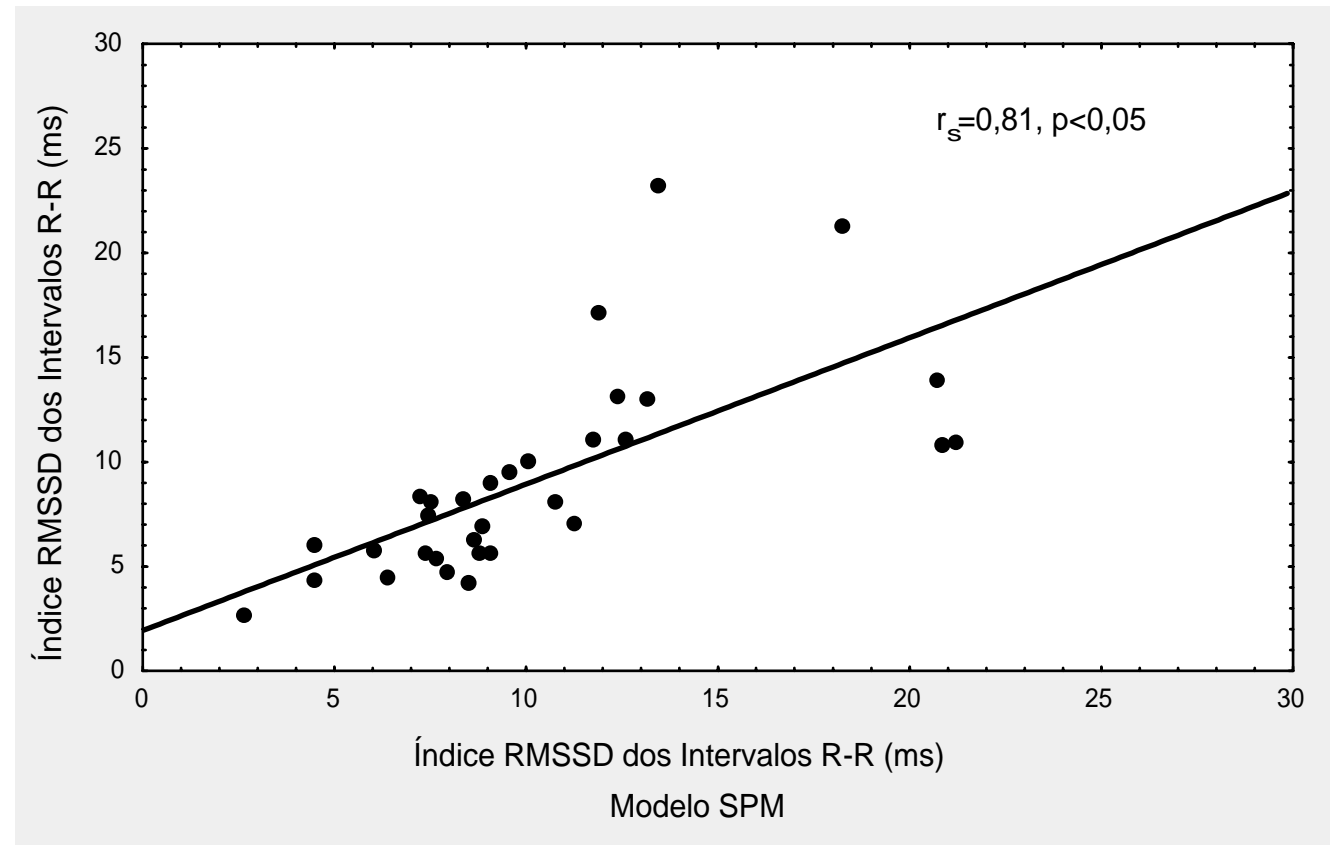

FIGURA 42. Associação entre os valores dos índices de RMSSD dos intervalos R$\mathrm{R}$, em milissegundos (ms), nos níveis de potência do teste de esforço físico dinâmico descontínuo do tipo degrau, em que ocorreu o limiar de anaerobiose, pelo modelo matemático e estatístico semiparamétrico (SPM) no eixo $\mathrm{X}$ e em $\mathrm{Y}$, no nível de potência em que ocorreu significância estatística dos índices de RMSSD, para as voluntárias jovens $(n=10)$, pós-menopausa sem reposição hormonal (PMSRH) $(n=13)$ e pós-menopausa com reposição hormonal (PMCRH) $(n=9)$, sendo que na potência de 40 Watts, PMSRH: $n=11$ e PMCRH: $n=8$; e em 45 Watts: PMSRH: $n=8$ e PMCRH: $n=7$. Nível de significância de $\alpha$ $=0,05$. 


\section{DISCUSSÃO}

\subsection{Características das voluntárias}

Diversos estudos têm demonstrado que as respostas fisiológicas dos sistemas orgânicos, tanto em repouso como durante o exercício físico são influenciadas por diversos fatores, tais como: idade, sexo, características antropométricas, hábitos de vida, nível de condicionamento físico, condição de saúde, dentre outros (GALLO Jr et al., 1995; BARBOSA et al., 1996; DAVY et al., 1998; SCHUIT et al., 1999; RIBEIRO et al., 2000; CATAl et al., 2002) Dessa forma, buscou-se neste estudo uma padronização das características individuais das participantes, para que houvesse homogeneidade entre os grupos.

Pela análise da tabela 1, podemos observar que as voluntárias jovens apresentaram valores inferiores aos apresentados pelos outros 2 grupos apenas em relação à idade, ao índice de massa corporal e a pressão arterial sistólica e diastólica. Estes dados são corroborados com dados da literatura que mostram que após a menopausa, ocorrem alterações no metabolismo lipídico e na parede vascular, contribuindo para um aumento do peso corporal e da pressão sanguínea (SANADA et al., 2000; GENAZZANI, 2001). Embora estudos tenham demonstrado que o uso de terapia de reposição hormonal contribua para reverter estas alterações (WILD, 1996; ESPELAND, 
1998; MENDELSOHN, 2000), não encontramos diferenças significativas em relação ao índice de massa corporal entre as voluntárias PMSRH e PMCRH. Verificamos ainda, que as voluntárias PMSRH e PMCRH apresentaram tempos similares de início da menopausa e o tempo de reposição hormonal das últimas foi coincidente a este período.

Em relação aos níveis hormonais (tabela 2) verificamos que os grupos na fase após a menopausa apresentam valores dentro da normalidade, ou seja, concentração sérica de FSH entre 26 e $116 \mathrm{mUl} / \mathrm{ml}$ para os 2 grupos e de estradiol menor que $30 \mathrm{pg} / \mathrm{ml}$ para as voluntárias PMSRH e menor de 93 $\mathrm{pg} / \mathrm{ml}$ para as voluntárias PMCRH.

\subsection{Análise da resposta da freqüência cardíaca e de sua variabilidade durante o repouso nas posições supina e sentada}

A modulação autonômica é o principal mecanismo responsável pelo controle da FC em indivíduos saudáveis. Durante o repouso, tanto as eferências parassimpáticas como as simpáticas estão tonicamente ativas, com efeito predominantemente vagal. A estimulação das fibras eferentes parassimpáticas está associada à diminuição dos valores de FC, enquanto que a estimulação das fibras eferentes simpáticas está associada ao aumento destes (SATO et al., 1980; MACIEL et al., 1985; MACIEL et al., 1986).

Em repouso, dois fatores parecem influenciar a FC, o nível de condicionamento físico dos indivíduos (SEALS \& CHASE, 1989; DAVY et al., 1996; TULPPO et al., 1998; LOIMAALA et al., 2000) e a posição corporal 
(BEVEGARD \& SHEPERD, 1967; SHANNON et al., 1987; DAVY et al., 1996). O envelhecimento, por sua vez, não promove qualquer modificação significativa na FC em repouso (SHANNON et al., 1987; TATE et al., 1994; TULPPO et al., 1998).

Neste estudo, as voluntárias do grupo PMCRH apresentaram menores valores absolutos de $\mathrm{FC}$ em relação aos demais grupos, tanto na posição supina como na sentada, mas sem diferenças significativas. Estes resultados são concordantes aos achados de DAVY et al. (1998), que observaram menores valores de FC em repouso em mulheres na fase da pós-menopausa, comparada as jovens. Por outro lado, CHRIST et al. (1999) observaram maiores valores de FC em repouso no grupo de mulheres em uso de terapia de reposição hormonal combinada (estrógeno associado a progesterona) em relação às mulheres que não utilizavam hormônios. No entanto, outros estudos têm demonstrado que nem a idade (SHANNON et al., 1987; TULPPO et al., 1998; MIGLIARO et al., 2001; RIBEIRO, 2001; RIBEIRO et al., 2001a) e nem a terapia de reposição hormonal (NISKANEN et al., 2002) influencia a FC de repouso.

Em relação à posição corporal, verificamos em nosso estudo maiores valores absolutos de FC na posição sentada em relação à posição supina, para os 3 grupos estudados, mas com diferença significativa apenas para as voluntárias na fase após a menopausa, com e sem reposição hormonal.

Resultados similares foram observados na literatura, que verificaram um aumento dos valores de FC na transição da posição supina para a posição sentada (MARÃES, 1999; PIKKUJÂMSÂ, et al., 2001; RIBEIRO, 
2001; RIBEIRO et al., 2001a) ou para a posição ereta (SHANNON et al., 1987; DAVY et al., 1996). Este aumento da FC ocorre em decorrência da influência da posição corporal sobre a dinâmica circulatória. Na posição supina, o volume de ejeção em repouso é quase o máximo. Por outro lado, a força da gravidade na posição ereta age contrariando o fluxo do retorno sangüíneo para o coração, resultando em volume de ejeção reduzido e FC aumentada (BEVEGARD \& SHEPHERD, 1967). Entretanto, outros estudos não observaram diferenças significativas nos valores de FC com a mudança da posição corporal, de supina para a sentada (DAVY et al., 1996).

A VFC tem sido estudada em repouso como meio não invasivo para avaliação da regulação autonômica sobre o coração. Estudos têm demonstrado importantes alterações da VFC em função da idade (SHANNON et al., 1987; BARBOSA et al., 1996; YAMASAKI et al., 1996; MIGLIARO et al., 2001; RIBEIRO, 2001; RIBEIRO et al., 2001a) e do nível de condicionamento físico (KATONA et al., 1982; GOLDSMITH et al., 1992; De MEERSMAN, 1993; DAVY et al., 1996; 1998; SCHUIT et al., 1999) em ambos os sexos. Segundo TSUJl et al. (1996), a idade é um dos determinantes mais importantes da VFC.

As reduções na VFC que ocorrem nas mulheres com o envelhecimento também podem estar relacionadas às reduções dos níveis estrogênicos que ocorrem com o advento da menopausa (MERCURO et al., 2000; RIBEIRO, 2001; RIBEIRO et al., 2001a). Este desbalanço do controle autonômico parece estar relacionado a um aumento da atividade do sistema nervoso simpático (ROSANO et al., 1997). 
Verificamos em nosso estudo, maiores valores de VFC em repouso para o grupo de voluntárias jovens, nas 2 posições estudadas, com diferença significativa em relação às voluntárias $\mathrm{PMCRH}$ na posição supina e em relação as PMSRH, na posição sentada. DAVY et al. (1998), RIBEIRO (2001) e RIBEIRO et al. (2001a) também verificaram uma diminuição significativa dos índices de VFC em mulheres na fase após a menopausa, comparada ao grupo jovem. Esta redução da VFC em função do envelhecimento também foi observada por outros autores (SHANNON et al., 1987; BARBOSA et al., 1996; MIGLIARO et al., 2001).

$\mathrm{Na}$ literatura tem sido referido que a terapia de reposição hormonal atenua as modificações que ocorrem na modulação autonômica do coração de mulheres após a menopausa. Vários autores observaram aumentos significativos dos índices de VFC com o uso de reposição hormonal com estrógeno (HUIKURI et al., 1996; ROSANO et al., 1997; MERCURO et al., 2000). Em estudo recente, analisando mulheres na fase após a menopausa que utilizavam estrógenos transdérmicos, foi observado um aumento do tônus vagal tanto em estado de vigília como durante o sono (VIRTANEN et al., 2000). Já no estudo de FARAG et al. (2002), os resultados mostraram que o uso combinado de estrógeno e progesterona foi mais favorável à modulação autonômica do coração do que o uso do estrógeno sozinho.

Entretanto, em nosso estudo não verificamos diferenças significativas dos índices de VFC entre as voluntárias na fase após a menopausa, sem e com reposição hormonal (estrógeno associado a progesterona). Resultados similares aos nossos foram observados por outros autores (DAVY et al., 
1998; NISKANEN et al., 2002). CHRIST et al. (1999) verificaram uma diminuição da VFC num grupo de mulheres na fase após a menopausa utilizando terapia de reposição hormonal em comparação a um grupo de mulheres não usuárias de hormônios. Entretanto, numa análise mais detalhada verificou-se que a redução da VFC foi restrita ao subgrupo de mulheres que utilizavam terapia de reposição hormonal combinada de estrógeno e progesterona, enquanto que as mulheres que utilizavam apenas estrógeno não apresentaram alterações na VFC.

Como podemos verificar existem muitas controvérsias a respeito dos efeitos benéficos da terapia de reposição hormonal sobre a VFC. Embora diversos estudos tenham demonstrado um importante papel do estrógeno sobre a regulação autonômica do coração, a adição de progesterona no tratamento parece neutralizar este efeito. Entretanto na prática clínica recomenda-se a utilização de estrógeno combinado a progesterona devido a ação protetora deste último sobre o endométrio, evitando o aparecimento de lesões hiperplásicas (WHITEHEAD et al., 1990; GRADY et al., 1992).

Quanto à posição corporal, não observamos diferenças significativas nos valores de VFC entre as posições supina e sentada, tanto para as voluntárias jovens, como para as $\mathrm{PMCRH}$. Outros estudos também não observaram alterações nos índices temporais de VFC com a mudança postural (MARÃES, 1999; RIBEIRO, 2001; RIBEIRO et al., 2001a; SILVA et al., 2001). Já as voluntárias PMSRH apresentaram valores de VFC significativamente superiores durante o repouso na posição supina. DAVY et al. (1996) estudando mulheres na fase após a menopausa, observaram uma 
diminuição significativa dos índices de VFC da posição supina para a ereta. O mesmo foi observado por LINDQVIST (1990).

Embora tem sido referido na literatura que ocorrem variações no tônus vago-simpático sobre o sistema cardiovascular durante a mudança postural, ou seja, aumento da atividade simpática e retirada vagal com a transição da posição supina para ereta (SHANNON et al., 1987), em nosso estudo, verificamos que estas variações nem sempre são refletidas pelos índices temporais de VFC.

\subsection{Análise da resposta da freqüência cardíaca e de sua variabilidade ao exercício físico dinâmico}

Diversos estudos têm demonstrado que a taquicardia induzida pelo exercício dinâmico é mediada por modificações na atividade dos eferentes simpático e parassimpático e que a relativa contribuição destas eferências estão na dependência da intensidade e duração do esforço (ROBINSON et al., 1966; NORDENFELT, 1971; MACIEL et al., 1986; GALLO Jr et al., 1988; 1995; SEALS \& VÍCTOR, 1991; NÓBREGA \& ARAÚJO, 1993).

No início do exercício, impulsos aferentes provenientes do córtex motor e dos mecanorreceptores incidem sobre a área cardiovascular no bulbo, levando a uma inibição da atividade das vias eferentes do sistema nervoso parassimpático sobre o coração e acarretando um aumento rápido da FC (MITCHELL, 1990; NÓBREGA \& ARAÚJO, 1993; WILLIANSON et al., 1995). 
Essa elevação rápida da FC, constitui um mecanismo muito importante no sentido de aumentar o fluxo sangüíneo propiciando uma maior eficiência do mecanismo de transporte de oxigênio aos músculos em atividade contráctil (GALLO Jr et al., 1990; BARROS NETO, 1996).

Verificamos em nosso estudo, pela análise visual, que o padrão de resposta da FC nos diferentes níveis de esforço estudado foi semelhante ao descrito na literatura, ou seja, no início do exercício observa-se um acentuado aumento da FC, que pode ser atribuído à diminuição da estimulação parassimpática sobre o nódulo sinusal, o qual independe da intensidade da potência aplicada. Em seguida, verifica-se um decréscimo da FC, decorrente da retomada vagal (MACIEL et al., 1986; SILVA, 1988; GALLO Jr et al., 1988; 1995; NÓBREGA \& ARAÚJO, 1993; CATAI, 1999; SILVA et al., 2001).

Quanto à magnitude da variação da FC no início do exercício físico, esta parece ser dependente da idade. Em nosso estudo, as voluntárias jovens apresentaram maiores valores de variação da $\mathrm{FC}$ em relação às voluntárias PMSRH, com significância estatística na maioria das potências de esforço estudadas. Esses resultados são concordantes com os observados por RIBEIRO (2001), que encontrou maiores valores de variação da FC para as jovens comparativamente às mulheres na fase após a menopausa que não utilizavam reposição hormonal. Já as voluntárias PMCRH apresentaram valores inferiores às jovens, porém sem significância estatística. As variações de FC das mulheres PMCRH foram superiores as PMSRH, porém não apresentando diferenças significantes, o que nos leva a 
supor que a terapia de reposição hormonal pode ter influenciado na dinâmica vagal no início do exercício. Ressalta-se que em um estudo com mulheres na fase após a menopausa com e sem reposição hormonal submetidas a treinamento físico não foi evidenciado diferenças na variação da FC (FORTI, 1999). SEALS et al. (1994) referem que com o aumento da idade ocorre uma redução da modulação vagal sobre o coração, fato este que parece limitar a capacidade da retirada vagal atenuando a taquicardia rápida em resposta a certos estímulos fisiológicos.

Vários autores (MACIEL et al., 1986; GALLO Jr et al., 1989; CHACON-MIKAHIL et al., 1998; CATAI, 1999; FORTI, 1999) têm estudado a variação da FC no início do exercício em função de intervalos de tempo préestabelecidos, ou seja, no período de 0 a $10 \mathrm{~s}$ e de 0 a $30 \mathrm{~s}$, que são predominantemente vago-dependentes. No entanto, outros autores (SILVA, 1988; RIBEIRO, 2001) demonstraram que o período de tempo para que ocorra a elevação rápida da FC não é fixo, ou seja, a retirada vagal, se estabelece de forma individual, com tempos distintos. O mesmo foi observado em nosso estudo, onde as voluntárias jovens e as PMSRH apresentaram tempo de variação da FC entre 12 e 14 s e as PMCRH entre 10 e 14 s, nas diferentes potências de esforço estudadas. Além disso, verificamos que nem a idade e nem a terapia de reposição hormonal influenciaram o tempo de retirada vagal.

Com relação ao padrão da resposta da FC, após a fase inicial do exercício, nossos dados foram similares aos observados em outros estudos (ROBINSON et al., 1966; NORDENFELT, 1971; MACIEL et al., 1986; SILVA, 
1988; GALLO Jr et al., 1988; 1989, 1995; SILVA et al., 2001), que verificaram que em baixas potências do esforço, a FC pode se estabilizar ou até reduzir durante todo o período de exercício, comportamento este mediado predominantemente pela retirada vagal. Já em níveis de potência mais elevados é evidenciado um acréscimo mais lento da FC, o qual depende quase exclusivamente da contribuição da atividade simpática sobre o nódulo sinusal e esta atividade aumenta de intensidade proporcionalmente ao aumento do nível de esforço.

Este aumento da atividade simpática sobre o coração pode ser explicada pela ação das catecolaminas circulantes (CHRISTENSEN \& GALBO, 1983; ROWELL, 1986) e por estímulos provenientes dos metabolorreceptores dos músculos em atividade (MITCHELL, 1990; MIDDLEKAUFF et al., 1997), afim de atender a demanda metabólica dos mesmos.

No presente estudo, a análise simultânea da FC e de sua variabilidade, por meio dos índices de RMSSD dos intervalos R-R, durante o exercício físico dinâmico descontínuo, demonstrou existir uma relação inversa entre as mesmas. Enquanto a FC aumentou progressivamente com o incremento de potência, a VFC diminuiu. Isto foi observado para os três grupos estudados. Resultados similares foram encontrados no estudo de ALONSO et al. (1998), que utilizaram protocolo em degrau contínuo. Verifica-se ainda neste estudo, pela análise de correlação, que existe uma associação significativa entre os índices de RMSSD dos intervalos R-R e as potências do TEFDD-D estudadas, para todos os grupos. 
Podemos observar também que o nível de esforço em que foi detectado um aumento significativo da FC em relação ao repouso foi similar ao observado pela análise da VFC, ou seja, na potência de $30 \mathrm{~W}$ para as jovens e de $25 \mathrm{~W}$ para as PMCRH. Já as PMSRH apresentaram aumento significativo da FC na potência de $20 \mathrm{~W}$ e diminuição significativa da VFC em $25 \mathrm{~W}$, no entanto esses níveis de potências são muito próximos. Estes dados sugerem que as diferenças estatisticamente significantes da FC durante a realização de exercício físico dinâmico ocorrem em níveis de potências em que há uma diminuição da atividade do sistema parassimpático.

Quando comparamos os grupos estudados em relação aos valores dos índices de RMSSD dos intervalos R-R obtidos durantes as potências dos TEFDD-D, verificamos que os mesmos são aparentemente maiores para o grupo de voluntárias jovens, porém com significância estatística apenas na potência de $35 \mathrm{~W}$, sugerindo que as modificações na modulação parassimpática durante o exercício sejam semelhantes entre os três grupos estudados. MARÃES (1999) também não observou diferenças significativas dos índices de VFC no domínio do tempo (RMSM e RMSSD) entre homens jovens e de meia-idade. 


\subsection{Identificação do limiar de anaerobiose pelo modelo matemático e estatístico semiparamétrico e pela variabilidade da freqüência cardíaca}

O LA é um importante parâmetro indicador da capacidade física dos indivíduos, que corresponde ao momento no qual o metabolismo anaeróbio passa a ter importância na produção de energia, com acúmulo simultâneo de ácido láctico nos músculos e conseqüentemente na circulação sangüínea (BARROS NETO, 1996; CHACON-MIKAHIL et al., 1998; WASSERMAN et al., 1999).

Os 2 métodos mais comumente utilizados para detecção do LA são: um invasivo, por meio da dosagem do lactato sangüíneo (COYLE et al., 1983; WELTMAN et al., 1990; FOSTER et al., 1999) e o outro não-invasivo, por meio da mudança do padrão das respostas das curvas ventilatórias durante o exercício físico dinâmico contínuo e incremental (WASSERMAN et al., 1990; 1999; CHACON-MIKAHIL, 1998; CHACON-MIKAHIL et al., 1998; CATAI, 1999; FORTI, 1999).

Entretanto, tem sido proposto em nosso laboratório, a utilização da análise da perda da estabilidade da resposta da FC para estimar o LA, por meio da aplicação do modelo auto-regressivo integrado médias móveis (ARIMA) (MARÃES et al., 1996; 2000b; MARÃES, 1999; RIBEIRO et al., 1998) ou do modelo matemático e estatístico semiparamétrico (SPM) (RIBEIRO et al., 2001b; FERREIRA et al., 2002; SAKABE et al., 2002).

Pelo modelo SPM, o LA foi caracterizado durante o TEFDD-D, como o momento em que a resposta da FC apresentou uma inclinação positiva e 
estatisticamente significante. MARÃES (1999) e RIBEIRO et al. (2001b) referem que a perda da estabilidade da FC se deve ao predomínio da atividade simpática sobre o nódulo sinusal. Por esta análise, as voluntárias jovens atingiram o LA em potências do TEFDD-D significativamente maiores em relação aos outros 2 grupos estudados. Estes resultados estão de acordo com dados observados na literatura que mostram uma redução progressiva na capacidade aeróbia dos indivíduos com o aumento da idade (CATAI, 1999; MARÃES, 1999; RIBEIRO et al., 2001b; DAVY et al., 1998). Quanto à terapia de reposição hormonal, verificamos que a mesma não interferiu no desempenho físico das voluntárias, uma vez que não foram observadas diferenças entre os grupos PMSRH e PMCRH. Resultados similares foram observados no estudo de FORTI (1999). Comparando os valores dos índices de RMSSD dos intervalos $\mathrm{R}-\mathrm{R}$, obtidos para cada potência do TEFDD-D em que foi caracterizado o LA pelo modelo SPM, não observamos diferenças significativas entre os grupos.

Uma outra metodologia utilizada neste estudo para determinar o LA, foi a baseada na análise dos índices de RMSSD dos intervalos $\mathrm{R}-\mathrm{R}$, devido às suas propriedades estatísticas e por existir uma forte correlação entre estes índices e a magnitude do tônus vagal (LONGO et al., 1995; TASKE FORCE, 1996), Diversos estudos na literatura têm associado o LA com o momento de transição do predomínio da atividade parassimpática para a simpática (MACIEL et al., 1986; GALLO Jr et al., 1988; 1995; YAMAMOTO et al., 1991; TULPPO et al., 1996; ALONSO et al., 1998). Assim, em nosso estudo, o nível de esforço físico em que foi observada uma redução 
significativa do índice de RMSSD dos intervalos R-R em relação ao repouso, foi considerado como o momento em que se atingiu o LA.

Por este tipo de análise, a potência do TEFDD-D em que foi caracterizado o LA também foi superior para as voluntárias jovens em relação aos outros 2 grupos, sendo que para o grupo das jovens o LA foi estimado na potência de $30 \mathrm{~W}$ e para os grupos das PMSRH e PMCRH, na potência de $25 \mathrm{~W}$. A partir destas potências, a VFC manteve-se inferior e observa-se um achatamento da mesma, porém com significância estatística em relação aos valores de repouso, até a potência máxima estudada para cada grupo. ALONSO et al. (1998) estudando o comportamento da VFC (desvio padrão dos intervalos $\mathrm{R}-\mathrm{R}$ ) durante exercício físico progressivo, também observou uma diminuição significativa da VFC do repouso até o LA e, a partir deste ponto, mantendo-se inalterada até o pico do exercício. Estes resultados são corroborados com os achado de outros autores (YAMAMOTO et al., 1991; TULPPO et al., 1996) que mostram uma associação entre a retirada vagal e o limiar de anaerobiose.

Comparando os valores dos índices de RMSSD dos intervalos R-R, nos níveis de potência em que foi estimado o LA, pelo modelo SPM e pela análise estatística dos índices de RMSSD dos intervalos $R-R$, não observamos diferenças estatisticamente significantes entre os mesmos para os três grupos estudados. A análise de correlação mostra ainda uma forte associação entre os mesmos. Estes resultados são concordantes com SAKABE et al. (2002) que referem que a mudança do padrão da resposta de FC analisado pelo modelo SPM coincide com a diminuição da VFC. 


\section{CONCLUSÃO}

A partir da análise do conjunto de resultados obtidos no presente estudo, dentro da condição experimental empregada, pode-se chegar as seguintes conclusões:

a. Os menores valores dos índices de RMSSD dos intervalos R-R observados para as voluntárias na fase após a menopausa (PMSRH e PMCRH) durante o repouso e em exercício físico dinâmico, bem como a menor magnitude de variação da FC na fase inicial do exercício, podem estar relacionados a uma redução na atividade parassimpática sobre o coração, decorrente principalmente do processo de envelhecimento e da menor capacidade física observada nestas voluntárias. Embora as alterações hormonais possam ter alguma influência nestes resultados, a terapia de reposição hormonal não contribuiu de forma a reverter os mesmos.

b. As duas metodologias de análise do LA utilizadas neste estudo se mostraram altamente correlacionáveis, o que sugere que a mudança de inclinação da resposta da FC ocorre em níveis de esforço em que os índices de RMSSD dos intervalos R-R já atingiram seus valores mínimos, em decorrência do aumento da atividade simpática sobre o nódulo sinusal. 


\section{REFERÊNCIAS BIBLIOGRÁFICAS}

AHMED, M.W.; KADISH, A.H.; PARKER, M.A.; GOLDBERGER, J.J. Effect of physiologic and pharmacologic adrenergic stimulation on heart rate variability. $\mathbf{J}$ Am Coll Cardiol, v. 24, n. 4, p. 1082-1090, 1994.

ALONSO, D.O.; FORJAZ, C.L.M.; REZENDE, L.O.; BRAGA, A.M.F.W.; BARRETO, A.C.P.; NEGRÃO, C.E.; RONDON, M.U.P.B. Comportamento da freqüência cardíaca e da sua variabilidade durante as diferentes fases do exercício físico progressivo máximo. Arq Bras Cardiol, v. 71, n. 6, p. 787-792, 1998.

AMERICAN COLLEGE OF CARDIOLOGY CARDIOVASCULAR TECHNOLOGY ASSESSMENT COMMITTEE. Heart rate variability for risk stratification of lifethreatening arrhythmias. J Am Coll Cardiol, v. 22, n. 3, p. 948-950, 1993.

ANTILA, K. Quantitative characterization of heart rate during exercise. Scand J Clin Lab Invest, v. 153, p. 3-68, 1979 (Suppl).

BARBOSA, P.R.; BARBOSA FILHO, J.; MORAIS DE SÁ, C.A. Influência da idade, sexo, e doença coronária sobre a modulação autonômica do coração. Arq Bras Cardiol, v. 67, n. 5, p. 325-329, 1996.

BARBOSA FILHO, J.; BARBOSA, P.R.B.; CORDOVIL, I. Modulação autonômica do coração na hipertensão arterial sistêmica. Arq Bras Cardiol, v. 78, n. 2, p. 181-188, 2002.

BARROS NETO, T.L. Fisiologia do exercício aplicada ao sistema cardiovascular. Rev Soc Cardiol Estado de São Paulo, v. 6, n. 1, p. 6-10, 1996

BATHIA, N.N.; BERGMAN, A.; KARRAM, M.M. Effects of estrogen on urethral function in women with urinary incontinence. Am J Obstet Gynecol, v. 160, n. 1, p. 176-181, 1989.

BEMBEN, D.A.; SALM, P.C.; SALM, A.J. Ventilatory and blood lactate responses to maximal treadmill exercise during the menstrual cycle. J Sports Med Phys Fitness, v. 35, n. 4, p. 257-262, 1995. 
BEVEGARD, B.S.; SHEPHERD, J.T. Regulation of the circulation during exercise in man. Physiol Rev, v. 47, n. 2, p. 178-213, 1967.

BIGGER, J.T.; FLEISS, J.L.; STEINMAN, R.C.; ROLNITZKY, L.M.; KLEIGER, R.E.; ROTTMAN, J.N. Frequency domain measures of heart period variability and mortality after myocardial infarction. Circulation, v. 85, n. 1, p. 164-171, 1992.

CATAI, A.M. Estudo da variabilidade da freqüência cardíaca e da capacidade aeróbia em homens jovens e de meia idade submetidos a treinamento físico aeróbio. 1999. 301p. Tese (Doutorado em Ciências) - Instituto de Biologia, Universidade Estadual de Campinas, Campinas.

CATAI, A.M.; CHACON-MIKAHIL, M.P.T.; MARTINELLI, F.S.; FORTI, V.A.M.; GOLFETTI, R.; MARTINS, L.E.B.; LIMA-FILHO, E.C.; SILVA, E.; GALLO Jr., L. Aerobic capacity and heart rate variability responses in young and middle-aged men. In: 47 ANNUAL MEETING, 2000, Indianapolis. Resumos..., Indianapolis: Medicine \& Science in Sports \& Exercise, 2000, v. 32, n. 5, p. 64 (Suppl).

CATAI, A.M.; CHACON-MIKAHIL, M.P.T.; MARTINELLI, F.S.; FORTI, V.A.M.; SILVA, E.; GOLFETTI, R.; MARTINS, L.E.B.; SZRAJER, J.S.; WANDERLEY, J.S.; LIMA-FILHO, E.C.; MILAN, L.A.; MARIN-NETO, J.A.; MACIEL, B.C.; GALLO Jr.; L. Effects of aerobic exercise training on heart rate variability during wakefulness and sleep and cardiorespiratory responses of young and middle-aged healthy men. Braz J Med Biol Res, v. 35, n. 6, p. 741-752, 2002.

CHACON-MIKAHIL, M.P.T. Estudo da variabilidade da freqüência cardíaca nos domínios do tempo e da freqüência antes e após o treinamento físico aeróbio em homens de meia-idade. 1998. 196p. Tese (Doutorado em Ciências) - Instituto de Biologia, Universidade Estadual de Campinas, Campinas.

CHACON-MIKAHIL, M.P.T.; FORTI, V.A.M.; CATAI, A.M.; SZRAJER, J.S.; GOLFETTI, R.; MARTINS, L.E.B.; LIMA-FILHO, E.C.; WANDERLEY, J.S.; MARINNETO, J.A.; MACIEL, B.C.; GALLO Jr, L. Cardiorespiratory adaptations induced by aerobic training in middle-aged men: the importance of a decrease in sympathetic stimulation for the contribution of dynamic exercise tachycardia. Braz $\mathbf{J}$ Med Biol Res, v. 31, n. 5, p. 705-712, 1998.

CHRIST, M.; SEYFFART, K; WEHLING, M. Attenuation of heart rate variability in postmenopausal women on progestin-containing hormone replacement therapy. Lancet, v. 353, n. 9168, p. 1939-1940, 1999.

CHRISTENSEN, N.J.; GALBO, H. Sympathetic nervous activity during exercise. Annu Rev Physiol, v. 45, p. 139-153, 1983.

COWAN, M.M.; GREGORY, L.W. Responses of pre and post-menopausal females to aerobic conditioning. Med Sci Sports Exerc, v. 17, n. 1, p. 138-143, 1985. 
COYLE, E.F.; MARTIN, W.H.; EHSANI, A.A.; HAGBERG, J.M.; BLOOMFIELD, S.A.; SINACORE, D.R.; HOLLOSZY, J.O. Blood lactate threshold in some welltrained ischemic heart disease patients. J Appl Physiol, v. 54, n. 1, p. 18-23, 1983.

CRIPPS, T.R.; MALIK, M.; FARRELL, T.G.; CAMM, A.J. Prognostic value of reduced heart rate variability after myocardial infarction: clinical evaluation of a new analysis method. Br Heart J, v. 65, n. 1, p. 14-19, 1991.

DAVY, K.P.; MINICLIER, N.L.; TAYLOR, J.A.; STEVENSON, E.T.; SEALS, D.R. Elevated heart rate variability in physically active postmenopausal women: a cardioprotective effect? Am J Physiol, v. 271, n. 2 Pt 2, p. H455-H460, 1996.

DAVY, K.P.; De SOUZA, C.A.; JONES, P.P.; SEALS, D.R. Elevated heart rate variability in physically active young and older adult women. Clin Sci (Lond), v. 94, n. 6 , p. 579-584, 1998.

De MEERSMAN, R.E. Heart rate variability and aerobic fitness. Am Heart J, v. 125, n. 3, p. 726-731, 1993.

De SOUZA, M.J.; MAGUIRE, M.S.; RUBIN, K.R.; MARESH, C.M. Effects of menstrual phase and amenorrhea on exercise performance in runners. Med Sci Sports Exerc, v. 22, n. 5, p. 575-580, 1990.

ESPELAND, M.A. Cardioprotective effects of postmenopausal hormone treatment. Am J Cardiol, v. 82, n. 10A, p. 37S-39S, 1998.

FARAG, N.H.; NELESEN, R.A.; PARRY, B.L.; LOREDO, J.S.; DIMSDALE, J.E.; MILLS, P.J. Autonomic and cardiovascular function in postmenopausal women: the effects of estrogen versus combination therapy. Am J Obstet Gynecol, v. 186, n. 5, p. 954-961, 2002.

FAZAN Jr., R.; BALLEJO, G.; SALGADO, M.C.O.; MORAES, M.F.D.; SALGADO, H.C. Heart rate variability and baroreceptor function in chronic diabetic rats. Hypertension, v. 30, n. 3, p. 632-635, 1997.

FERREIRA, V.; RIBEIRO, T.F.; AZEVEDO, G.D.; MILAN, L.A.; CATAI, A.M.; OLIVEIRA, L.; CRESCÊNCIO, J.C.; KOZUKI, R.T.; ARDUÍNE, A.C.R.; GALLO Jr, L.; SILVA DE SÁ, M.F.; SILVA, E. Determination of anaerobic threshold in Young and in postmenopausal women receiving or not hormonal replacement treatment. In: In: $13^{\text {TH }}$ ANNUAL MEETING OF THE NORTH AMERICAN MENOPAUSE SOCIETY (NAMS), 2002, Chicago. Anais..., Chicago, 2002. p. 92-92.

FLEG, J.L. Alterations in cardiovascular structure and function with advancing age. Am J Cardiol, v. 57, n. 5, p. 33C-44C, 1986. 
FORTI, V.A.M. Influência do treinamento físico aeróbio sobre as respostas cardiovasculares e respiratórias em mulheres na menopausa com e sem terapia de reposição hormonal. 1999. 213p. Tese (Doutorado em Educação Física) - Universidade Estadual de Campinas, Campinas.

FOSTER, C.; FITZGERALD, D.J.; SPATZ, P. Stability of the blood lactate-heart rate relationship in competitive athletes. Med Sci Sports Exerc, v. 31, n. 4, p. 578-582, 1999.

GALLO Jr, L.; MACIEL, B.C.; MARIN-NETO, J.A.; MARTINS, L.E.B.; LIMA-FILHO, E.C.; MANÇO, J.C. The use of isometric exercise as a means of evaluating the parasympathetic contribution to the tachycardia induced by dynamic exercise in normal man. Pflügers Arch, v. 412, p. 128-132, 1988.

GALLO Jr, L.; MACIEL, B.C.; MARIN-NETO, J.A.; MARTINS, L.E.B. Sympathetic and parasympathetic changes in heart rate control during dynamic exercise induced by endurance training in man. Braz J Med Biol Res, v. 22, p. 631-643, 1989.

GALLO Jr, L.; MACIEL, B.C.; MARIN-NETO, J.A.; MARTINS, L.E.B. Ajustes cardiovasculares ao exercício físico. Efeitos do treinamento aeróbio. Medicina, $v$. 23, n. 2, p. 101-106, 1990.

GALLO Jr, L.; MACIEL, B.C.; MARIN-NETO, J.A.; MARTINS, L.E.B.; LIMA-FILHO, E.C.; GOLFETTI, R.; CHACON, M.P.T.; FORTI, V.A.M. Control of heart rate during exercise in health and disease. Braz J Med Biol Res, v. 28, n. 11-12, p. 1179-1184, 1995.

GALLO Jr, L.; MACIEL, B.C.; MARTINS, L.E.B.; LIMA FILHO, E.C.; GOLFETTI, R. Quando o coração bate mais forte. Ciência Hoje, v. 21, p. 40-47, 1996.

GENEZZANI, A.R. Controversial issues in climacteric medicine (I) Cardiovascular disease and hormone replacement therapy. International Menopause Society Expert Workshop, 13-16 October 2000, Royal Society of Medicine, London, UK. Maturitas, v. 38, n. 3, p. 263-271, 2001.

GENSINI, G.F.; MICHELI, S.; PRISCO, D.; ABBATE, R. Menopause and risk of cardiovascular disease. Thromb Res, v. 84, n. 1, p. 1-19, 1996.

GOLDSMITH, R.L.; BIGGER Jr, J.T.; STEINMAN, R.C.; FLEISS, J.L. Comparison of 24-hour parasympathetic activity in endurance-trained and untrained young men. $\mathbf{J}$ Am Coll Cardiol, v. 20, n. 3, p. 552-558, 1992.

GOUVÊA, E.C.; CATAI, A.M.; SILVA, E; OLIVEIRA, L.; MILAN, L.A.; GALLO Jr., L. BARCELOS, S.R.; TREVELIN, L.C. Implementação e incorporação dos módulos de análise de dados e emissão de relatórios ao sistema computacional de eletrocardiografia de esforço. In: VI CONGRESSO DE INICIAÇÃO CIENTÍFICA DA UFSCar (CIC-UFSCar), 1998, São Carlos. Anais..., São Carlos, UFSCar, 1998. 
GRADY, D.; RUBIN, S.M.; PETITTI, D.B.; FOX, C.S.; BLACK, D.; ETTINGER, B.; ERNSTER, V.L.; CUMMINGS, S.R. Hormone therapy to prevent disease and prolong life in postmenopausal women. Ann Intern Med, v. 117, n. 12, p. 10161037, 1992.

GROSSMAN, P.; KAREMAKER, J.; WIELING, W. Prediction of tonic parasympathetic cardiac control using respiratory sinus arrhythmia: the need for respiratory control. Psychophysiology, v. 28, n. 2, p. 201-216, 1991.

HALBE, H.W. Tratado de ginecologia. $2^{a}$ ed. SP: Ed. Roca Ltda, 1998. v. 1.

HUIKURI, H.V.; PIKKUJAMSA, S.M.; AIRAKSINEN, K.E.; IKAHEIMO, M.J.; RANTALA, A.O.; KAUMA, H.; LILJA, M.; KESANIEMI, Y.A. Sex-related differences in autonomic modulation of heart rate in middle-aged subjects. Circulation, v. 94, n. 2, p. 122-125, 1996.

JURKOWSKI, J.E.; JONES, N.L.; TOEWS, C.J.; SUTTON, J.R. Effects of menstrual cycle on blood lactate, $\mathrm{O}_{2}$ delivery, and performance during exercise. J Appl Physiol, v. 51, n. 6, p. 1493-99, 1981.

KATONA, P.G.; McLEAN, M.; DIGHTON, D.H.; GUZ, A. Sympathetic and parasympathetic cardiac control in athletes and nonathletes at rest. J Appl Physiol, v. 52 , n. 6 , p. $1652-1657,1982$.

KENEMANS, P.; van UNNIK, G.A.; MIJATOVIC, V.; van der MOOREN, M.J. Perspectives in hormone replacement therapy. Maturitas, v. 38, p. S41-S48, 2001. (Suppl 1).

KLEIGER, R.E.; MILLER, J.P.; BIGGER Jr., J.T.; MOSS, A.J. Decreased heart rate variability and its association with increased mortality after myocardial infarction. Am J Cardiol, v. 59, n. 4, p. 256-262, 1987.

LINDQVIST, A. Noninvasive methods to study autonomic nervous control of circulation. Acta Physiol Scand, v. 588, p. 1-107, 1990. (Suppl).

LOIMAALA, A.; HUIKURI, H.; OJA, P.; PASANEN, M.; VUORI, I. Controlled 5-mo aerobic training improves heart rate but not heart rate variability or baroreflex sensitivity. J Appl Physiol, v. 89, n. 5, p. 1825-1829, 2000.

LONGO, A.; FERREIRA, D.; CORREIA, M.J. Variabilidade da freqüência cardíaca. Rev Port Cardiol, v. 14, n. 3, p. 241-262, 1995.

MACIEL, B.C.; GALLO Jr., L.; MARIN-NETO, J.A.; TERRA-FILHO, J.; MANÇO, J.C. Efficacy of pharmacological blockade of the cardiac parasympathetic system with atropine in normal men. Braz J Med Biol Res, v. 18, n. 3, p. 303-308, 1985. 
MACIEL, B.C.; GALLO Jr., L.; MARIN-NETO, J.A.; MARTINS, L.E.B. Autonomic nervous control of the heart rate during dynamic exercise in normal men. Clin Sci (Lond), v. 71, n. 4, p. 457-460, 1986.

MALPAS, S.C.; MALING, T.J. Heart-rate variability and cardiac autonomic function in diabetes. Diabetes, v. 39, n. 10, p. 1177-1181, 1990.

MARÃES, V.R.F.S. Estudo da variabilidade da freqüência cardíaca durante exercício físico dinâmico em voluntários sadios. 1999. 225p. Tese (Mestrado em Biologia Funcional e Molecular) - Instituto de Biologia, Universidade Estadual de Campinas, Campinas.

MARÃES, V. R. F. S.; SILVA, E.; CATAI, A. M.; OLIVEIRA, L.; MOURA, M.S.A.; TREVELIN, L.C.; GALLO Jr., L. Study of variability of instantaneous heart rate to the detection of anaerobic threshold. In: L CONGRESSO NAZIONALE DELLA SOCIETA ITALIANA DI ANATOMIA, 1996, Catania. Resumos..., Roma: Archivio Italiano di Anatomia ed Embriologia, 1996, v.101, p. 123-123.

MARÃES, V. R. F. S.; RIBEIRO, T. F.; CATAI, A. M.; OLIVEIRA, L.; GALLO Jr., L.; SILVA, E. The heart rate variability in the characterization of the sympathetic and parasympathetic nervous system of the heart. In: 7TH WORLD CONGRESS ON HEART FAILURE - MECHANISMS AND MANAGEMENT, 2000a, Vancouver. Resumos..., Vancouver: The Journal of Heart Failure, 2000a, v.6, p.113-113.

MARÃES, V.R.F.S.; SILVA, E.; CATAI, A.M.; GALLO Jr., L.; OLIVEIRA, L.; RIBEIRO, T.F.; MOURA, M.S.A.; CANOTILHO, M.M. Avaliação da capacidade física de pacientes com doença pulmonar obstrutiva crônica de grau leve e moderado a partir da resposta da freqüência cardíaca: relato de caso. Rev Soc Cardiol Estado de São Paulo, v.10, n.3, p. 6-15, 2000b. (Suppl A).

MARÃES, V. R. F. S.; SILVA, E.; CATAI, A. M.; RIBEIRO, T. F.; GALLO Jr., L. The heart rate variability in dynamic exercise. Its possible role to signal anaerobic threshold. In: 2000 APS INTERSOCIETY MEETING: THE INTEGRATIVE BIOLOGY OF EXERCISE, 2000c, Portland. Resumos..., Portland: The Physiologist, 2000c, v. 43 , n. 4 , p. 339-339.

MASSIN, M.M.; MAEYNS, K.; WITHOFS, N.; RAVET, F.; GÉRARD, P. Circadian rhythm of heart rate and heart rate variability. Arch Dis Child, v. 83, n. 2, p. 179182, 2000.

McARDLE, W.D.; KATCH, F.I.; KATCH, V.L. Regulação e integração cardiovasculares In: McARDLE, W.D.; KATCH, F.I.; KATCH, V.L. Fisiologia do exercício: energia, nutrição e desempenho humano. $4^{\mathrm{a}}$ ed., RJ: Guanabara Koogan, 1998. Cap. 16, p. 271-281.

McCABE, P.M.; PORGES, S.W.; CAITER, C.S. Heart period variability during estrogen exposure and withdrawal in female rats. Physiol Behav, v. 26, n. 3, p. 535-538, 1981. 
MENDELSOHN, M.E. Mechanisms of estrogen action in the cardiovascular system. J Steroid Biochem Mol Biol, v. 74, n. 5, p. 337-343, 2000.

MERCURO, G.; PODDA, A.; PITZALIS, L.; ZONCU, S.; MASCIA, M.; MELIS, G.B.; ROSANO, G.M.C. Evidence of a role of endogenous estrogen in the modulation of autonomic nervous system. Am J Cardiol, v. 85, n. 6, p. 787-789, 2000.

MIDDLEKAUFF, H.R.; NGUYEN, A.H.; NEGRÃO, C.E.; NITZSCHE, E.U.; HOH, C.K.; NATTERSON, B.A.; HAMILTON, M.A.; FONAROW, G.C.; HAGE, A.; MORIGUCHI, J.D. Impact of acute mental stress on sympathetic nerve activity and regional blood flow in advanced heart failure: implications for "triggering" adverse cardiac events. Circulation, v. 96, n. 6, p. 1835-1842, 1997.

MIGLIARO, E.R.; CONTRERAS, P.; BECH, S.; ETXAGIBEL, A.; CASTRO, M.; RICCA, R.; VICENTE, K. Relative influence of age, resting heart rate and sedentary life stile in short-term analysis of heart rate variability. Braz $\mathbf{J}$ Med Biol Res, v. 34, n. 4, p. 493-500, 2001.

MITCHELL, J.H. Neural control of the circulation during exercise. Med Sci Sports Exerc, v. 22, n. 2, p. 141-154, 1990.

MUKAI, S.; HAYANO, J. Heart rate and blood pressure variabilities during graded head-up tilt. J Appl Physiol, v. 78, n. 1, p. 212-216, 1995.

NISKANEN, L.; LAITINEN, T.; TUPPURAINEN, M.; SAARIKOSKI, S.; KRÖGER, H.; ALHAVA, E.; HARTIKAINEN, J. Does postmenopausal hormone replacement therapy affect cardiac autonomic regulation in osteoporotic women? Menopause, v. 9, n. 1, p. 52-57, 2002.

NÓBREGA, A.C.L.; ARAÚJO, C.G.S. Heart rate transient at the onset of active and passive dynamic exercise. Med Sci Sports Exerc, v. 25, n. 1, p. 37-41, 1993.

NORDENFELT, I. Hemodynamic response to exercise after combined sympathetic and parasympathetic blockade of the heart. Cardiovasc Res, v. 5, n. 2, p. 215-222, 1971.

NOTELOVITZ, M.; FIELDS, C.; CARAMELLI, K.; DOUGHERTY, M.; SCHWARTZ, A.L. Cardiorespiratory fitness evaluation in climacteric women: comparison of two methods. Am J Obstet Gynecol, v. 154, n.5, p. 1009-1013, 1986.

OLIVEIRA, L. MILAN, L.A.; CATAI, A.M.; GALLO Jr, L.; SILVA, E. Modelo semiparamétrico de análise do comportamento da freqüência cardíaca. In: XVII REUNIÃO ANUAL DA FEDERAÇÃO DE SOCIEDADES DE BIOLOGIA EXPERIMENTAL - FeSBE, 2002, Salvador. CD Rom..., Salvador, 2002.

PIKKUJÂMSÂ, S.M.; MÂKIKALLIO, T.H.; AIRAKSINEN, K.E.J.; HUIKURI, H.V. Determinants and interindividual variation of $R-R$ interval dynamics in healthy middle-aged subjects. Am J Physiol, v. 280, n. 3, p. H1400-H1406, 2001. 
PINES, A.; MIJATOVIC, V.; van der MOOREN, M.J.; KENEMANS, P. Hormone replacement therapy and cardioprotection: basic concepts and clinical considerations. Eur J Obstet Gynecol Reprod Biol, v. 71, n. 2, p. 193-197, 1997.

RIBEIRO, T.F.; GOMES, V.R.; MOURA, M.S.A.; MARÃES, V.R.F.S.; CATAI, A.M.; OLIVEIRA, L.; TREVELIN, L.C.; GALLO Jr., L.; SILVA, E. Estudo do limiar de anaerobiose em mulheres sedentárias durante esforço físico dinâmico. In: XIII REUNIÃO ANUAL DA FEDERAÇÃO DE SOCIEDADES DE BIOLOGIA EXPERIMENTAL - FeSBE, 1998, Caxambu. Anais..., Caxambu, 1998. p. 258-258.

RIBEIRO, T. F.; CUNHA, A.; LOURENÇO, G. C. D.; MARÃES, V. R. F. S.; CATAI, A. M.; GALLO Jr., L.; SILVA, E. Estudo da variabilidade da freqüência cardíaca (VFC) a partir dos intervalos R-R de voluntários de meia idade, saudável e coronariopata: relato de caso. Rev Soc Cardiol Estado de São Paulo, v.10, n.1, p.1-10, 2000. (Suppl A).

RIBEIRO, T. F.; AZEVEDO, G. D.; CRESCÊNCIO, J. C.; MARÃES, V. R. F. S.; PAPA, V.; CATAI, A. M.; VERZOLA, R. M. M.; OLIVEIRA, L.; SILVA de SÁ, M. F.; GALLO Jr., L.; SILVA, E. Heart rate variability under resting conditions in postmenopausal and young women. Braz J Med Biol Res, v. 34, n. 7, p. 871-877, 2001a.

RIBEIRO, T.F.; AZEVEDO, G.D.; MARÃES, V.R.F.S.; CATAI, A.M.; CRESCÊNCIO, J.C.; OLIVEIRA, L.; GALLO Jr.; L.; FERRIANI, R.A.; SILVA DE SÁ, M.F.; SILVA, E. Determination of aerobic capacity in postmenopausal and young women by analysis of heart rate variability. Organizado por GENEZZANI, A.R.; ARTINI, P.G.; PETRAGLIA, F. Recent Research in Gynecological Endocrinology, Londres, UK, v. 1, p. 177-180, 2001b.

RIBEIRO, T.F. Avaliação da modulação autonômica da atividade eferente do coração pela variabilidade da freqüência cardíaca em mulheres sedentárias em repouso e em exercício físico. 2001. 106 p. Tese (Mestrado em Fisioterapia) Universidade Federal de São Carlos, São Carlos.

ROBINSON, BF; EPSTEIN, S.E.; BEISER, G.D.; BRAUNWALD, E. Control of heart rate by the autonomic nervous system. Studies in man on the interrelation between baroreceptor mechanisms and exercise. Circ Res, v. 19, n. 2, p. 400-411, 1966.

ROSANO, G.M.C.; PATRIZI, R.; LEONARDO, F.; PONIKOWSKI, P.; COLLINS, P.; SARREL, P.M.; CHIERCHIA, S.L. Effect of estrogen replacement therapy on heart rate variability and heart rate in healthy postmenopausal women. Am $\mathbf{J}$ Cardiol, v. 80, n. 6, p. 815-817, 1997.

ROSÁRIO, J. L. P.; CATAI, A. M.; SILVA, E.; VERZOLA, R. M. M.; GALLO Jr., L.; OLIVEIRA, L.; MOURA, M. S. A. Heart rate variability in patients with coronary heart disease and arterial hypertension. In: 7TH WORLD CONGRESS ON HEART FAILURE - MECHANISMS AND MANAGEMENT, 2000, Vancouver. Resumos..., Vancouver: The Journal of Heart Failure, 2000, v. 6, p.113-113. 
ROWELL, L.B. Human circulation: regulation during physical stress. $1^{\text {a }}$ ed. New York: Oxford University Press, 1986.

SAKABE, D.I.; FERREIRA, V.; RIBEIRO, T.F.; AZEVEDO, G.D.; MILAN, L.A.; CATAI, A.M.; OLIVEIRA, L.; GALLO Jr, L.; SILVA DE SÁ, M.F.; SILVA, E. Use of a semi-parametric model for the analysis of heart rate variability (HRV) in order to determine the anaerobic threshold of middle-aged men and postmenopausal women receiving or not hormonal replacement therapy. In: $13^{\text {th }}$ Annual Meeting of The North American menopause Society (NAMS), 2002, Chicago. Anais..., Chicago, 2002. p. 94-94.

SANADA, M.; NAKAGAWA, H.; KODAMA, I.; SAKASITA, T.; OHAMA, K. The effect of hormone replacement therapy on metabolism of lipoprotein remnants in postmenopausal women. Maturitas, v. 34, n. 1, p. 75-82, 2000.

SATO, I.; HASEGAWA, Y.; HOTTA, K. Autonomic Nervous Control of the heart in exercising man. Pflügers Arch, v. 384, n. 1, p. 1-7, 1980.

SCHUIT, A.J.; van AMELSVOORT, L.G.P.M.; VERHEIJ, T.C.; RIJNEKE, R.D.; MAAN, A.C.; SWENNE, C.A.; SCHOUTEN, E.G. Exercise training and heart rate variability in older people. Med Sci Sports Exerc, v. 31, n. 6, p. 816-821, 1999.

SCHWARTZ, J.B.; GIBB, W.J.; TRAN, T. Aging effects on heart rate variation. J Gerontol, v. 46, n. 3, p. M99-M106, 1991.

SEALS, D.R.; CHASE, P.B.; Influence of physical training on heart rate variability and baroreflex circulatory control. J Appl Physiol, v. 66, n. 4, p. 1886-1895, 1989.

SEALS, D.R.; VICTOR, R.G. Regulation of muscle sympathetic nerve activity during exercise in humans. Exerc Sport Sci Rev, v. 19, p. 313-349, 1991.

SEALS, D.R.; TAYLOR, J.A.; NG, A.V.; ESLER, M.D. Exercise and aging: autonomic control of the circulation. Med Sci Sports Exerc, v. 26, n. 5, p. 568-576, 1994.

SHANNON, D.C.; CARLEY, D.W.; BENSON, H. Aging of modulation of heart rate. Am J Physiol, v. 253, n. 4 Pt 2, p. H874- H877, 1987.

SILVA, C.S.; MARQUES, L.S.; MORAES, F.R.; CATAI, A.M.; OLIVEIRA, L.; SILVA, $E$. Investigação da variabilidade da freqüência cardíaca de mulheres nos períodos manhã e noite. Rev Bras Fisioter, v. 5, n. 2, p. 65-71, 2001.

SILVA, E. Modelos matemáticos da resposta da freqüência cardíaca ao exercício físico dinâmico em indivíduos normais. 1988. 187p. Tese (Mestrado em Ciências Biológicas) - Instituto de Biologia, Universidade Estadual de Campinas, Campinas. 
SILVA, E.; CATAI, A.M.; TREVELIN, L.C.; GUIMARÃES, J.O.; SILVA Jr., L.P.; SILVA, L.M.P.; OLIVEIRA, L.; MILAN, L.A.; MARTINS, L.E.B.; GALLO Jr., L. Design of a computerized system to evaluate the cardiac function during dynamic exercise. In: WORLD CONGRESS ON MEDICAL PHYSICS AND BIOMEDICAL ENGINEERING, 1994, Rio de Janeiro. Annals..., Rio de Janeiro, 1994. p. 409-409.

SINGH, J.P.; LARSON, M.G.; TSUJI, H.; EVANS, J.C.; O'DONNELL, C.J.; LEVY, D. Reduced heart rate variability and new-onset hypertension: insights into pathogenesis of hypertension: the Framingham Heart Study. Hypertension, v. 32, n. 2, p. 293-297, 1998.

STEINGOLD, K.A.; LAUFER, L.; CHETKOWSKI, R.J.; De FAZIO, J.D.; MATT, D.W.; MELDRUM, D.R.; JUDD, H.L. Treatment of hot flashes with transdermal estradiol administration. J Clin Endocrinol Metab, v. 61, n. 4, p. 627-632, 1985.

SULLIVAN, J.M.; FOWLKES, L.P. The clinical aspects of estrogen and the cardiovascular system. Obstet Gynecol, v. 87, n. 2, p. 36S-43S, 1996. (Suppl)

TASKE FORCE of the European Society of Cardiology and the North American Society of Pacing and Electrophysiology. Heart rate variability: standards of measurement, physiological interpretation and clinical use. Circulation, v. 93, n. 5, p. 1043-1065, 1996.

TATE, C.A.; HYEK, M.F.; TAFFET, G.E. Mechanism for the responses of cardiac muscle to physical activity in old age. Med Sci Sports Exerc, v. 26, n. 5, p. 561$567,1994$.

TSUJI H.; VENDITTI Jr, F.J.; MANDERS, E.S.; EVANS, J.C.; LARSON, M.G.; FELDMAN, C.L.; LEVY, D.; Determinants of heart rate variability. $\mathbf{J}$ Am Coll Cardiol, v. 28, n. 6, p. 1539-1546, 1996.

TULPPO, M.P.; MÂKIKALLIO, T.H.; TAKALA, T.E.S.; SEPPÂNEN, T.; HUIKURI, H.V. Quantitative beat-to-beat analysis of heart rate dynamics during exercise. Am J Physiol, v. 271, n. 1 Pt 2, p. H244-H252, 1996.

TULPPO, M.P.; MÂKIKALLIO, T.H.; SEPPÂNEN, T.; LAUKKANEN, R.T.; HUIKURI, H.V. Vagal modulation of heart rate during exercise: effects of age and physical fitness. Am J Physiol, v. 274, n. 2 Pt 2, p. H424-H429, 1998.

VIRTANEN, I.; POLO, O.; POLO-KANTOLA, P.; KUUSELA, T.; EKHOLM, E. The effect of estrogen replacement therapy on cardiac autonomic regulation. Maturitas, v. 37 , n. 1 , p. $45-51,2000$.

WALSH, R.A. Cardiovascular effects of the aging process. Am J Med, v. 82, p. 3440, 1987. (Suppl 1B).

WASSERMAN, K. BEAVER, W.L.; WHIPP, B.J. Gas exchange theory and the lactic acidosis (anaerobic) threshold. Circulation, v. 81, n. 1, p. II14-II30, 1990. (Suppl II). 
WASSERMAN, K.; HANSEN, J.E.; SUE, D.; WHIPP, B.J.; CASABURI, R. Principles of exercise testing and interpretation. $3^{\mathrm{a}}$ ed. Philadelphia: Williams \& Wilkins, 1999.

WELTMAN, A.; SNEAD, D.; STEIN, P.; SEIP, R.; SCHURRER, R.; RUTT, R.; WELTMAN, J. Reliability and validity of a continuous incremental treadmill protocol for the determination of lactate threshold, fixed blood lactate concentrations and $\mathrm{VO}_{2}$ max. Int J Sports Med, v. 11, n. 1, p. 26-32, 1990.

WHITEHEAD, M.I.; HILLARD, T.C.; CROOK, D. The role and use of progestogens. Obstet Gynecol, v. 75, n. 4, p. 59S-76S, discussion 81S-83S, 1990. (Suppl).

WILD, R.A. Estrogen: effects on the cardiovascular tree. Obstet Gynecol, v. 87, n. 2, p. 27S-35S, 1996. (Suppl).

WILLIAMSON, J.W.; NÓBREGA, A.C.L.; WINCHESTER, P.K.; ZIM, S.; MITCHELL, J.H. Instantaneous heart rate increase with dynamic exercise: central command and muscle-heart reflex contributions. J Appl Physiol, v. 78, n. 4, p. 1273-1279, 1995.

YAMAMOTO, Y.; HUGHSON, R.L.; PETERSON, J.C. Autonomic control of heart rate during exercise studied by heart rate variability spectral analysis. J Appl Physiol, v. 71, n. 3, p. 1136-1142, 1991.

YAMASAKI, Y.; KODAMA, M.; MATSUHISA, M.; KISHIMOTO, M.; OZAKI, H.; TANI, A.; UEDA, N.; ISHIDA, Y.; KAMADA, T. Diurnal heart rate variability in healthy subjects: effects of aging and sex difference. Am J Physiol, v. 271, n. 1, p. H303H310, 1996. 


\section{APÊNDICES}

\section{Apêndice 1}

Incluímos neste apêndice:

- Parecer de aprovação do Comitê de Ética em Pesquisa da UFSCar;

- Parecer de aprovação do Comitê de Ética em Pesquisa do Hospital das Clínicas da Faculdade de Medicina de Ribeirão Preto da USP;

- Termo de Consentimento Formal - Pós-Informação, para pesquisas em seres humanos 


\section{Aprovação do Comitê de Ética em Pesquisa da UFSCar}

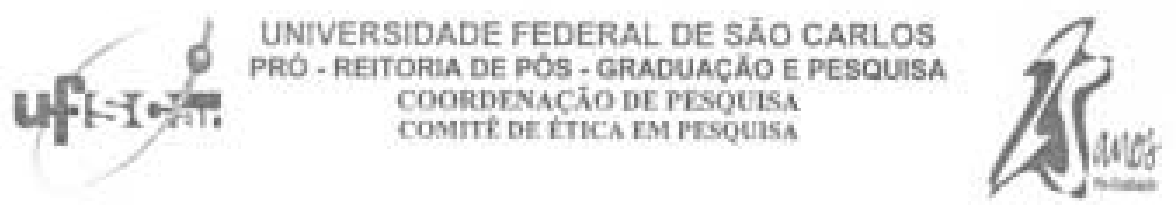

0 Cosiot de Éliks em Pesyuisa (CHP) da UFSCar, registrado no CONEP/Cunselho Nacional de Saúle, pelo ato de 18 de março de 1997. DELIBEROU aprovaçbo sem restriçles a projeto crim protocalo a" 019 e título: "A INVLUENCIA DA IDADE E DA REPOSIÇO HOIRMONAL. SOHRE A MODULAÇÃO AUTONÓMICA DO CORAÇ̃o E NO L.IMIAR DE ANAROBIOSE" com oñentaço do Profa. Dra. Esier da Sitiva.

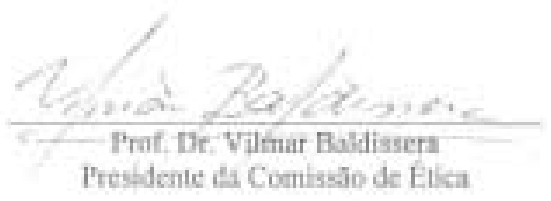




\section{Aprovação do Comitê de Ética em Pesquisa do Hospital das Clínicas da Faculdade de Medicina de Ribeirão Preto da USP}

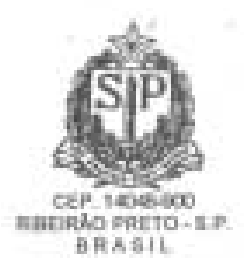

Oficio $\mathrm{n}^{\circ} 3527 / 2001$

CEP/SPC

\author{
HOSPITAL DAS CLINICAS DA FACULDADE DE MEDICINA \\ DE RIBEIRAO PRETO DA UNIVERSIDADE DE SAOO PAULO

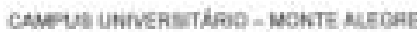

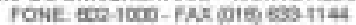

Ribeirầo Preto, 14 de dezembro de 2001

Prezada Benhora:

O trabalho intitulado "A INFLUÊNCIA DA

IDADE E DA REPOSIÇĀO HORMONAL SOBRE A MODULAÇĀo AUTONŌMICA DO CORAÇÃo E O LIMIAR DE ANAEROBIOSE", foi analisado pelo Comité de Etica em Pesquisa, em sua 121* Reuniâo Ordinária realizada em 03/12/2001, e enquadrado na categoria: APROVADO, de acordo com o Processo HCRP n* 7966/2001.

Aproveito a oportunidade para apresentar a Vossa Senhoria protestos de estima c consideraçăo.

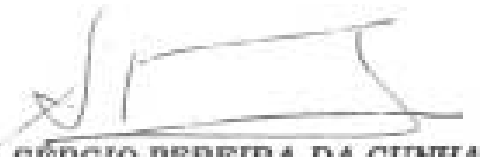

PROF. DR. SERGIO PEREIRA DA CUNHA

Coordenador do Comitể de Êtiea em Pesquina do HCFMrP-USP

Ilustrissima Senhora

VALÉRIA FERREIRA-A/C PHOF-DH. LOUHEQ⿻日, GALIO JONIOR

Depto. de Clinica Médica - Divisắo de Cardiologia

Eัm mâิoง 


\section{Termo de Consentimento Formal}

\section{Universidade de São Paulo \\ Hospital das Clínicas da Faculdade de Medicina de Ribeirão Preto}

\section{Termo de Consentimento Formal - Pós-Informação}

Pesquisa: A influência da idade e da reposição hormonal sobre a modulação autonômica do coração e o limiar de anaerobiose

Pesquisadores Responsáveis: Prof. Dr. Lourenço Gallo Júnior

Profa. Dra. Ester da Silva

Valéria Ferreira

A pesquisa tem por objetivo avaliar como os nervos que controlam o seu coração respondem frente a um exercício físico e medir a intensidade de esforço que seu organismo pode realizar sem trazer riscos para o seu coração, quando a senhora estiver fazendo os exercícios da sua vida diária (andar, serviço de casa,...).

Desde que a senhora concorde será incluída em nosso estudo e submetida a uma avaliação clínica com medida de pressão, pulso, peso, altura, etc., além de alguns exames tais como: exame de sangue, dosagem de açúcar e da gordura do sangue, exames das substâncias eliminadas pelos rins, que servirão para avaliar seu estado de saúde. 
Os exames que realizaremos neste estudo são testes de esforço em bicicleta de laboratório, fixa no chão, que permitem que cada pessoa pedale em intensidades diferentes. Antes de realiza-lo a senhora fará também um exame em repouso e outro em esforço para ver se está tudo bem com o funcionando do coração e do pulmão (eletrocardiograma e teste de esforço máximo na esteira). Caso a senhora apresente a avaliação normal, será levada ao Laboratório de Fisiologia do Exercício, para conhecer os equipamentos e para receber maiores esclarecimentos sobre os testes que fará. Todos estes exames serão feitos sem que seja necessário usar agulhas para perfurar veias ou qualquer outra parte do corpo. Estes exames não oferecem riscos de complicações para sua saúde, e os desconfortos que poderão ocorrer com os testes de esforço na bicicleta são só um pouco de cansaço muscular e falta de ar, à medida que aumenta a intensidade do esforço, como acontece quando a senhora faz exercícios mais intensos nos trabalhos de casa (lavar roupa, varrer a casa, etc).

Os benefícios que terá com os exames realizados incluem a avaliação de possíveis alterações do funcionamento do coração e pulmão que ocorram nos exames clínicos, laboratoriais e de esforço. Caso se encontre alguma anormalidade nos exames, a senhora será encaminhada ao setor do Hospital das Clínicas da Faculdade de Medicina de Ribeirão Preto, para orientação específica. Os testes vão medir a sua capacidade para fazer exercícios físicos, no trabalho ou em programas de exercícios usados para diminuir os efeitos do envelhecimento, da parada das regras, e na prevenção das doenças do coração. 
$\mathrm{Eu}$, portadora do $R G n^{\circ}$. , residente a Rua:

$\mathrm{n}^{\circ}$.

Bairro: , Cidade: Estado:

tendo recebido as informações acima e ciente dos meus direitos abaixo relacionados, voluntariamente concordo em participar de um programa científico nos termos do projeto de pesquisa proposto pelo Laboratório de Fisiologia do exercício do Setor de Cardiologia do Hospital das Clínicas da Faculdade de Medicina de Ribeirão Preto - USP.

1. A garantia de receber resposta a qualquer pergunta ou esclarecimento a qualquer dúvida a cerca dos procedimentos, riscos e benefícios e outros relacionados com a pesquisa;

2. A liberdade de retirar meu consentimento a qualquer momento e deixar de participar no estudo sem que isso traga prejuízo à continuação do meu cuidado e tratamento;

3. A segurança de que não serei identificado e que será mantido o caráter confidencial da informação relacionada com minha privacidade;

4. O compromisso de me proporcionar informação atualizada durante o estudo, ainda que esta possa afetar minha vontade de continuar participando.

5. A disponibilidade de tratamento médico por parte da Instituição à Saúde, em caso de danos que justifiquem, diretamente causados pela pesquisa.

Ribeirão Preto, de de 200 


\section{Apêndice 2}

Neste apêndice estão apresentados as tabelas de:

- Resultado dos exames laboratoriais;

- Resultado do teste de esforço físico dinâmico contínuo de avaliação cardiológica;

- Tipo de medicação hormonal utilizada pelo grupo de voluntárias PMCRH. 
TABELA 3. Resultado dos exames laboratoriais de hemograma; urina tipo 1; glicemia, uréia e creatinina, em $\mathrm{mg} / \mathrm{dl}$; triglicérides, colesterol total, LDL, HDL, em $\mathrm{mg} / 100 \mathrm{ml}$ e TSH, em $\mathrm{mUl} / \mathrm{ml}$ das voluntárias jovens, pós-menopausa sem reposição hormonal (PMSRH) e pós-menopausa com reposição hormonal (PMCRH).

\begin{tabular}{|c|c|c|c|c|}
\hline \multirow{3}{*}{ Exames } & \multirow{3}{*}{$\begin{array}{l}\text { Valores de } \\
\text { Referência* }^{*}\end{array}$} & \multicolumn{3}{|c|}{ Valores Observados } \\
\hline & & $\begin{array}{l}\text { Jovens } \\
(n=10)\end{array}$ & $\begin{array}{l}\text { PMSRH } \\
(n=13)\end{array}$ & $\begin{array}{l}\text { PMCRH } \\
(n=9)\end{array}$ \\
\hline & & $X \pm D P$ & $X \pm D P$ & $X \pm D P$ \\
\hline Hemograma & Normal & Normal & Normal & Normal \\
\hline Urina tipo 1 & Normal & Normal & Normal & Normal \\
\hline Glicemia (mg/dl) & 76 a 120 & $79,9 \pm 6,21$ & $85,5 \pm 9,41$ & $94,0 \pm 6,77$ \\
\hline Uréia (mg/dl) & 16 a 46 & $33,1 \pm 3,65$ & $29,5 \pm 5,58$ & $32,5 \pm 4,48$ \\
\hline Creatinina (mg/dl) & 0,7 a 1,3 & $1,0 \pm 0,07$ & $0,9 \pm 0,20$ & $1,0 \pm 0,10$ \\
\hline $\begin{array}{l}\text { Triglicérides } \\
(\mathrm{mg} / 100 \mathrm{ml})\end{array}$ & $<200$ & $86 \pm 28,11$ & $132 \pm 53,17$ & $163 \pm 97,95$ \\
\hline $\begin{array}{l}\text { Colesterol total } \\
(\mathrm{mg} / 100 \mathrm{ml})\end{array}$ & $<200$ & $167 \pm 25,67$ & $218 \pm 46,64$ & $220 \pm 68,02$ \\
\hline LDL (mg/100ml) & $<130$ & $91 \pm 19,78$ & $147 \pm 43,68$ & $128 \pm 53,34$ \\
\hline HDL (mg/100ml) & $>35$ & $57 \pm 9,26$ & $44 \pm 10,07$ & $59 \pm 17,91$ \\
\hline TSH (mUl/ml) & 0,3 a 4,0 & $2,0 \pm 0,83$ & $1,9 \pm 2,04$ & $1,5 \pm 1,67$ \\
\hline $\begin{array}{l}\mathrm{X}=\text { média; } \mathrm{DP}=\mathrm{d} \\
\text { lipoproteína de alta } \\
\text { miligrama por decili } \\
\text { sangue; } \mathrm{mUl} / \mathrm{ml}= \\
\text { Laboratório Médico }\end{array}$ & $\begin{array}{l}\text { padrão; LDL } \\
\text { idade; TSH = } \\
\text { e sangue; m } \\
\text { unidades int } \\
\text { aricondi. }\end{array}$ & $\begin{array}{l}\text { lipoproteína } \\
\text { lormônio esti } \\
100 \mathrm{ml}=\text { mil } \\
\text { lacionais por }\end{array}$ & $\begin{array}{l}\text { de baixa dens } \\
\text { fulante da tire } \\
\text { yrama por } 10 \\
\text { mililitro de sa }\end{array}$ & $\begin{array}{l}\text { dade; } \mathrm{HDL}= \\
\text { óide; } \mathrm{mg} / \mathrm{dl}= \\
\text { mililitros de } \\
\text { ggue. }{ }^{*} \text { Fonte }\end{array}$ \\
\hline
\end{tabular}


TABELA 4. Velocidade, em mph, e porcentagem (\%) de inclinação da esteira, valores de freqüência cardíaca (FC), em bpm, pressão artéria sistólica (PAS) e diastólica (PAD), em $\mathrm{mmHg}$, atingidos no pico do teste de exercício físico dinâmico contínuo de avaliação cardiológica e valores de freqüência cardíaca máxima prevista para a idade (FC máx prev), em bpm, das voluntárias jovens, pós-menopausa sem reposição hormonal (PMSRH) e pós-menopausa com reposição hormonal $(\mathrm{PMCRH})^{*}$.

\begin{tabular}{|c|c|c|c|}
\hline Variáveis & $\begin{array}{c}\text { Jovens }(n=10) \\
X \pm D P\end{array}$ & $\begin{array}{c}\text { PMSRH }(n=13) \\
X \pm D P\end{array}$ & $\begin{array}{c}\text { PMCRH }(n=9) \\
X \pm D P\end{array}$ \\
\hline Velocidade (mph) & $4,7 \pm 0,50$ & $3,4 \pm 0,80$ & $3,5 \pm 0,92$ \\
\hline \% de inclinação & $17 \pm 1,40$ & $14 \pm 1,91$ & $14 \pm 2,19$ \\
\hline FC (bpm) & $182 \pm 11,89$ & $149 \pm 18,52$ & $163 \pm 16,20$ \\
\hline PAS (mmHg) & $138 \pm 18,14$ & $171 \pm 33,53$ & $166 \pm 17,40$ \\
\hline PAD (mmHg) & $74 \pm 8,43$ & $87 \pm 9,24$ & $91 \pm 10,54$ \\
\hline FC máx prev (bpm) & $186 \pm 2,87$ & $153 \pm 5,28$ & $155 \pm 5,40$ \\
\hline
\end{tabular}


TABELA 5. Tipo de medicação hormonal utilizada pelas voluntárias pós-menopausa com reposição hormonal $(n=9)$.

\begin{tabular}{cccc}
\hline Voluntárias & Medicamento & Princípio ativo & $\begin{array}{c}\text { Dosagem } \\
(\mathbf{m g})\end{array}$ \\
\hline DMP & Premarim & Estrogênios conjugados & 0,625 \\
ES & Cycrim & Acetato de medroxiprogesterona & 2,5 \\
ESV & Premarin & Estrogênios conjugados & 0,625 \\
IPZ & Premarin & Estrogênios conjugados & 0,625 \\
MCGP & Premarin & Estrogênios conjugados & 0,625 \\
& Livial & Tibolona & 2,5 \\
NARS & Premarim & Estrogênios conjugados & 0,625 \\
& Cycrim & Acetato de medroxiprogesterona & 2,5 \\
OSA & Premarin & Estrogênios conjugados & 0,625 \\
& Premarim & Estrogênios conjugados & 0,625 \\
& Cycrim & Acetato de medroxiprogesterona & 2,5 \\
& Premarin & Estrogênios conjugados & 0,625 \\
\hline
\end{tabular}




\section{Apêndice 3}

Apresentamos neste apêndice:

- Descrição técnica do modelo matemático e estatístico semiparamétrico, adaptado para determinação do limiar de anaerobiose pela resposta da freqüência cardíaca. 


\section{Modelo matemático e estatístico semiparamétrico}

Este modelo é composto por componentes paramétricos e não paramétricos em relação à mesma variável e propõe a separação desses componentes da série em questão, no caso os dados de FC obtidas no intervalo selecionado do TEFDD-D, em duas partes. A primeira parte apresenta elementos com tendências lineares em relação ao modelo ajustado, com configuração paramétrica correspondente aos resíduos da série. A segunda parte contém os elementos da primeira parte com configuração não paramétrica. A análise da tendência de inclinação dos valores de FC é dada por uma análise de variância e teste $t$ de Student com nível de significância de $\alpha=0,05$. $O$ componente paramétrico admite que os resíduos da série têm distribuição normal e, aplicando-se o teste de DurbinWatson, verifica-se autocorrelação significante entre os resíduos. O componente não paramétrico se ajusta aos componentes cíclicos da série e para isso é preciso uma ortogonalização em relação aos elementos do componente paramétrico, de um trecho selecionado (120 a 300 s), onde se espera observar uma maior estabilidade da série. O modelo analisa os dados e apresenta resultados respectivos a tendência de inclinação, se positiva ou negativa, ou seja, o quanto esta inclinação difere de zero, com $p<0,05$ (OLIVEIRA et al., 2002). 